Published in final edited form as:

Cochrane Database Syst Rev. ; (4): CD003794. doi:10.1002/14651858.CD003794.pub3.

\title{
Combined corticosteroid and long-acting beta-agonist in one inhaler versus placebo for chronic obstructive pulmonary disease
}

\author{
Luis Javier Nannini ${ }^{1}$, Christopher J Cates ${ }^{2}$, Toby J Lasserson ${ }^{3}$, and Phillippa Poole ${ }^{4}$ \\ ${ }^{1}$ Pulmonary Section, Hospital E Peron, G. Baigorria, Argentina ${ }^{2}$ Community Health Sciences, St \\ George's, University of London, London, UK ${ }^{3}$ Cochrane Airways Group, Divison of Community \\ Health Sciences, St George's University of London, London, UK ${ }^{4}$ University of Auckland, \\ Auckland, New Zealand
}

\begin{abstract}
Background-Long-acting beta-agonists and inhaled corticosteroids have both been recommended in guidelines for the treatment of chronic obstructive pulmonary disease. Their coadministration in a combined inhaler may facilitate adherence to medication regimens, and improve efficacy.

Objectives-To assess the efficacy of combined inhaled corticosteroid and long-acting betaagonist preparations, compared to placebo, in the treatment of adults with chronic obstructive pulmonary disease.
\end{abstract}

Search methods-We searched the Cochrane Airways Group Specialised Register of trials. The date of the most recent search is April 2007.

Selection criteria-Studies were included if they were randomised and double-blind. Studies could compare any combined inhaled corticosteroids and long-acting beta-agonist preparation with placebo.

Data collection and analysis-Two authors independently assessed study risk of bias and extracted data. The primary outcomes were exacerbations, mortality and pneumonia. Healthrelated quality of life (measured by validated scales), lung function and side-effects were

Copyright @ 2010 The Cochrane Collaboration. Published by John Wiley \& Sons, Ltd

Contact address: Luis Javier Nannini, Pulmonary Section, Hospital E Peron, Ruta 11 Y Jm Estrada, G. Baigorria, Santa Fe - Rosario, 2152, Argentina.nanninilj@ cimero.org.ar.

Editorial group: Cochrane Airways Group.

Publication status and date: Edited (no change to conclusions), published in Issue 1, 2010.

Review content assessed as up-to-date: 1 August 2007.

CONTRIBUTIONS OF AUTHORS

LN and PP developed the protocol. Studies were assessed by LN and TJL. TJL and LN checked data and entered into RevMan. TJL and LN conducted the analysis. TJL and LN developed the discussion with input from PP. CJC participated in the 2004 and 2007 updates of the review and offered statistical advice and input with calculating SEMs and SDs for the included studies where appropriate.

\section{DECLARATIONS OF INTEREST}

The authors who have been involved in this review have done so without any known conflicts of interest. None of the authors is considered a paid consultant by any pharmaceutical company which produces agents discussed in this review. 
secondary outcomes. Dichotomous data were analysed as fixed effect odds ratios or rate ratios with $95 \%$ confidence intervals, and continuous data as mean differences and $95 \%$ confidence intervals.

Main results-Eleven studies met the inclusion criteria (6427 participants randomised). Two different combination preparations (fluticasone/salmeterol and budesonide/formoterol) were used. Study quality was good. Fluticasone/salmeterol and budesonide/formoterol both reduced the rate of exacerbations. Pooled analysis of both combination therapies indicated that exacerbations were less frequent when compared with placebo, Rate Ratio: 0.74 (95\% CI 0.7 to 0.8 ). The clinical impact of this effect depends on the frequency of exacerbations experienced by patients. The patients included in these trials had on average 1-2 exacerbations per year which means that treatment with combination therapy would lead to a reduction of one exacerbation every two to four years in these individuals. There is an overall reduction in mortality, but this outcome is dominated by the results of TORCH and further studies on budesonide/formoterol are required. The three year number needed to treat to prevent one extra death is 36 (95\% CI 21 to 258), using a baseline risk of $15.2 \%$ from the placebo arm of TORCH. Both treatments led to statistically significant improvement in health status measurements, although the clinical importance of the differences observed is open to interpretation. Symptoms and lung function assessments favoured combination treatments. There was an increase in the risk of pneumonia with combined inhalers. The three year number needed to treat for one extra case of pneumonia is 13 , using a baseline risk of $12.3 \%$ from the placebo arm of TORCH. Fewer participants withdrew from studies assessing combined inhalers due to adverse events and lack of efficacy.

Authors' conclusions-Compared with placebo, combination therapy led to a significant reduction of a quarter in exacerbation rates. There was a significant reduction in all-cause mortality with the addition of data from the TORCH trial. The increased risk of pneumonia is a concern, and better reporting of this outcome in future studies would be helpful. In order to draw firmer conclusions about the effects of combination therapy in a single inhaler more data are necessary, particularly in relation to the profile of adverse events and benefits in relation to different doses of inhaled corticosteroids.

\section{Medical Subject Headings (MeSH)}

Adrenergic beta-Agonists [*therapeutic use]; Bronchodilator Agents [*therapeutic use]; Drug Combinations; Nebulizers and Vaporizers; Pulmonary Disease, Chronic Obstructive [*drug therapy]; Randomized Controlled Trials as Topic

\section{MeSH check words}

Humans

\section{BACKGROUND}

Both inhaled corticosteroids and long-acting beta-agonists (LABAs) have been shown to be effective in chronic obstructive pulmonary disease (COPD). The evidence available indicates that there is a consistent, statistically significant difference in FEV1 of around 50 $\mathrm{mL}$, and significant differences in health status measurements in favour of salmeterol in patients with a poor response to short-acting bronchodilators (Appleton 2006). Inhaled 
steroids have not been shown to reduce the rate of decline in FEV1 although short-term increases in $\mathrm{FEV}_{1}$ and significant reductions in exacerbations have been reported (Yang 2007). The impact of these effects on morbidity and mortality remain uncertain. LABAs are recommended by NICE 2004 and GOLD (GOLD 2006) when patients continue to experience problems on short-acting bronchodilators.

The evidence base for the addition of long-acting beta-agonists to inhaled steroids in asthma is well established (Ni Chroinin 2004; Ni Chroinin 2005). There are several possible advantages to fixed combination therapy. The most obvious benefit is in terms of patient convenience with the expectation that this may lead to greater treatment adherence. Therefore, it is important to know whether inhaled corticosteroids combined with longacting beta-agonist (LABAs) preparations reduce the number of exacerbations and improve other endpoints such as quality of life, despite the lack of effect on $\mathrm{FEV}_{1}$ decline.

This is an update of a previous review which considered the effect of combination therapy compared with placebo, and both monocomponents in people with chronic obstructive pulmonary disease (COPD) (Nannini 2004). The availability of several new studies has prompted us to split the review between comparisons with placebo and those with monocomponents. This review summarises the evidence from clinical trials comparing combination inhaled steroids and long-acting beta-agonists with placebo. Reviews of combination therapy with inhaled steroids (Nannini 2007a) and long-acting beta-agonists (Nannini 2007b) are published separately. Recent concerns have been raised regarding the safety of long-acting beta-agonists in asthma (Walters 2007) and also the validity of summary estimates from clinical trials which assess exacerbation rates without taking account of follow-up time or adjustment for between-patient variability (Suissa 2006).

\section{OBJECTIVES}

To determine the efficacy of combined inhaled corticosteroid and long-acting beta-agonists for stable COPD in comparison with placebo.

\section{METHODS}

\section{Criteria for considering studies for this review}

Types of studies-Randomised, double-blind clinical trials.

Types of participants-Adult patients (age > 45 years) with known, stable COPD fulfilling American Thoracic Society (ATS), European Respiratory Society (ERS) or Global Initiative for Chronic Obstructive Lung Disease (GOLD) diagnostic criteria. Patients were to be clinically stable, without evidence of an exacerbation for one month prior to study entry. Patients with significant diseases other than COPD, (for example with a diagnosis of asthma, cystic fibrosis, bronchiectasis, or other lung diseases) were excluded. However patients with partial reversibility on pulmonary function testing were included.

\section{Types of interventions}

1. Fluticasone/salmeterol versus placebo 
2. Budesonide/formoterol versus placebo Concomitant therapy was permitted, as long as there was no systematic difference between treatment groups.

\section{Types of outcome measures \\ Primary outcomes}

1. Exacerbations \& hospitalisations

2. Mortality

3. Pneumonia has been added as a primary outcome for this update.

\section{Secondary outcomes}

1. Change in forced expiratory volume in 1 second $\left(\mathrm{FEV}_{1}\right)$ and change in forced ventilatory capacity (FVC): trough, peak and average; and other measures of pulmonary function

2. Exercise performance - six minute walk and other measures

3. Quality of life scales - St George's Respiratory Questionnaire (SGRQ), Chronic Respiratory Disease Questionnaire (CRDQ)

4. Symptoms

5. Inhaled rescue medication used during the treatment period and other concomitant medication usage including antibiotics and steroids

6. Adverse events - palpitations, tremor, hoarseness/dysphonia, oral candidiasis, cataracts, skin bruising, bone fracture, bone density, plasma cortisol level

\section{Search methods for identification of studies}

Electronic searches-Trials were identified using the Cochrane Airways Group Specialised Register of trials, which is derived from systematic searches of bibliographic databases including the Cochrane Central Register of Controlled Trials (CENTRAL), MEDLINE, EMBASE, CINAHL, AMED and PsycINFO, and handsearching of respiratory journals and meeting abstracts. All records in the Specialised Register coded as 'COPD' were searched using the following terms:

(((beta* and agonist*) and long*) or ((beta* and adrenergic*) and long*) and (*steroid or steroid* or corticosteroid*)) or (fluticasone and salmeterol) or Seretide or Advair or (formoterol and budesonide) or Symbicort

The most recent search on the Register was run in April 2007. In addition, we performed a search of LILACS (all years to March 2005) and CENTRAL (The Cochrane Library Issue 1, 2006).

Searching other resources-We reviewed reference lists of all primary studies and review articles for additional references. We also contacted authors of identified randomised trials about other published and unpublished studies. In addition, we consulted the online 
trial registries of GSK and AstraZeneca, manufacturers of FPS and BUDF respectively (www.ctr.gsk.co.uk; www.astrazenecaclinicaltrials.com).

\section{Data collection and analysis}

Selection of studies-Two reviewers independently identified abstracts of trials which appeared potentially relevant. Using the full text of each study, two reviewers independently selected trials for inclusion in the review. Agreement was by simple agreement; third party adjudication was used to resolve differences.

Data extraction and management-Two reviewers independently extracted data from included trials and entered results into the Cochrane Collaboration software program (Review Manager). In some cases, we estimated information regarding outcomes from graphs. This was performed independently by the two reviewers. Data extraction included the following items:

Population: Age, gender, smoking status, study setting (country, practice setting), inclusion and exclusion criteria.

Intervention: Dose, delivery device, duration

Control: Concurrent treatments (ipratropium, beta-2 agonist, inhaled and systemic corticosteroids)

Outcomes: Pulmonary function measures (baseline and follow-up FEV 1 and FVC), timing of pulmonary function measures, 6-minute walk, urgent visits, admissions, self-rated symptom score/symptoms, quality-of-life instruments, adverse events (palpitations, dry mouth, blurred vision, urinary obstruction and constipation), assessors, adjudicator of clinical endpoints. Mortality outcome data were collected from studies of greater than one year's duration where these were available.

Design: method of randomisation, presence and type of run-in period, study design (parallel, cross-over)

Assessment of risk of bias in included studies-We assessed the risk of bias for each study according to the extent to which the design of the trial had protected the findings from bias. Our assessments we made on the basis of allocation, its concealment and blinding.

Dealing with missing data-The reported confidence interval or $\mathrm{P}$ value were used to calculate standard deviations, or standard errors, for results when these were not reported and could not be obtained from the authors of the papers.

Assessment of heterogeneity-For pooled effects, heterogeneity was to be tested using $\mathrm{I}^{2}$ measurement of the degree of variation between the studies, not attributable by the play of chance. 
Data synthesis-For continuous variables, a fixed effects weighted mean difference (MD) was used for outcomes measured on the same metric. Standardised mean difference (SMD) and 95\% confidence interval (CI) was calculated for outcomes where data were combined from studies using different metrics. All similar studies were to be pooled using fixed effect MD/SMD and 95\% CIs.

For dichotomous variables, a fixed effect odds ratio (OR) with 95\% confidence intervals $(95 \% \mathrm{CI})$ were calculated for individual studies. All similar studies were pooled using fixed effect OR and 95\% CIs. Where mean treatment differences were reported, data were entered as generic inverse variance (GIV), provided a standard error for the difference could be extracted or imputed. Where this method was used the effect size was reported from the original papers, for example as Rate Ratio. This method (GIV) was not available when the protocol was written for the review so was not pre-specified.

Subgroup analysis and investigation of heterogeneity-Whilst we separated the type of steroid and long-acting beta-agonist, we pooled studies with differing dosages of the same drug. We planned a priori subgroups as:

1. Disease severity (related to baseline $\mathrm{FEV}_{1}$ and placebo group exacerbation rate) according to the GOLD staging = IIA, IIB (moderate COPD, characterised by deteriorating lung function $(\mathrm{A}=\mathrm{FEV} 1</=80 \%$ predicted; $\mathrm{B}=</=50 \%$ predicted) and progression of symptoms) and III (severe COPD, characterised by severe airflow limitation (FEV1<30\% predicted) and presence of respiratory failure or clinical signs of right heart failure (GOLD 2006).

2. Prior inhaled corticosteroid plus long-acting beta agonists use (dichotomised as yes/no).

3. Concurrent therapy with routine beta-agonist use (short or long-acting), corticosteroid (systemic or inhaled) or theophylline use (dichotomised as yes/no).

4. Reversibility of airflow obstruction with beta-2-agonist therapy (dichotomised as partial/none). Definition: $>12 \%$ and $>200 \mathrm{ml}$ from baseline $\mathrm{FEV}_{1}$ or $>12 \%$ as a per cent of the predicted normal value following MDI salbutamol 200 to 400

5. Dose, duration and delivery method of therapy.

Sensitivity analysis-For pooled effects, heterogeneity was to be tested using $\mathrm{I}^{2}$ measurement of the degree of variation between the studies, not attributable by the play of chance. If heterogeneity was found, ( $\mathrm{I}^{2}$ statistic more than $20 \%$ ), a random effects model was also used to determine the impact of heterogeneity on the overall pooled effect. In addition, the robustness of the results was tested using a sensitivity analysis based on the quality of the trials where possible. 


\section{RESULTS \\ Description of studies}

See: Characteristics of included studies; Characteristics of excluded studies; Characteristics of ongoing studies.

Results of the search-For details of search history, see Table 1. An overview of the division of our original review into three and description of the search results is given in Figure 1.

Included studies-Eleven studies are included in this review. One study is ongoing (Morgan 2004). For a full description of baseline characteristics, methods used and inclusion and exclusion entry criteria. individual studies, see Characteristics of included studies.

Design: All trials had a randomised, double-blind parallel group design. Methods of randomisation were described in one study (Mahler 2002). Method of blinding was not fully described in all studies. Following correspondence from GSK, trial methodology was confirmed for TRISTAN, and AstraZeneca confirmed methodology for Szafranski 2003. Study characteristics were sufficiently described in three data sets without journal publication to justify their inclusion in the review (O'Donnell 2006; SFCT01; SCO100540).

Participants: A total of 6427 participants were randomised to studies in the review. Participants suffered from COPD, with variable definition of COPD and reversibility. COPD was defined by national or international criteria: ATS (Hanania 2003; Mahler 2002) ERS (TORCH; TRISTAN) and GOLD (Barnes 2006; Calverley 2003; Dal Negro 2003; SCO100540; Szafranski 2003). In two studies, definitions were based on lung function tests and smoking history (O'Donnell 2006; SFCT01). Patient populations in the studies suffered from moderate and severe COPD. Hanania 2003 and Mahler 2002 enrolled participants with reversible and non-reversible COPD. In TORCH the participants were not required to have had previous exacerbations requiring oral steroids or antibiotics to be included in the study.

Interventions: All eleven studies compared combination therapy with placebo.

In one study all participants had a two-week run-in treatment with oral corticosteroids, inhaled formoterol and prn SABA (Calverley 2003).

Concomitant therapy was as-needed short-acting beta agonist, or oral steroids and/or antibiotics in the case of exacerbations. However in three studies, theophylline was also used. Eleven percent of participants in Hanania 2003, and all 18 participants in Dal Negro 2003 received theophylline, in addition to the study drugs. The exact proportion of patients in TRISTAN who were taking theophylline was not reported.

In two studies the combination of inhaled corticosteroid/long-acting beta-agonist was fluticasone/salmeterol (FPS) was $250 \mathrm{mcg} / 50 \mathrm{mcg}$ twice daily (Dal Negro 2003; Hanania 2003). In the remainder of the FPS studies the dose was $500 \mathrm{mcg} / 50 \mathrm{mcg}$ twice daily. In 
Calverley 2003 and Szafranski 2003 the combination inhaled corticosteroid/long-acting beta agonist was budesonide/formoterol (BDF) $(320 \mathrm{mcg} / 9 \mathrm{mcg}$ twice daily).

Duration: 13 weeks or less: Barnes 2006; O’Donnell 2006.

24 weeks: Hanania 2003; Mahler 2002; SCO100540.

52 weeks: Calverley 2003; Dal Negro 2003; Szafranski 2003; SFCT01; TRISTAN.

156 weeks: TORCH.

Outcomes: Exacerbations were stratified by medication given (oral steroid and/or antibiotic treatment in Barnes 2006; Calverley 2003; Dal Negro 2003; SCO100540; SFCT01; Szafranski 2003; TORCH; TRISTAN) or hospitalisation (Barnes 2006; Dal Negro 2003; SCO100540; TORCH; TRISTAN). O'Donnell 2006 did not record/report exacerbation data. Hanania 2003 and Mahler 2002 withdrew participants who exacerbated. Lung function was measured as FEV1 or PEF in all the studies. Quality of life assessment by the SGRQ or CRDQ were available for Calverley 2003; Hanania 2003; Kardos 2007; Mahler 2002; SCO100540; SFCT01; Szafranski 2003; TORCH; TRISTAN. All cause mortality was reported by TORCH.

Excluded studies-Studies that did not meet the entry criteria of this review are listed in Characteristics of excluded studies.

\section{Risk of bias in included studies}

Our assessments of the risk of bias for allocation and blinding are provided in Figure 2. The majority of our judgements on allocation procedures were unclear due to paucity of this information in the trial reports. We were able to ascertain that these were at a low risk of bias in three large studies (Szafranski 2003; TORCH; TRISTAN). All studies used identical inhaler devices to deliver therapy in the treatment groups.

\section{Effects of interventions}

See Summary of Findings table (See Figure 3).

\section{Primary outcomes: Exacerbations, Mortality and Pneumonia Rate of exacerbations}

FPS versus placebo: There was a significant reduction in the rate of exacerbations with combination therapy when compared with placebo $(0.74 ; 95 \%$ CI 0.69 to 0.8 , three trials, $\mathrm{N}$ $=4222$ ). This result was not altered by removing TRISTAN whose summary estimate may have been biased by inadequate adjustment for between patient variability (Suissa 2006), see Figure 4. Additional analysis was performed on exacerbations by specific definitions. Compared with placebo, FPS led to fewer exacerbations requiring oral steroids (rate ratio $0.57 ; 95 \%$ CI 0.52 to 0.63 , three studies), less requirement for antibiotics (rate ratio $0.695 \%$ CI 0.43 to 0.84 , one study) and fewer hospitalisations (rate ratio 0.83 ; $95 \%$ CI 0.7 to 0.97 , two studies). 
BDF versus placebo: There was a significant effect on pooled exacerbation rates in favour of $\mathrm{BDF}$ when expressed as a rate ratio compared with placebo $(0.74 ; 95 \%$ CI 0.62 to 0.88$)$ (see Figure 4). These results are based on 913 participants from two trials (Calverley 2003; Szafranski 2003).

When combined together the overall reduction in rate ratio of exacerbations using either FPS or $\mathrm{BDF}$ is $0.74 ; 95 \%$ CI 0.69 to 0.79 , five trials, $\mathrm{N}=5135$.

\section{Dichotomous data (number of people experiencing an exacerbation)}

FPS versus placebo: No significant difference: OR 0.91; 95\% CI 0.69 to 1.19, four studies, $\mathrm{N}=1109$.

\section{Mortality}

FPS versus placebo: The adjusted hazard ratio from TORCH did not identify a significant effect of FPS over placebo $(0.825,0.681$ to $1.002, \mathrm{P}=0.052)$. Analysed as number of deaths in each treatment group by odds ratio and combined with data from other four other studies, there was a significant reduction in the odds of death in favour of FPS versus placebo (Odds Ratio $0.79,95 \%$ CI 0.65 to $0.98, \mathrm{~N}=4829$ ). Data were separated according to the time point and subgrouped for data at three years, data reported at 1-3 years, data at 1 year and data at 6 months.

BDF versus placebo: The two studies of one year duration did not detect a significant difference between BDF and placebo. The point estimate (Odds Ratio $0.78,95 \% \mathrm{CI} 0.35$ to 1.73) was similar to that for FPS and the was no significant difference in the odds ratio for mortality between FPS and BDF.

When combined together the overall reduction in the odds ratio for mortality with either FPS or BDF was $0.79,95 \% \mathrm{CI} 0.65$ to $0.96, \mathrm{~N}=5752$, see Figure 5. Since differing lengths of follow-up across the studies hinders the calculation of a pooled NNT, we have tabulated this for each study individually (see Table 2). The three year NNT (using the baseline risk of $15.2 \%$ in the placebo arm of TORCH) to prevent one extra death is 36 (95\% CI 21 to 258).

\section{Pneumonia}

FPS versus placebo: Addition of data from TORCH shows a significant increase in pneumonia with FPS in comparison with placebo. The pooled OR is 1.80 (95\% CI 1.48 to 2.18).

BDF versus placebo: Calverley 2003 reported data on pneumonia and although the increase was not statistically significant (OR 4.13, 95\% CI 0.87 to 19.64) the direction of effect was similar to FPS and there is no significant difference between the increase in pneumonia when BDF is compared to FPS.

For combined inhalers, the pooled OR for pneumonia is 1.83 (95\% CI 1.51 to 2.21). Table 3 gives the range of $\mathrm{NNT}(\mathrm{H})$ across the studies for pneumonia. A pooled NNT(H) was not calculated due to the wide differences in duration and the likely impact this would have on the calculation of a pooled event rate. The three year NNT(H) (using the baseline risk of 
$12.3 \%$ in the combination therapy arm of $\mathrm{TORCH}$ ) for one extra patient to suffer pneumonia was 13.

\section{Secondary outcomes}

Quality of life

\section{St George Respiratory Questionnaire (results collected from end of study)}

FPS versus placebo: Treatment with FPS improved SGRQ scores by an average of -2.9 units versus placebo (95\% CI: -3.61 to -2.18 , four studies, $\mathrm{N}=3346$ ).

BDF versus placebo: There was a significant effect in favour of BDF compared with placebo of -6.06 units on the SGRQ (95\%CI -7.90 to -4.22$)$. There was a high level of heterogeneity when these data were pooled (I square 71.8\%). Random effects modelling also generated a significant effect (MD -5.8 units [95\%CI -9.32 to -2.28$]$ ). The magnitude of improvement in the Szafranski 2003 BDF group was 3.9 units from baseline, and was not dissimilar to the change scores from post run-in treatment in the Calverley 2003 (see graphical presentation of data in the published article, P 916). However, the placebo group deteriorated more in Calverley 2003, which possibly reflects the withdrawal of active treatment, with the subsequent loss of the pre-dosing effects achieved with high dose OCS and LABAs. In comparison, BDF may have maintained the pre-dosing treatment effects of QoL more successfully.

\section{Chronic Respiratory Disease Questionnaire (results collected from end of study)}

FPS versus placebo: Pooled data from Mahler 2002 and Hanania 2003 indicated a statistically significant improvement in CRDQ for those treated with FPS compared with placebo (5 units (95\% CI 2.48 to 7.52 ).

\section{Symptom score}

FPS versus placebo: FPS led to improved symptom scores (transitional dysponea index) when compared with placebo (MD 1.04 (95\% CI 0.56 to 1.53) Mahler 2002 and Hanania 2003 gave a significant difference in change scores in favour FPS .

TRISTAN reported improvements in symptoms after treatment in favour of FPS versus placebo on breathlessness scores $(\mathrm{P}<0.001)$, and night time awakenings (FPS mean number of nights per week: 2.31; placebo mean: $3.01(\mathrm{P}=0.006)$. Cough scores also favoured FPS $(\mathrm{P}=0.018)$.

BDF versus placebo: Data were pooled for Calverley 2003 and Szafranski 2003. There was a significant effect in favour of BDF when compared with placebo, MD -0.63 (95\% CI -0.90 to -0.37$)$. 


\section{Lung function}

\section{Predose \& post-dose $\mathrm{FEV}_{\mathbf{1}}$ - Change from baseline}

FP versus placebo: Pooled analysis of data were conducted without findings from the Dal Negro 2003 study. Owing to the small size of this study, we were concerned that the SDs represented an inaccurate estimate for the SDs of the population, and the small variance increased the weight of the study, out of all proportion to its size. Data pooled from seven studies gave a MD in predose $\mathrm{FEV}_{1}$ of 0.16 Litres; $95 \%$ CI 0.14 to $0.19, \mathrm{~N}=1408$. Analysis of post-dose $\mathrm{FEV}_{1}$ from TORCH indicated a significant improvement in favour of FPS over placebo of $0.09 \mathrm{~L}$.

BDF versus placebo: $\mathrm{FEV}_{1}$ data were reported as $\%$ change. There was a significant increase in $\mathrm{FEV}_{1}$ in favour of BDF versus placebo of $14.40 \%$ (95\% CI 11.91 to 16.90 , two studies).

\section{Predose $\mathrm{FEV}_{\mathbf{1}}$-Absolute values}

FPS versus placebo: TRISTAN and SFCT01 reported data as mL and Dal Negro 2003 reported $\%$ predicted.

The results of this analysis have not been pooled due to concern over the small number of similar patients in Dal Negro 2003, and the very low variance. It is therefore not appropriate to combine the results on an SMD scale.

Pooled data from TRISTAN and SFCT01 led to a significant difference in favour of FPS of 0.13 Litres; $95 \%$ CI 0.10 to 0.16 .

\section{Rescue medication}

FPS versus placebo: Pooled data from Mahler 2002 and Hanania 2003 indicated a significant reduction in mean puffs per day of short-acting beta-agonist usage for FPS versus placebo (MD -1.19 puffs/day; 95\% CI: -1.83 to -0.55 ).

Mahler 2002 reported significant increases in the percentage of nights with no awakenings requiring short-acting beta-agonist in favour of FPS versus placebo (5.7\% versus $-4.3 \%$ respectively, $\mathrm{P}<0.031$ ).

TRISTAN reported a significant difference in median $\%$ of days without use of relief medication: FPS: $14 \%$ versus placebo: $0 \%(\mathrm{P}<0.001)$.

BDF versus placebo: $\mathrm{BDF}$ treatment reduced the requirement for reliever medication when compared with placebo. The combined results of Calverley 2003; Szafranski 2003, MD -0.87 puffs per day $(95 \% \mathrm{CI}-1.15$ to -0.58$), \mathrm{I}^{2} 29.1 \%$; Random effects modelling: MD -0.92 puffs per day $(95 \% \mathrm{CI}-1.34$ to -0.50$)$.

\section{Safety \& tolerability}

FPS versus placebo: There was no significant difference in the occurrence of overall reported adverse events between FPS and placebo: OR 1.10; 95\% CI 0.96 to 1.27; eight studies, $\mathrm{N}=5493$. 
Pneumonia, candidiasis, nasopharyngitis, hoarseness and upper respiratory tract infections (URTI) occurred more frequently in FPS treated participants:

Pneumonia: OR 1.80; $95 \%$ CI 1.48 to 2.18; seven studies, $\mathrm{N}=5229$.

Candidiasis: OR 5.73; 95\%CI 3.07 to 10.67; six studies, $\mathrm{N}=1958$.

Hoarseness: OR 8.79; 95\% CI 1.11 to 69.62 ; two studies, $\mathrm{N}=585$.

Nasopharyngitis: OR 1.28 ; $95 \%$ CI 1.05 to 1.56 ; two studies, $\mathrm{N}=3535$.

URTI: OR $1.23 ; 95 \%$ CI 1.04 to 1.47 ; five studies, $\mathrm{N}=4963$.

\section{Withdrawals}

FPS versus placebo: There were significantly fewer withdrawals from treatment with FPS than placebo (OR $0.69 ; 95 \%$ confidence interval 0.62 to 0.78 ). Withdrawals due to adverse events and lack of efficacy also occurred less frequently on treatment with FPS than placebo (withdrawal due to adverse event: OR 0.76 ; $95 \%$ confidence interval 0.65 to 0.88 ; eight studies, 5110 participants; withdrawal due to lack of efficacy: OR 0.30; $95 \%$ confidence interval 0.21 to 0.42 ; five studies, 4620 participants).

BDF versus placebo: Data were pooled from Calverley 2003 and Szafranski 2003 for withdrawals due to worsening COPD symptoms and adverse events.

There was a significant difference in withdrawals due to worsening of COPD symptoms when BDF was compared with placebo, OR 0.40 [95\%CI 0.28 to 0.58].

There was no significant difference between BDF and placebo on the likelihood withdrawal due to adverse events other than COPD deterioration, OR 1.30 [95\% CI 0.78 to 2.18].

\section{DISCUSSION}

We have reviewed data from 11 randomised controlled trials (6427 participants) assessing the effectiveness of combined inhaled corticosteroid and long-acting beta-agonist in the treatment of chronic obstructive pulmonary disease (COPD). Whilst the consensus regarding the definitions of COPD and COPD exacerbations evolve, the trial evidence to date indicates that in severe COPD where this is defined by low FEV1, significant smoking history, and a recent history of reduced disease control there is a role for combination therapy in reducing exacerbations which lead to unscheduled additional treatment, including courses of oral steroid therapy and hospitalisation.

\section{Patient populations}

There is much debate as whether 'chronic obstructive pulmonary disease' includes patients who have a significant bronchodilator response to short-acting beta-agonists, although recent definitions permit some reversibility. In this review, we have attempted to stratify data according to baseline reversibility. There is debate as to whether such a distinction is a clinically valid dichotomy (Burge 2003; Calverley 2003a). We have opted to pool data from 
both these populations as there remains little consensus on this issue (ATS 1995), but for some outcomes (such as $\mathrm{FEV}_{1}$ ) a sub-group analysis has been carried out on the basis of reversibility. It is also of interest that Calverley 2003 and Szafranski 2003 were classified as low reversibility on the basis of change in FEV1 following short acting beta-agonists, but nevertheless achieved a mean change in FEV1 of around 15\% following pre-treatment with oral steroids and long-acting beta-agonist treatment in the studies.

Baseline FEV1 data from the studies indicate that the effects observed in the analyses were measured in populations who were over-whelmingly severe. Intention-to-treat analyses were conducted in all the studies, but on outcomes such as mean exacerbation rates, the withdrawal of severe frequent exacerbators in the studies may have distorted study findings due to the lower exacerbation rates on active treatment. The loss of these patients from the studies may thus limit the accuracy of mean event rates.

There was also a high proportion of current smokers in Mahler 2002 and TRISTAN. A recent study has demonstrated a diminished response to dexamethasone in vitro following the simulation of smoking effects. Smoke effects induced the inhibition of the histone deacetylases (HDAC) enzyme which is necessary in the process of gene repression (Ito 2001). Whilst one in vivo study has demonstrated the resistance to steroid action in asthmatic smokers (Pedersen 1996), there may nevertheless be a similar inhibition of the expression of corticosteroids when inhaled or systemic steroids are used in current smokers with COPD, resulting in compromised corticosteroid effectiveness.

\section{Clinical endpoints}

The outcomes identified a priori to be of primary significance related to exacerbations and hospitalisations. Limited data were presented in the trials on hospitalisations as a separate outcome although the evidence available did indicate that combination treatment reduced the rate of this event. Previous studies have indicated that ICS reduces the mean rate of exacerbations requiring oral steroid or antibiotics in patients with moderate and severe COPD (Yang 2007). However, the dose at which ICS is considered to efficacious, and the patients most likely to respond to this therapy remain unclear. The magnitude of these differences and their clinical impact depends upon the frequency of patients exacerbations. In severe patients with a history of one or more exacerbations in the previous year, the difference of $24 \%$ may translate to one exacerbation less every 4 years. Further assessment of the impact of the combination therapy upon hospitalisations and exacerbations is required.

There is growing interest in the definition and analysis of exacerbations which occur in clinical trials of therapies in COPD (Roisin 2000; Suissa 2006; Calverley 2005). The role of any maintenance therapy in this population should be assessed in terms of the burden of deteriorating disease control to both the individual patient and the healthcare system which rationalises resources in treating the debilitating effects of chronic disease progression. The relationship between event defined exacerbations (such as short courses of oral steroids or antibiotics, unscheduled presentation) and other markers of health such as symptoms and quality of life continue to present trialists and guideline developers with the dilemma of when to recommend initiating additional therapy based on the trial evidence available, and 
the extent to which varying definitions of endpoint alter external validity in applying evidence to the needs of an individual patient. The question of exacerbations, and the appropriate statistical analysis of rate ratios, casts some doubt over the validity of some of the findings in this review. In particular, the large long-term studies (i.e. those in excess of six months) which are adequately powered to detect statistically significant findings may overestimate the treatment effects of this therapy (Suissa 2006). The method of weighting counts of exacerbations described by Suissa 2006 (using duration of person follow-up time as a denominator in calculating the mean group rate of exacerbations rather than an unweighted approach) was undertaken in Calverley 2003; Szafranski 2003 and TORCH. In these studies the effects were consistent and significantly favoured combination therapy over placebo.

Mortality was recorded in many of the studies, but was collected and analysed as a primary outcome in TORCH. Cause-specific mortality was reported, but the definition of a primary cause of death continues to pose challenges in a population of patients who suffer from comorbidities such as lung cancer (McGarvey 2007). The findings in the original trial publication as a hazard ratio (HR). The HR reported in TORCH was adjusted for repeated measurements, and was not statistically significant (ratio: $0.825, \mathrm{P}=0.052$ ). The significant finding in our review of a ratio of odds of death overall of 0.79 in favour of treatment probably reflects the fact that our analyses were not subject to adjustment for repeated measurement. Furthermore, this estimate encompasses data from studies with considerably shorter lengths of follow-up which provide only limited long-term prognostic value. This explains why TORCH provides 53\% participants, and $89 \%$ of the weight. The three year NNT (from the baseline risk in TORCH) to prevent one extra death is 36 (95\% CI 21 to 258).

Both the CRDQ and the SGRQ were used to measure the effect of combination therapy on health status. There was a high level of statistical variation in the FPS studies for total SGRQ scores, although the reasons for this are not clear. Differing treatment protocols in the two budesonide/formoterol combination studies may explain the differences between health status scores. Although differences between combination therapy and placebo in Calverley 2003 and Szafranski 2003 were statistically significant, the difference from Calverley 2003 suggested that combination treatment led to a clinically meaningful advantage compared with placebo (i.e. greater than 4 units, Jones 2002). This difference should be interpreted with some caution due to the effects of pre-treatment with high dose prednisolone and formoterol, which led to a clinically meaningful increase of 4.5 units on the SGRQ. These effects were sustained with combination treatment when compared with placebo, but statistical tests on the comparisons with component therapy were not available.

Side-effects were more common on treatment than placebo. The increased risk of pneumonia with combination therapy is of particular concern since this frequently leads to hospitalisation in elderly patients. The association between prescription of inhaled fluticasone and pneumonia in people with COPD has been identified from a case-control study (Ernst 2007). The rate ratio from that study indicated an increase in risk of pneumonia leading to hospitalisation of 70\% (RR 1.7). From RCT data in this review the combination of salmeterol and fluticasone also led to an increase in the odds of pneumonia of 1.8 
(representing an increase in the risk of pneumonia of 65\%). The NNT(h) for this outcome varies considerably, with three long-term studies (i.e. one year or greater) showing the lowest NNT(H) (Calverley 2003: 36; TORCH: 13; TRISTAN: 153, see Table 3).

The advantages associated with combination therapy extend beyond potentially greater patient compliance with a convenient delivery system of two therapies. The component treatments have complementary mechanisms of action, whereby the smooth muscle relaxation of the beta-agonist combined with the anti-inflammatory action of the steroid offer greater scope of clinical benefits than they would do alone. Cazzola 2000 reported a single-blind trial assessing the additive benefit of long-acting beta-agonist and inhaled steroids. When administered in separate inhalers there was no significant improvement in lung function until three months of treatment. The results from the trials in this review have not addressed the question of synergistic effects, and further research comparing combination therapy in a single versus separate inhaler devices could elucidate this issue more clearly.

Fixed dose therapy may be an effective pharmacological strategy in clinically stable moderately severe COPD, but if patients become unstable other approaches would be needed (GOLD 2006). Consensus guidelines recommend oral corticosteroids and/or antibiotics depending on the cardinal symptoms of the exacerbation of COPD (GOLD 2006). At this time it would be inappropriate to manage a clinically unstable situation with combined fixed therapy on its own.

\section{AUTHORS' CONCLUSIONS}

\section{Implications for practice}

In participants with moderate and severe COPD, there is clinical benefit when long acting beta-agonist and inhaled corticosteroid are co-administered compared to treatment with placebo, with fewer exacerbations and better quality of life. The effect on all cause mortality with active treatment is statistically significant. Fluticasone/salmeterol also led to increased harm with an increased risk of pneumonia with active treatment. In order to draw firmer conclusions about the effects of combination therapy in a single inhaler, assessment of the comparative effects of different doses of inhaled corticosteroids is necessary. Ongoing and future trials should allow better clarification of any additional benefits of combined therapy.

\section{Implications for research}

Assessment of budesonide/formoterol in large trials is required to assess whether this preparation confers similar benefits when compared with placebo on mortality. Combined therapy should be compared with separate administration of long acting beta-agonist and inhaled corticosteroid at different doses in a double-dummy design study in large scale multi-centre studies, in order to assess whether combined therapy confers benefits over the simple addition of beta-agonist to different doses of inhaled steroid treatment in separate inhalers. This should include an evaluation of patient compliance. A pharmacoeconomic analysis would be very helpful to assist purchasers of healthcare. Documentation of serious adverse events such as hospitalisation, intensive care support and also standardised 
collection and reporting of deaths and pneumonia would offer very valuable insights in to the long-term effects of this form of therapy.

\section{Acknowledgments}

The authors are indebted to the Hamamellis Trust who very generously funded the return travel for Dr Nannini to London in order to spend a week working on the development of the review. Thanks to Liz Arnold, Susan Hansen and Veronica Stewart for advice and support in developing our literature searching strategy and for retrieval of full text papers. We would also like to acknowledge the efforts of Inge Vestbo, Diane Grimley and Karen Richardson of GSK who helped us in our attempts to obtain unpublished information on the TRISTAN and Wouters 2005 studies, and those of Goran Tornling, Moira Coughlan and Roger Metcalf of AstraZeneca in helping us obtain data for Szafranski 2003. We thank Dr Nick Hanania and Prof Donald Mahler for corresponding with us in our attempts to obtain unpublished data from their studies, and Dr Dal Negro (Dal Negro 2003) for confirming the SDs in his study. We are grateful to Nancy Santesso for preparing the summary of findings table for our review.

\section{SOURCES OF SUPPORT}

Internal sources

- Hamamellis Trust, UK.

- $\quad$ NHS Cochrane Grant scheme, UK.

External sources

- $\quad$ No sources of support supplied

\section{CHARACTERISTICS OF STUDIES}

\section{Characteristics of included studies [ordered by study ID]}

Barnes 2006

\begin{tabular}{|c|c|}
\hline Methods & $\begin{array}{l}\text { Parallel group design } \\
\text { Randomisation: not clear } \\
\text { Blinding: double blind, identical inhaler devices used } \\
\text { Allocation concealment: unclear } \\
\text { Excluded: not described } \\
\text { Withdrawals: described } \\
\text { Trial duration: } 13 \text { weeks } \\
\text { Baseline characteristics: comparable } \\
\text { Intention to treat analysis stated } \\
\text { Jadad Score: } 4\end{array}$ \\
\hline \multirow[t]{5}{*}{ Participants } & Setting: 18 centres in Western and Eastern Europe. \\
\hline & $\begin{array}{l}2 \text { Participants randomised: } 141 \text { (two groups: FP/SAL combination: 74; } \\
\text { placebo: 67). }\end{array}$ \\
\hline & $\begin{array}{l}3 \text { Baseline characteristics: } 64 \text { years. mean FEV1 L: } 1.68 \text {; mean FEV1 \% } \\
\text { predicted: 59; mean FEV1 reversibility: } 3.9 \text { (of predicted) }\end{array}$ \\
\hline & $\begin{array}{l}4 \text { Inclusion criteria: M/F } 40-80 \text { years of age; diagnosis of COPD } \\
\text { (according to GOLD criteria); }>/=2 \text { on MRC dyspnea scale; poor } \\
\text { reversibility of }<10 \% \text { predicted normal. }\end{array}$ \\
\hline & $\begin{array}{l}5 \text { Exclusion criteria: Current diagnosis of asthma; recent exacerbation } \\
\text { (within } 4 \text { weeks); LTOT; Pulmonary rehabilitation; ICS, anti-leukotriene } \\
\text { or tiotropium within } 14 \text { days of visit } 1\end{array}$ \\
\hline \multirow[t]{3}{*}{ Interventions } & Run-in phase: 4 weeks. Treatment during this phase of the study not described \\
\hline & $1 \quad$ FPS 500/50mcg bid. \\
\hline & $2 \quad$ Placebo \\
\hline
\end{tabular}


Inhaler device: DPI

\begin{tabular}{|c|c|c|}
\hline Outcomes & \multicolumn{2}{|c|}{ Exacerbations; withdrawals; adverse events } \\
\hline \multicolumn{3}{|l|}{ Notes } \\
\hline \multicolumn{3}{|l|}{ Risk of bias } \\
\hline Item & Authors' judgement & Description \\
\hline Adequate sequence generation? & Unclear & $\begin{array}{l}\text { Described as randomised; no other } \\
\text { information available }\end{array}$ \\
\hline Allocation concealment? & Unclear & Information not available \\
\hline $\begin{array}{l}\text { Blinding? } \\
\text { All outcomes }\end{array}$ & Yes & Identical inhaler devices \\
\hline
\end{tabular}

Calverley 2003

\begin{tabular}{|c|c|}
\hline Methods & $\begin{array}{l}\text { Parallel group study. } \\
\text { Randomisation: unclear } \\
\text { Blinding: double-blind (identical inhaler devices) } \\
\text { Trial duration: } 52 \text { weeks with two week run-in of treatment optimisation. } \\
\text { Allocation concealment: unclear } \\
\text { Withdrawals: stated } \\
\text { Intention to treat analysis: stated } \\
\text { Jadad score: } 4\end{array}$ \\
\hline \multirow[t]{5}{*}{ Participants } & Setting: 109 centres in 15 countries \\
\hline & $\begin{array}{l}2 \text { Participants randomised: } 510 \text { (BDF: 254; PLA: 256) Additional } \\
\text { treatment groups not covered in this review: BUD: 257; F: } 255\end{array}$ \\
\hline & $\begin{array}{l}3 \text { Baseline characteristics: mean age: 64; mean FEV1 L: 1; mean FEV1 \% } \\
\text { predicted: 36; mean SGRQ: } 48\end{array}$ \\
\hline & $\begin{array}{l}\text { Inclusion criteria: GOLD defined COPD (stages III and IV); }>/=40 \\
\text { years; COPD symptoms }>2 \text { years; smoking history }>/=10 \text { pack years; } \\
\text { FEV1/VC }</=70 \% \text { pre-BD; FEV } 1</=50 \% \text { predicted; use of SABAs as } \\
\text { reliever medication; }>/=1 \text { COPD exacerbation requiring OCS/antibx } \\
2-12 \text { months before } 1 \text { st clinic visit. }\end{array}$ \\
\hline & $\begin{array}{l}\text { Exclusion criteria: History of asthma/rhinitis before } 40 \text { years of age; any } \\
\text { relevant cardiovascular disorders; exacerbation of COPD requiring } \\
\text { medical intervention within } 4 \text { weeks of run-in/during run-in phase; non- } \\
\text { allowed medications: O2 therapy; ICS - (aside from study medication), } \\
\text { disodium cromoglycate, leukotriene-antagonists, } 5 \text {-LO inhibitors, BD } \\
\text { (other than study medication and prn terbutaline } 0.5 \mathrm{mg} \text { ), antihistamines, } \\
\text { medication containing ephedrine, B-blocking agents }\end{array}$ \\
\hline Interventions & $\begin{array}{l}\text { Run-in phase: All participants received } 30 \mathrm{mg} \text { oral prednisolone BiD and } 2 \times 4.5 \mathrm{mg} \\
\text { formoterol BiD } \\
\text { (2 weeks) } \\
\text { 1) BDF: } 320 / 9 \mathrm{mcg} \text { bid. } \\
\text { 2)Placebo (lactose monohydrate). } \\
\text { Additional treatment groups not covered in this review: } \\
\text { 3) BUD: } 400 \mathrm{mcg} \text { bid. } \\
\text { 3) F: } 9 \text { mcg bid. } \\
\text { Inhaler device: Turbuhaler. }\end{array}$ \\
\hline Outcomes & $\begin{array}{l}\text { Time to first exacerbation; change in post-medication FEV1; number of } \\
\text { exacerbations; time to and number of OCS-treated episodes; am and pm PEF, slow } \\
\text { VC, HRQL, symptoms, use of reliever medication, AEs }\end{array}$ \\
\hline Notes & $\begin{array}{l}\text { Classified as 'poorly reversible population'. } \\
\text { P values used to calculate pooled SEMs for the following outcomes: Health related } \\
\text { quality of life; } \\
\text { FEV1; rescue medication }\end{array}$ \\
\hline Kisk oJ & \\
\hline
\end{tabular}

Cochrane Database Syst Rev. Author manuscript; available in PMC 2014 September 15. 


\begin{tabular}{lll} 
Item & Authors' judgement & Description \\
\hline Adequate sequence generation? & Unclear & $\begin{array}{l}\text { Described as randomised; no other } \\
\text { information available }\end{array}$ \\
\hline Allocation concealment? & Unclear & Information not available \\
\hline $\begin{array}{l}\text { Blinding? } \\
\text { All outcomes }\end{array}$ & Yes & Identical inhaler devices \\
\hline
\end{tabular}

Dal Negro 2003

\begin{tabular}{|c|c|c|}
\hline Methods & \multicolumn{2}{|c|}{$\begin{array}{l}\text { Parallel group study. } \\
\text { Randomisation: unclear } \\
\text { Blinding: double-blind } \\
\text { Method of randomisation: Not reported. } \\
\text { Allocation concealment: unclear } \\
\text { Trial duration: } 52 \text { weeks. } \\
\text { Withdrawals: Stated. } \\
\text { Baseline characteristics: Comparable Intention to treat analysis: Yes } \\
\text { Jadad score: } 3\end{array}$} \\
\hline \multirow[t]{5}{*}{ Participants } & 1 & Setting: Single centre in Italy \\
\hline & 2 & $\begin{array}{l}\text { Participants randomised: } 12 \text { (FPS: 6; PLA: 6) Additional treatment } \\
\text { groups not covered in this review: SAL: } 6\end{array}$ \\
\hline & 3 & $\begin{array}{l}\text { Baseline characteristics: Age range: } 53-78 \text {; moderate COPD; mean FEV1 } \\
\text { (L): 1.46; mean FEV1 (\% predicted): 48; mean PEF (L/min): 180; mean } \\
\text { reversibility ( } \% \text { baseline): } 3.2\end{array}$ \\
\hline & 4 & $\begin{array}{l}\text { Inclusion criteria: baseline FEV1 } \% \text { predicted: }</=80 \% \text {; FEV1 }>800 \mathrm{~mL} \text {; } \\
\text { FEV1/FVC ratio: }</=70 \% \text { predicted; FEV1 change of }</=12 \% \\
\text { predicted post } 400 \mathrm{mg} \text { SAL; regular treatment with oral theophylline } \\
\text { 20mg BiD; SABA prn (for at least } 6 \text { months); current/ex-smokers with } \\
\text { smoking history of at least } 10 \text { pack years. }\end{array}$ \\
\hline & 5 & $\begin{array}{l}\text { Exclusion criteria: Current evidence of asthma or other pulmonary } \\
\text { diseases; regular treatment with ICS; unstable respiratory disease } \\
\text { requiring oral/parenteral CS within } 4 \text { weeks prior to the beginning of the } \\
\text { study; changes in COPD medication in last } 4 \text { weeks before entering run- } \\
\text { in; upper/lower respiratory tract infection within } 4 \text { weeks before last } \\
\text { screening visit; unstable angina/unstable arrhythmias; recent MI/heart } \\
\text { failure; insulin-dependent diabetes mellitus; neuropsychiatric disorders; } \\
\text { concurrent use of medications that affect COPD (e.g. B-blockers), or } \\
\text { interact with methylxanthine products, e.g. macrolides or } \\
\text { fluoroquinolones; known/suspected hypersensitivity to ICS, B-agonist or } \\
\text { lactose; evidence of alcohol abuse }\end{array}$ \\
\hline \multirow[t]{6}{*}{ Interventions } & \multicolumn{2}{|c|}{ Run-in: 2 weeks treatment with theophylline and prn SABA. } \\
\hline & 1 & FPS 50/250 mcg bid. \\
\hline & 2 & Placebo. \\
\hline & & Additional treatment groups not covered in this review \\
\hline & 3 & SAL 50mcg bid. \\
\hline & \multicolumn{2}{|c|}{$\begin{array}{l}\text { Participants were on concomitant therapy: SABA prn and theophylline } 400 \mathrm{ug} / \text { day, } \\
\text { for } 12 \text { months } \\
\text { Inhaler device: Diskus. }\end{array}$} \\
\hline Outcomes & \multicolumn{2}{|c|}{$\begin{array}{l}\text { FEV1, Delta FEV1, PEF am, symptom scores, rescue medication use, exacerbations } \\
\text { (event rate and mean number per year) }\end{array}$} \\
\hline Notes & \multicolumn{2}{|c|}{$\begin{array}{l}\text { Classified as 'poorly reversible population'. Jadad score: } 3 \\
\text { Mild exacerbation: requirement for increase in SABA prn by }>2 \text { occasions } / 24 \mathrm{hrs} \text { on } \\
\text { two or more consecutive days compared with baseline mean of last seven days of } \\
\text { run-in } \\
\text { Moderate exacerbation: condition requiring treatment with antibiotics and/or oral } \\
\text { corticosteroids }\end{array}$} \\
\hline
\end{tabular}

Cochrane Database Syst Rev. Author manuscript; available in PMC 2014 September 15. 
Severe exacerbation: Condition requiring emergency hospital treatment and/or hospitalisation

\begin{tabular}{lll}
\hline Risk of bias & & \\
\hline Item & Authors' judgement & Description \\
\hline Adequate sequence generation? & Unclear & $\begin{array}{l}\text { Described as randomised; no other } \\
\text { information available }\end{array}$ \\
\hline Allocation concealment? & Unclear & Information not available \\
\hline $\begin{array}{l}\text { Blinding? } \\
\text { All outcomes }\end{array}$ & Yes & Identical inhaler devices \\
\hline
\end{tabular}

Hanania 2003

\begin{tabular}{|c|c|c|c|}
\hline Methods & \multicolumn{3}{|c|}{$\begin{array}{l}\text { Parallel group study. } \\
\text { Randomisation: method unclear. } \\
\text { Blinding: double blind. } \\
\text { Allocation concealment: unclear } \\
\text { Excluded: described. } \\
\text { Withdrawals: described. } \\
\text { Trial duration: } 24 \text { weeks with 2-week run-in period. } \\
\text { Baseline characteristics: comparable. } \\
\text { Intention to treat analysis: not stated. } \\
\text { Jadad score: } 4\end{array}$} \\
\hline \multirow[t]{5}{*}{ Participants } & 1 & \multicolumn{2}{|c|}{ Setting: USA, Multi-centre (76 hospitals) } \\
\hline & 2 & \multicolumn{2}{|c|}{$\begin{array}{l}\text { Patients randomised: } 368 \text { (FPS: 183; PLA: 185). Additional treatment } \\
\text { groups not covered in this review; SAL: } 177 ; \text { FP: } 183\end{array}$} \\
\hline & 3 & \multicolumn{2}{|c|}{$\begin{array}{l}\text { Baseline characteristics: Mean age: } 64 \text {; mean FEV1: 1.27L ( } 42 \% \\
\text { predicted) }\end{array}$} \\
\hline & 4 & \multicolumn{2}{|c|}{$\begin{array}{l}\text { Inclusion criteria: stable COPD, FEV } 140-65 \% \text { predicted, FEV1/FVC }< \\
70 \% \text { predicted, symptoms of chronic bronchitis and moderate dyspnoea }\end{array}$} \\
\hline & 5 & \multicolumn{2}{|c|}{$\begin{array}{l}\text { Exclusion criteria: current diagnosis of asthma, use of oral steroids in } \\
\text { past } 6 \text { weeks, abnormal ECG, LTOT, moderate - severe exacerbation in } \\
\text { run in. Other significant medical disorder }\end{array}$} \\
\hline \multirow[t]{7}{*}{ Interventions } & \multicolumn{3}{|c|}{ Run-in: 2 weeks treatment with placebo inhaler and prn SABA. } \\
\hline & 1 & \multicolumn{2}{|c|}{ FPS 50/250 mcg bid. } \\
\hline & 2 & \multicolumn{2}{|l|}{ Placebo } \\
\hline & & \multicolumn{2}{|c|}{ Additional treatment groups not covered in this review } \\
\hline & 3 & \multicolumn{2}{|c|}{ SAL 50 mcg bid. } \\
\hline & 4 & \multicolumn{2}{|c|}{ FP 250 mcg bid. } \\
\hline & \multicolumn{3}{|c|}{ Inhaler device: Diskus. } \\
\hline Outcomes & \multicolumn{3}{|c|}{$\begin{array}{l}\text { Lung function: Change in FEV1 from baseline to end of study (M). PEF data not } \\
\text { stratified by reversibility. Quality of life: CRDQ, CBSQ not stratified by } \\
\text { reversibility. Dyspnoea and symptoms: } \\
\text { Transitional dyspnoea index, Baseline dyspnoea index not stratified by reversibility. } \\
\text { Exacerbations. } \\
\text { Rescue salbutamol use }\end{array}$} \\
\hline Notes & \multicolumn{3}{|c|}{$\begin{array}{l}\text { FEV } 1 \text { reversibility }<12 \% \text { or } 200 \mathrm{ml} \text { (of baseline FEV1) Reversibility stratified data. } \\
\text { Mean } \% \text { increase non-reversible patients }=8.8\end{array}$} \\
\hline \multicolumn{4}{|l|}{ Risk of bias } \\
\hline Item & \multicolumn{3}{|c|}{ Authors' judgement $\quad$ Description } \\
\hline Adequate sequence generation? & Unclear & & $\begin{array}{l}\text { Described as randomised; no other } \\
\text { information available }\end{array}$ \\
\hline
\end{tabular}




\begin{tabular}{lll} 
Allocation concealment? & Unclear & Information not available \\
\hline $\begin{array}{l}\text { Blinding? } \\
\text { All outcomes }\end{array}$ & Yes & Identical inhaler devices \\
\hline
\end{tabular}

\section{Mahler 2002}

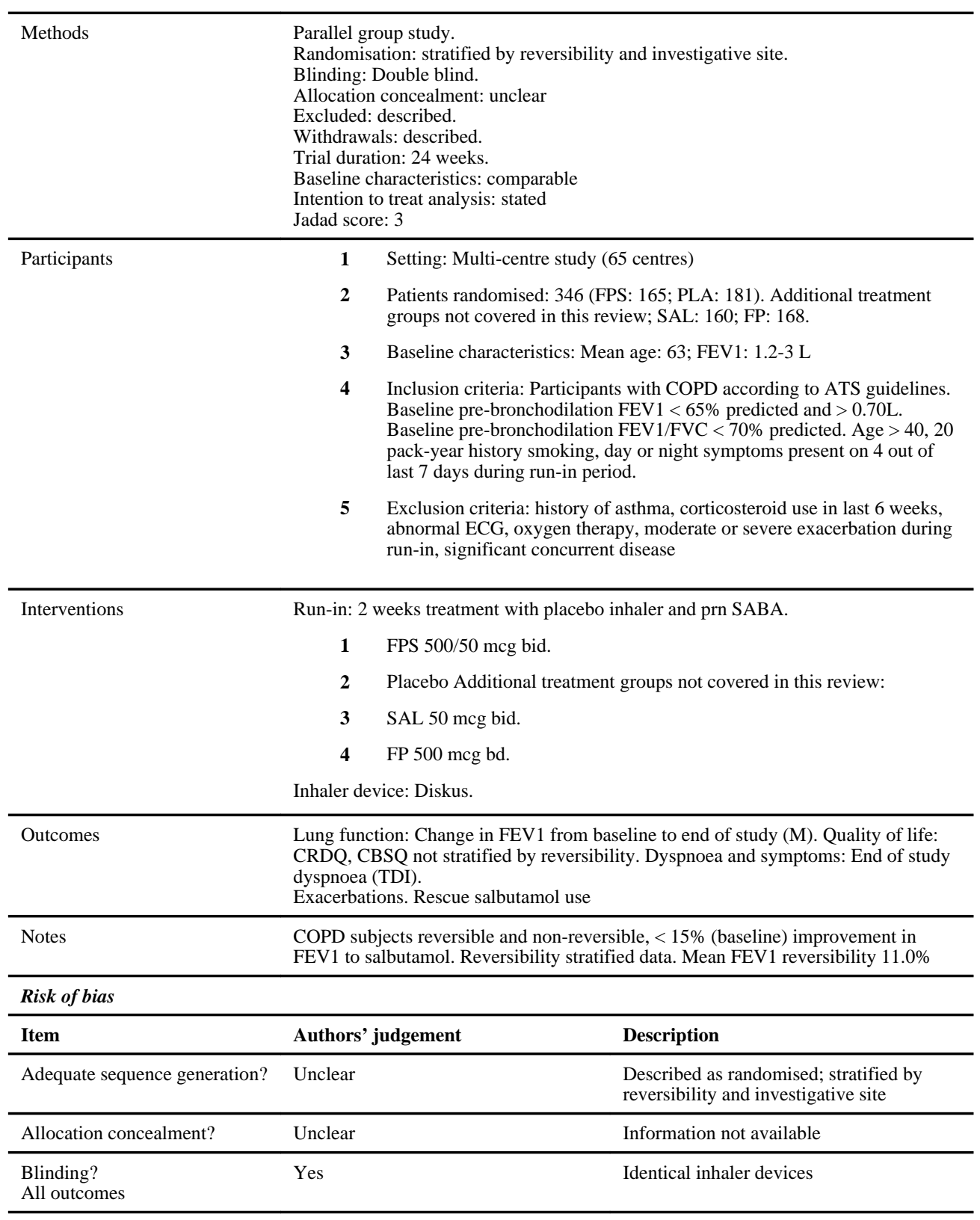

O’Donnell 2006

Cochrane Database Syst Rev. Author manuscript; available in PMC 2014 September 15. 


\begin{tabular}{|c|c|c|c|}
\hline Methods & \multicolumn{3}{|c|}{$\begin{array}{l}\text { Parallel group design } \\
\text { Randomisation: not clear } \\
\text { Blinding: double blind } \\
\text { Allocation concealment: unclear } \\
\text { Excluded: not described } \\
\text { Withdrawals: described } \\
\text { Trial duration: } 8 \text { weeks } \\
\text { Baseline characteristics: comparable } \\
\text { Intention to treat analysis stated } \\
\text { Jadad Score: } 3\end{array}$} \\
\hline \multirow[t]{5}{*}{ Participants } & 1 & \multicolumn{2}{|c|}{ Setting: 22 centres in North America } \\
\hline & 2 & \multicolumn{2}{|c|}{$\begin{array}{l}\text { Participants randomised: } 126 \text { (FP/SAL: } 62 \text {; placebo: } 64 \text { ) Additional } \\
\text { treatment groups not covered in this review: salmeterol: } 59\end{array}$} \\
\hline & 3 & \multicolumn{2}{|c|}{ Baseline characteristics: 65 years; FEV1: 1.12L } \\
\hline & 4 & \multicolumn{2}{|c|}{$\begin{array}{l}\text { Inclusion criteria: } \mathrm{M} / \mathrm{F}>/=40 \text { years of age; diagnosis of } \mathrm{COPD} ;>/=10 \\
\text { pack year; baseline Borg dyspnoea index }<7 \text {; FEV1 }<70 \% \text { predicted; } \\
\text { FRC }>/=120 \% \text { predicted }\end{array}$} \\
\hline & 5 & \multicolumn{2}{|c|}{$\begin{array}{l}\text { Exclusion criteria: Current diagnosis of asthma; use of xanthines/ } \\
\text { LABAs/OCS/ICS }\end{array}$} \\
\hline \multirow[t]{5}{*}{ Interventions } & \multicolumn{3}{|c|}{ Run-in: 2 weeks. Single-blind placebo. } \\
\hline & 1 & \multicolumn{2}{|c|}{ FPS 500/50mcg bid. } \\
\hline & 2 & \multicolumn{2}{|c|}{ Placebo Additional treatment groups not covered in this review: } \\
\hline & 3 & \multicolumn{2}{|c|}{ SAL 50mcg bid. } \\
\hline & \multicolumn{3}{|c|}{ Inhaler device: DPI. } \\
\hline Outcomes & \multicolumn{3}{|c|}{ Withdrawals; exercise time; FEV1; adverse events } \\
\hline Notes & \multicolumn{3}{|c|}{ Study downloaded from ctr.gsk.co.uk } \\
\hline \multicolumn{4}{|l|}{ Risk of bias } \\
\hline Item & \multicolumn{2}{|c|}{ Authors' judgement } & Description \\
\hline Adequate sequence generation? & Unclear & & $\begin{array}{l}\text { Described as randomised; no other } \\
\text { information available }\end{array}$ \\
\hline Allocation concealment? & Unclear & & Information not available \\
\hline $\begin{array}{l}\text { Blinding? } \\
\text { All outcomes }\end{array}$ & Yes & & Identical inhaler devices \\
\hline
\end{tabular}

SCO100540

\begin{tabular}{|c|c|c|}
\hline Methods & \multicolumn{2}{|c|}{$\begin{array}{l}\text { Parallel group design } \\
\text { Randomisation: not clear } \\
\text { Blinding: double blind (identical inhaler devices) } \\
\text { Allocation concealment: unclear } \\
\text { Excluded: not described } \\
\text { Withdrawals: described } \\
\text { Trial duration: } 24 \text { weeks } \\
\text { Baseline characteristics: comparable } \\
\text { Intention to treat analysis: stated } \\
\text { Jadad Score: } 4\end{array}$} \\
\hline \multirow[t]{3}{*}{ Participants } & 1 & Setting: 12 centres in China \\
\hline & 2 & $\begin{array}{l}\text { Subjects randomised: } 445 \text { (two groups: FP/SAL combination: 297; PLA: } \\
\text { 148) }\end{array}$ \\
\hline & 3 & Baseline characteristics: 66 years; Male: $89 \%$ \\
\hline
\end{tabular}

Cochrane Database Syst Rev. Author manuscript; available in PMC 2014 September 15. 
4 Inclusion criteria: M/F 40-80 years of age; diagnosis of COPD according to GOLD criteria; $<10 \%$ reversibility of predicted FEV1; FEV1/FVC ratio $<70 \%$; FEV $1<60 \%$ predicted

5 Exclusion criteria: Respiratory disease other than COPD; requirement for $>12 \mathrm{hrs} /$ day LTOT;

Received $>1000 \mathrm{mcg} / \mathrm{d}$ BDP; $500 \mathrm{mcg} / \mathrm{d}$ FP in 4 weeks prior to run-in; hospitalisation with exacerbation of COPD

Interventions

Run-in phase: 2 weeks. Treatment during this phase of the study not described

1 FP/SAL combination 500/50mcg BID

2 Placebo

Inhaler device: DPI

\begin{tabular}{lll}
\hline Outcomes & $\begin{array}{l}\text { FEV1; SGRQ; Symptoms; Bronchodilator usage; Exacerbations (requirement for } \\
\text { antibiotics; OCS and hospitalisations); withdrawals; adverse events }\end{array}$ \\
\hline Notes & Study downloaded from ctr.gsk.co.uk & \\
\hline Risk of bias & & Description \\
\hline Item & Authors' judgement & $\begin{array}{l}\text { Described as randomised; no other } \\
\text { information available }\end{array}$ \\
\hline Adequate sequence generation? & Unclear & Information not available \\
\hline Allocation concealment? & Unclear & Identical inhaler devices \\
\hline $\begin{array}{l}\text { Blinding? } \\
\text { All outcomes }\end{array}$ & Yes & \\
\hline
\end{tabular}

\section{SFCT01}

\begin{tabular}{|c|c|c|}
\hline Methods & \multicolumn{2}{|c|}{$\begin{array}{l}\text { Parallel group design. } \\
\text { Randomisation: not clear. } \\
\text { Blinding: double blind. } \\
\text { Allocation concealment: unclear. } \\
\text { Excluded: not described. } \\
\text { Withdrawals: described. } \\
\text { Trial duration: } 52 \text { weeks. } \\
\text { Baseline characteristics: comparable. Intention to treat analysis stated. Jadad Score: } 3\end{array}$} \\
\hline \multirow[t]{5}{*}{ Participants } & 1 & Setting: 49 centres in Italy, 7 in Poland. \\
\hline & 2 & $\begin{array}{l}\text { Participants randomised: } 256 \text { (FP/SAL: } 131 \text {; placebo: } 125) \text { Additional } \\
\text { treatment groups not covered in this review: fluticasone: } 131 \text {. }\end{array}$ \\
\hline & 3 & Baseline characteristics: 65 years; FEV1: not reported. \\
\hline & 4 & $\begin{array}{l}\text { Inclusion criteria: M/F }>/=40 \text { years of age; diagnosis of COPD; }>/=10 \\
\text { pack year; FEV1 }<70 \% \text { predicted and }>800 \mathrm{ml} \text {; Reversibility }<10 \% \\
\text { predicted normal (and }<200 \mathrm{ml} \text { ). }\end{array}$ \\
\hline & 5 & Exclusion criteria: Not described. \\
\hline \multirow[t]{5}{*}{ Interventions } & \multicolumn{2}{|c|}{ Run-in: 2 weeks. All maintenance LABA and ICS treatment ceased } \\
\hline & 1 & FPS 500/50mcg bid. \\
\hline & 2 & Placebo Additional treatment groups not covered in this review: \\
\hline & 3 & FP 500mcg bid. \\
\hline & \multicolumn{2}{|c|}{ Inhaler device: MDI } \\
\hline Outcomes & \multicolumn{2}{|c|}{ Withdrawals; exacerbations; FEV1; adverse events } \\
\hline Notes & \multicolumn{2}{|c|}{ Unpublished study downloaded from ctr.gsk.co.uk } \\
\hline
\end{tabular}




\begin{tabular}{lll} 
Risk of bias & Authors' judgement & Description \\
\hline Item & Unclear & $\begin{array}{l}\text { Described as randomised; no other } \\
\text { information available }\end{array}$ \\
\hline Adequate sequence generation? & Information not available \\
\hline Allocation concealment? & Unclear & Identical inhaler devices \\
\hline $\begin{array}{l}\text { Blinding? } \\
\text { All outcomes }\end{array}$ & Yes & \\
\hline
\end{tabular}

\section{Szafranski 2003}

Methods
Parallel group study.
Randomisation: Randomised, double-blind, placebo-controlled parallel group trial.
Duration: 52 weeks.
Methods of randomisation: Computer-generated scheme at AstraZeneca, Lund,
Sweden. At each centre, eligible patients received an enrolment code and then after
run-in, participants were allocated the next consecutive patient number.
Allocation concealment: adequate.
Blinding: All the Turbuhaler inhalers were identical to ensure that the patient,
pharmacist and the investigator were blinded to the allocated treatment
Excluded: Not stated
Withdrawals: Stated
Intention to treat analysis: Stated

Participants 1 Setting: 89 centres in Central \& South America, Europe and South Africa.

2 Participants: 413 (BDF: 208; PLA: 205). Additional treatment groups not covered in this review: F: 201; BUD: 198.

3 Baseline characteristics: Mean age: 64 years; mean FEV1 \% predicted: $36 \%$, mean reversibility $6 \%$ predicted normal.

4 Inclusion criteria: Age $>/=40$ years; $\operatorname{COPD}$ for $>/=2$ years; smoking history $>/=10$ pack years; FEV $1</=50 \%$ predicted; FEV1/FVC $<1$ $=70 \%$; Symptom score $>/=2$ during at least 7 days of run-in; use of bronchodilators for reliever medication; $>/=1$ severe COPD exacerbation within 212 months before study entry.

5 Exclusion criteria: history of asthma/rhinitis before age of 40; using betablockers; current respiratory tract disease other than COPD

\begin{tabular}{|c|c|c|}
\hline \multirow[t]{5}{*}{ Interventions } & Run-in: & weeks. Treatment with prn SABA only. \\
\hline & 1 & BDF $320 / 9$ mcg bid. \\
\hline & 2 & Placebo Additional treatment groups not covered in this review: \\
\hline & 3 & BUD 400ug bid. \\
\hline & 4 & F 9ug bid. \\
\hline
\end{tabular}

Inhaler device: Turbuhaler

\begin{tabular}{lll}
\hline Outcomes & Symptoms, adverse events, exacerbations, lung function. \\
\hline Notes & $\begin{array}{l}\text { Classified as 'poorly reversible' subgroup. Jadad score: 5. Exacerbation defined as } \\
\text { requirement of oral steroids and/or antibiotics and/or hospitalisation for respiratory } \\
\text { symptoms. Mild exacerbation defined as requirement of }>/=4 \text { inhalations per day } \\
\text { P values used to calculate pooled SEMs for following outcomes: Symptoms; rescue } \\
\text { medication usage }\end{array}$ \\
\hline Risk of bias & Authors' judgement & Description \\
\hline Item & Yes & Computer-generated scheme \\
\hline Adequate sequence generation? & Yes & $\begin{array}{l}\text { At each centre, eligible patients received an } \\
\text { enrolment code and then after run-in, }\end{array}$
\end{tabular}

Cochrane Database Syst Rev. Author manuscript; available in PMC 2014 September 15. 
participants were allocated the next consecutive patient number

\begin{tabular}{lll} 
& & consecutive patient number \\
\hline $\begin{array}{l}\text { Blinding? } \\
\text { All outcomes }\end{array}$ & Yes & Identical inhaler devices \\
\hline
\end{tabular}

TORCH

\begin{tabular}{|c|c|c|c|}
\hline Methods & \multicolumn{3}{|c|}{$\begin{array}{l}\text { Parallel group design } \\
\text { Randomisation: Permuted block randomisation with stratification for smoking status } \\
\text { and country } \\
\text { Blinding: double blind (identical inhaler devices) } \\
\text { Allocation concealment: Adequate } \\
\text { Excluded: described } \\
\text { Withdrawals: described } \\
\text { Trial duration: } 156 \text { weeks } \\
\text { Baseline characteristics: comparable } \\
\text { Intention to treat analysis: stated } \\
\text { Jadad Score: } 5\end{array}$} \\
\hline \multirow[t]{5}{*}{ Participants } & 1 & \multicolumn{2}{|c|}{ Setting: 444 centres in North America, Central America and Asia Pacific } \\
\hline & 2 & \multicolumn{2}{|c|}{$\begin{array}{l}\text { Subjects randomised: } 3091 \text { (FP/SAL: } 1546 \text {; PLA: } 1545) \text { Additional } \\
\text { treatment groups not covered in this review: SAL: } 1542 \text {; FP: } 1551 .\end{array}$} \\
\hline & 3 & \multicolumn{2}{|c|}{ Baseline characteristics: 65 years; Male: $76 \%$} \\
\hline & 4 & \multicolumn{2}{|c|}{$\begin{array}{l}\text { Inclusion criteria: M/F } 40-80 \text { years of age; diagnosis of COPD (ERS); } \\
<10 \% \text { reversibility of predicted FEV1; FEV1/FVC ratio }<70 \% \text {; FEV1< } \\
60 \% \text { predicted; }>/=10 \text { pack year smoking history }\end{array}$} \\
\hline & 5 & \multicolumn{2}{|c|}{$\begin{array}{l}\text { Exclusion criteria: Asthma or respiratory diseases other than COPD; } \\
\text { LVRS/lung transplant; requirement for > } 12 \mathrm{hrs} / \text { day LTOT; long term } \\
\text { OCS therapy; serious uncontrolled disease likely to interfere with } \\
\text { medication/cause death in next three years" }\end{array}$} \\
\hline \multirow[t]{6}{*}{ Interventions } & \multicolumn{3}{|c|}{ Run-in: 2 weeks. All maintenance treatment with ICS and LABA ceased } \\
\hline & 1 & \multicolumn{2}{|c|}{ FP/SAL combination 500/50mcg BID } \\
\hline & 2 & \multicolumn{2}{|c|}{ Placebo Additional treatment groups not covered in this review: } \\
\hline & 3 & \multicolumn{2}{|c|}{ FP 500mcg BID } \\
\hline & 4 & \multicolumn{2}{|c|}{ SAL 50mcg BID } \\
\hline & \multicolumn{3}{|c|}{ Inhaler device: DPI } \\
\hline Outcomes & \multicolumn{3}{|c|}{$\begin{array}{l}\text { All cause mortality; change in SGRQ; exacerbations (requiring antibiotics, steroids, } \\
\text { hospitalisation or combination of these); lung function; withdrawals; adverse events }\end{array}$} \\
\hline \multicolumn{4}{|l|}{ Notes } \\
\hline \multicolumn{4}{|l|}{ Risk of bias } \\
\hline Item & \multicolumn{3}{|c|}{ Authors' judgement $\quad$ Description } \\
\hline Adequate sequence generation? & \multicolumn{2}{|l|}{ Yes } & $\begin{array}{l}\text { Computer generated scheme. Permuted block } \\
\text { randomisation with stratification for smoking } \\
\text { status and country }\end{array}$ \\
\hline Allocation concealment? & \multicolumn{3}{|r|}{ Centralised randomisation schedule } \\
\hline $\begin{array}{l}\text { Blinding? } \\
\text { All outcomes }\end{array}$ & \multicolumn{3}{|r|}{ Identical inhaler devices } \\
\hline
\end{tabular}

TRISTAN

Cochrane Database Syst Rev. Author manuscript; available in PMC 2014 September 15. 


\begin{tabular}{|c|c|c|c|}
\hline Methods & \multicolumn{3}{|c|}{$\begin{array}{l}\text { Parallel group design. } \\
\text { Randomisation: computer generated. Numbers were generated off-site. Once a } \\
\text { treatment number had been assigned to a participant it could not be assigned to any } \\
\text { other participant. } \\
\text { Blinding: Double blind. Participants received identically packaged and presented } \\
\text { placebos. } \\
\text { Excluded: Described. } \\
\text { Withdrawals: Described. } \\
\text { Trial duration: } 2 \text { week run-in period, } 52 \text { weeks treatment, } 2 \text {-week follow-up } \\
\text { Baseline characteristics: Comparable Intention to treat analysis: stated } \\
\text { Jadad Score: } 5\end{array}$} \\
\hline \multirow[t]{5}{*}{ Participants } & 1 & \multicolumn{2}{|c|}{ Setting: 196 centres in Europe, South Africa and Australia. } \\
\hline & 2 & \multicolumn{2}{|c|}{$\begin{array}{l}\text { Participants randomised: } 719 \text { (FPS: 358; PLA: 361) Additional treatment } \\
\text { groups not covered in this review: SAL: 372; FP: } 375 \text {. }\end{array}$} \\
\hline & 3 & \multicolumn{2}{|c|}{$\begin{array}{l}\text { Baseline characteristics: Mean age } 63 \text { years, mean FEV1 }=1.26 \mathrm{~L}(44 \% \\
\text { predicted). }\end{array}$} \\
\hline & 4 & \multicolumn{2}{|c|}{$\begin{array}{l}\text { Inclusion criteria: Baseline FEV1 } 25-75 \% \text { predicted; FEV1/FVC ratio } \\
</=70 \% \text {; Poor reversibility: < } 10 \% \text { increase of predicted FEV1 } 30 \\
\text { minutes after inhaling } 400 \text { mcg salbutamol; at least } 10 \text { pack years } \\
\text { smoking history; history of exacerbations (at least } 1 \text { in the last year) } \\
\text { requiring OCS and/or antibiotics. At least one episode of acute COPD } \\
\text { per year in the previous } 3 \text { years. }\end{array}$} \\
\hline & 5 & \multicolumn{2}{|c|}{$\begin{array}{l}\text { Exclusion criteria: respiratory disorders other than COPD. Oxygen } \\
\text { treatment, systemic corti-costeroids, high doses of inhaled corticosteroids } \\
(>1000 \mathrm{mcg} \text { daily beclomethasone dipropionate, budesonide or } \\
\text { flunisolide or }>500 \mathrm{mcg} \text { daily fluticasone) or antibiotics in the four } \\
\text { weeks before the } 2 \text { week run-in period }\end{array}$} \\
\hline \multirow[t]{6}{*}{ Interventions } & \multicolumn{3}{|c|}{ Run-in: 2 weeks. All maintenance treatment with ICS and LABA ceased } \\
\hline & 1 & \multicolumn{2}{|c|}{ FPS $50 \mathrm{mcg} / 500 \mathrm{mcg}$ bid. } \\
\hline & 2 & \multicolumn{2}{|c|}{ Placebo Additional treatment groups not covered in this review: } \\
\hline & 3 & \multicolumn{2}{|c|}{ SAL 50 mcg bid. } \\
\hline & 4 & \multicolumn{2}{|c|}{ FP 500 mcg bid. } \\
\hline & \multicolumn{3}{|c|}{ Inhaler device: DPI } \\
\hline Outcomes & \multicolumn{3}{|c|}{$\begin{array}{l}\text { FEV1; PEF; exercise tolerance; quality of life: SGRQ; dyspnoea and symptoms } \\
\text { (symptom score for shortness of breath, cough and sputum production); } \\
\text { exacerbations (defined as requirement for antibiotics, oral steroids or both); rescue } \\
\text { salbutamol use }\end{array}$} \\
\hline Notes & \multicolumn{3}{|c|}{ FEV1 reversibility (\% predicted normal) Mean Reversibility $(\%$ predicted $)=3.8$} \\
\hline \multicolumn{4}{|l|}{ Risk of bias } \\
\hline Item & \multicolumn{3}{|c|}{ Authors' judgement } \\
\hline Adequate sequence generation? & Yes & & $\begin{array}{l}\text { Computer generated randomisation } \\
\text { schedule. }\end{array}$ \\
\hline Allocation concealment? & Yes & & $\begin{array}{l}\text { Numbers were generated off-site. Once a } \\
\text { treatment number had been assigned to a } \\
\text { participant it could not be assigned to any } \\
\text { other participant }\end{array}$ \\
\hline $\begin{array}{l}\text { Blinding? } \\
\text { All outcomes }\end{array}$ & Yes & & Identical inhaler device \\
\hline
\end{tabular}

BD: bronchodilator; BDF: Budesonide/formoterol combination; bid: twice daily; BUD: Busesonide; CBSQ: Chronic bronchitis symptom questionnaire; CRDQ: Canadian respiratory disease questionnaire; F: Formoterol; FEV1: Forced expiratory volume in one second; FP: Fluticasone; FPS: Fluticasone/salmeterol combination; FVC: Forced vital capacity; ICS: inhaled corticosteroid; LABA: long acting beta agonist; LTOT: Long-term oxygen therapy; MMRC: Modified Medical Research Council; OCS: oral corticosteroids; PLA: Placebo; PRN: as needed; SABA: short acting beta agonist SAL: Salmeterol; SGRQ: St George respiratory questionnaire; 


\section{Characteristics of excluded studies [ordered by study ID]}

\begin{tabular}{ll}
\hline Study & Reason for exclusion \\
\hline Aaron 2004 & Irrelevant comparison \\
\hline Borgstrom 2003 & Healthy volunteers \\
\hline Cazzola 2000 & $\begin{array}{l}\text { Single-blind assessment of additive benefit of inhaled fluticasone to salmeterol. Although dosage } \\
\text { was identical to Seretide/Advair (ie FP 500ug: SAL 50mcg), treatment was administered through } \\
\text { separate inhalers }\end{array}$ \\
\hline Cazzola 2002a & $\begin{array}{l}\text { Single-blind randomised crossover study comparing combination salmeterol and fluticasone with } \\
\text { formoterol and budesonide - excluded as duration of study was too short (12 hours) }\end{array}$ \\
\hline Cazzola 2003 & Acute phase COPD. \\
\hline Cazzola 2004 & $\begin{array}{l}\text { Randomised trial comparing combination salmeterol/fluticasone with separately administered } \\
\text { fluticasone and theophylline for 4 months. Excluded as the comparison was not within the scope of } \\
\text { the review }\end{array}$ \\
\hline Cazzola 2004b & The duration of this study was too short (<1 week). \\
\hline Chapman 2002 & Review article. \\
\hline Donohue 2004 & Irrelevant comparison \\
\hline Kardos 2007 & Comparison of combination therapy long-acting beta-agonist \\
\hline Noschese 2003 & Non-randomised study. \\
\hline SAM40116 & $\begin{array}{l}\text { Within study treatment group imbalances in dosage of steroids/combination therapy based upon } \\
\text { historical steroid dose }\end{array}$ \\
\hline SCO100470 & Comparison of combination therapy long-acting beta-agonist \\
\hline SCO40034 & Comparison of tiotropium and combination therapy \\
\hline Soriano 2002 & Non-randomised retrospective survival analysis. \\
\hline Sun 2004 & Irrelevant comparison \\
\hline Vestbo 2004 & Review article. \\
\hline Study excluded as it assessed the withdrawal of FP from combination therapy \\
\hline
\end{tabular}

\section{Characteristics of ongoing studies [ordered by study ID]}

Morgan 2004

\begin{tabular}{ll}
\hline Trial name or title & None given \\
\hline Methods & \\
\hline Participants & People with COPD \\
\hline Interventions & Combination versus single administration of ICS and LABA \\
\hline Outcomes & \\
\hline Starting date & \\
\hline Contact information & \\
\hline Notes
\end{tabular}


DATA AND ANALYSES

Comparison 1

Combined inhalers versus Placebo (Primary Outcomes)

\begin{tabular}{|c|c|c|c|c|}
\hline Outcome or subgroup title & No. of studies & No. of participants & Statistical method & Effect size \\
\hline $\begin{array}{l}1 \text { Exacerbation Rates with } \\
\text { combined inhalers v. placebo }\end{array}$ & 5 & & $\begin{array}{l}\text { Rate Ratio (Fixed, 95\% } \\
\text { CI) }\end{array}$ & $0.74[0.69,0.79]$ \\
\hline 1.1 Fluticasone/salmeterol & 3 & & $\begin{array}{l}\text { Rate Ratio (Fixed, 95\% } \\
\text { CI) }\end{array}$ & $0.74[0.69,0.80]$ \\
\hline 1.2 Budesonide/formoterol & 2 & & $\begin{array}{l}\text { Rate Ratio (Fixed, 95\% } \\
\text { CI) }\end{array}$ & $0.74[0.62,0.88]$ \\
\hline 2 Mortality & 7 & 5752 & $\begin{array}{l}\text { Odds Ratio (M-H, } \\
\text { Fixed, } 95 \% \mathrm{CI})\end{array}$ & $0.79[0.65,0.96]$ \\
\hline 2.1 Fluticasone/salmeterol & 5 & 4829 & $\begin{array}{l}\text { Odds Ratio (M-H, } \\
\text { Fixed, } 95 \% \mathrm{CI})\end{array}$ & $0.79[0.65,0.97]$ \\
\hline 2.2 Budesonide/formoterol & 2 & 923 & $\begin{array}{l}\text { Odds Ratio (M-H, } \\
\text { Fixed, } 95 \% \mathrm{CI})\end{array}$ & $0.78[0.35,1.73]$ \\
\hline 3 Pneumonia & 8 & 5739 & $\begin{array}{l}\text { Odds Ratio (M-H, } \\
\text { Fixed, } 95 \% \mathrm{CI})\end{array}$ & $1.83[1.51,2.21]$ \\
\hline 3.1 FPS & 7 & 5229 & $\begin{array}{l}\text { Odds Ratio (M-H, } \\
\text { Fixed, } 95 \% \mathrm{CI})\end{array}$ & $1.80[1.48,2.18]$ \\
\hline $3.2 \mathrm{BDF}$ & 1 & 510 & $\begin{array}{l}\text { Odds Ratio (M-H, } \\
\text { Fixed, } 95 \% \text { CI) }\end{array}$ & $4.13[0.87,19.64]$ \\
\hline
\end{tabular}

Comparison 2

Fluticasone/salmeterol (FPS) versus placebo (PLA)

\begin{tabular}{|c|c|c|c|c|}
\hline Outcome or subgroup title & No. of studies & No. of participants & Statistical method & Effect size \\
\hline $\begin{array}{l}1 \text { Number of participants with } \\
\text { one or more exacerbation }\end{array}$ & 5 & 1235 & $\begin{array}{l}\text { Odds Ratio (M-H, } \\
\text { Fixed, } 95 \% \mathrm{CI})\end{array}$ & $0.88[0.67,1.15]$ \\
\hline 1.1 Reversible population & 1 & 126 & $\begin{array}{l}\text { Odds Ratio (M-H, } \\
\text { Fixed, } 95 \% \mathrm{CI})\end{array}$ & $0.32[0.06,1.66]$ \\
\hline $\begin{array}{l}1.2 \text { Partially reversible } \\
\text { population (mixed population) }\end{array}$ & 2 & 713 & $\begin{array}{l}\text { Odds Ratio (M-H, } \\
\text { Fixed, } 95 \% \mathrm{CI})\end{array}$ & $1.00[0.69,1.44]$ \\
\hline $\begin{array}{l}1.3 \text { Poorly reversible } \\
\text { population }\end{array}$ & 2 & 396 & $\begin{array}{l}\text { Odds Ratio (M-H, } \\
\text { Fixed, } 95 \% \mathrm{CI})\end{array}$ & $0.80[0.53,1.21]$ \\
\hline $\begin{array}{l}2 \text { End of treatment mean } \\
\text { number of exacerbations per } \\
\text { participant }\end{array}$ & 1 & & $\begin{array}{l}\text { Mean Difference } \\
(\mathrm{IV}, \text { Fixed, } 95 \% \mathrm{CI})\end{array}$ & Totals not selected \\
\hline $\begin{array}{l}\text { 2.1 Partially reversible } \\
\text { population (mixed population) }\end{array}$ & 0 & & $\begin{array}{l}\text { Mean Difference } \\
(\mathrm{IV}, \text { Fixed, } 95 \% \mathrm{CI})\end{array}$ & Not estimable \\
\hline $\begin{array}{l}2.2 \text { Poorly reversible } \\
\text { population }\end{array}$ & 1 & & $\begin{array}{l}\text { Mean Difference } \\
\text { (IV, Fixed, 95\% CI) }\end{array}$ & Not estimable \\
\hline 3 Exacerbations & 3 & & $\begin{array}{l}\text { Rate ratio (Fixed, } \\
95 \% \mathrm{CI} \text { ) }\end{array}$ & $0.74[0.69,0.80]$ \\
\hline $\begin{array}{l}\text { 3.1 Partially reversible } \\
\text { population (mixed population) }\end{array}$ & 0 & & $\begin{array}{l}\text { Rate ratio (Fixed, } \\
95 \% \mathrm{CI} \text { ) }\end{array}$ & Not estimable \\
\hline $\begin{array}{l}3.2 \text { Poorly reversible } \\
\text { population }\end{array}$ & 3 & & $\begin{array}{l}\text { Rate ratio (Fixed, } \\
95 \% \mathrm{CI} \text { ) }\end{array}$ & $0.74[0.69,0.80]$ \\
\hline $\begin{array}{l}4 \text { Number of exacerbations by } \\
\text { type }\end{array}$ & 2 & & $\begin{array}{l}\text { Odds Ratio (M-H, } \\
\text { Fixed, } 95 \% \mathrm{CI})\end{array}$ & Subtotals only \\
\hline $\begin{array}{l}\text { 4.1 Requirement for oral } \\
\text { steroids }\end{array}$ & 2 & 417 & $\begin{array}{l}\text { Odds Ratio (M-H, } \\
\text { Fixed, } 95 \% \mathrm{CI})\end{array}$ & $1.01[0.61,1.68]$ \\
\hline
\end{tabular}




\begin{tabular}{|c|c|c|c|c|}
\hline Outcome or subgroup title & No. of studies & No. of participants & Statistical method & Effect size \\
\hline $\begin{array}{l}4.2 \text { Requirement for } \\
\text { antibiotic treatment }\end{array}$ & 1 & 140 & $\begin{array}{l}\text { Odds Ratio (M-H, } \\
\text { Fixed, } 95 \% \text { CI) }\end{array}$ & $0.80[0.26,2.44]$ \\
\hline $\begin{array}{l}4.3 \text { Requirement for oral } \\
\text { steroid or antibiotic treatment }\end{array}$ & 1 & 140 & $\begin{array}{l}\text { Odds Ratio (M-H, } \\
\text { Fixed, } 95 \% \text { CI) }\end{array}$ & $3.32[0.13,82.80]$ \\
\hline 4.4 Hospitalisation & 1 & 140 & $\begin{array}{l}\text { Odds Ratio (M-H, } \\
\text { Fixed, } 95 \% \mathrm{CI})\end{array}$ & $3.32[0.13,82.80]$ \\
\hline 5 Exacerbations by type & 3 & & $\begin{array}{l}\text { Rate ratio (Fixed, } \\
95 \% \mathrm{CI} \text { ) }\end{array}$ & Subtotals only \\
\hline $\begin{array}{l}5.1 \text { Requirement for oral } \\
\text { steroids }\end{array}$ & 3 & & $\begin{array}{l}\text { Rate ratio (Fixed, } \\
95 \% \mathrm{CI} \text { ) }\end{array}$ & $0.57[0.52,0.63]$ \\
\hline $\begin{array}{l}5.2 \text { Requirement for } \\
\text { antibiotic treatment }\end{array}$ & 1 & & $\begin{array}{l}\text { Rate ratio (Fixed, } \\
95 \% \mathrm{CI} \text { ) }\end{array}$ & $0.60[0.43,0.84]$ \\
\hline $\begin{array}{l}5.3 \text { Requirement for oral } \\
\text { steroid or antibiotic treatment }\end{array}$ & 0 & & $\begin{array}{l}\text { Rate ratio (Fixed, } \\
95 \% \mathrm{CI} \text { ) }\end{array}$ & Not estimable \\
\hline 5.4 Hospitalisation & 2 & & $\begin{array}{l}\text { Rate ratio (Fixed, } \\
95 \% \mathrm{CI} \text { ) }\end{array}$ & $0.83[0.70,0.97]$ \\
\hline 6 Mortality & 5 & 4829 & $\begin{array}{l}\text { Odds Ratio (M-H, } \\
\text { Fixed, } 95 \% \text { CI) }\end{array}$ & $0.79[0.65,0.97]$ \\
\hline $\begin{array}{l}\text { 6.1 Mortality: three year } \\
\text { data }\end{array}$ & 1 & 3057 & $\begin{array}{l}\text { Odds Ratio (M-H, } \\
\text { Fixed, } 95 \% \mathrm{CI})\end{array}$ & $0.81[0.66,0.99]$ \\
\hline $\begin{array}{l}6.2 \text { Mortality: >one and } \\
\text { <three year data }\end{array}$ & 0 & 0 & $\begin{array}{l}\text { Odds Ratio (M-H, } \\
\text { Fixed, } 95 \% \mathrm{CI})\end{array}$ & Not estimable \\
\hline 6.3 Mortality: one year data & 3 & 1426 & $\begin{array}{l}\text { Odds Ratio (M-H, } \\
\text { Fixed, } 95 \% \text { CI) }\end{array}$ & $0.63[0.21,1.90]$ \\
\hline 6.4 Mortality: 6 month data & 1 & 346 & $\begin{array}{l}\text { Odds Ratio (M-H, } \\
\text { Fixed, } 95 \% \mathrm{CI})\end{array}$ & $0.15[0.01,3.01]$ \\
\hline $\begin{array}{l}7 \text { Change from baseline in } \mathrm{St} \\
\text { George's Respiratory } \\
\text { Questionnaire (total score) }\end{array}$ & 4 & & $\begin{array}{l}\text { SGRQ units (Fixed, } \\
95 \% \mathrm{CI} \text { ) }\end{array}$ & $-2.90[-3.61,-2.18]$ \\
\hline $\begin{array}{l}\text { 7.1 Partially reversible } \\
\text { population (mixed population) }\end{array}$ & 0 & & $\begin{array}{l}\text { SGRQ units (Fixed, } \\
95 \% \mathrm{CI} \text { ) }\end{array}$ & Not estimable \\
\hline $\begin{array}{l}7.2 \text { Poorly reversible } \\
\text { population }\end{array}$ & 4 & & $\begin{array}{l}\text { SGRQ units (Fixed, } \\
95 \% \mathrm{CI} \text { ) }\end{array}$ & $-2.90[-3.61,-2.18]$ \\
\hline $\begin{array}{l}8 \text { Change from baseline in St } \\
\text { George's Respiratory } \\
\text { Questionnaire (domain - } \\
\text { symptoms) }\end{array}$ & 1 & & $\begin{array}{l}\text { SGRQ units (Fixed, } \\
95 \% \mathrm{CI} \text { ) }\end{array}$ & Totals not selected \\
\hline $\begin{array}{l}8.1 \text { Partially reversible } \\
\text { population (mixed population) }\end{array}$ & 0 & & $\begin{array}{l}\text { SGRQ units (Fixed, } \\
95 \% \mathrm{CI} \text { ) }\end{array}$ & Not estimable \\
\hline $\begin{array}{l}8.2 \text { Poorly reversible } \\
\text { population }\end{array}$ & 1 & & $\begin{array}{l}\text { SGRQ units (Fixed, } \\
95 \% \mathrm{CI} \text { ) }\end{array}$ & Not estimable \\
\hline $\begin{array}{l}9 \text { Change from baseline in St } \\
\text { George's Respiratory } \\
\text { Questionnaire (domain - } \\
\text { activity) }\end{array}$ & 1 & & $\begin{array}{l}\text { SGRQ units (Fixed, } \\
95 \% \text { CI) }\end{array}$ & Totals not selected \\
\hline $\begin{array}{l}9.1 \text { Partially reversible } \\
\text { population (mixed population) }\end{array}$ & 0 & & $\begin{array}{l}\text { SGRQ units (Fixed, } \\
\text { 95\% CI) }\end{array}$ & Not estimable \\
\hline $\begin{array}{l}9.2 \text { Poorly reversible } \\
\text { population }\end{array}$ & 1 & & $\begin{array}{l}\text { SGRQ units (Fixed, } \\
95 \% \mathrm{CI} \text { ) }\end{array}$ & Not estimable \\
\hline $\begin{array}{l}10 \text { Change from baseline in St } \\
\text { George's Respiratory } \\
\text { Questionnaire (domain - } \\
\text { impact) }\end{array}$ & 1 & & $\begin{array}{l}\text { SGRQ units (Fixed, } \\
95 \% \mathrm{CI} \text { ) }\end{array}$ & Totals not selected \\
\hline $\begin{array}{l}10.1 \text { Partially reversible } \\
\text { population (mixed population) }\end{array}$ & 0 & & $\begin{array}{l}\text { SGRQ units (Fixed, } \\
95 \% \mathrm{CI} \text { ) }\end{array}$ & Not estimable \\
\hline
\end{tabular}




\begin{tabular}{|c|c|c|c|c|}
\hline Outcome or subgroup title & No. of studies & No. of participants & Statistical method & Effect size \\
\hline $\begin{array}{l}10.2 \text { Poorly reversible } \\
\text { population }\end{array}$ & 1 & & $\begin{array}{l}\text { SGRQ units (Fixed, } \\
95 \% \mathrm{CI} \text { ) }\end{array}$ & Not estimable \\
\hline $\begin{array}{l}11 \text { End of treatment St } \\
\text { George's Respiratory } \\
\text { Questionnaire scores (total } \\
\text { score) }\end{array}$ & 1 & & $\begin{array}{l}\text { Mean Difference } \\
\text { (IV, Fixed, 95\% CI) }\end{array}$ & Totals not selected \\
\hline $\begin{array}{l}\text { 11.1 Partially reversible } \\
\text { population (mixed population) }\end{array}$ & 0 & & $\begin{array}{l}\text { Mean Difference } \\
\text { (IV, Fixed, 95\% CI) }\end{array}$ & Not estimable \\
\hline $\begin{array}{l}11.2 \text { Poorly reversible } \\
\text { population }\end{array}$ & 1 & & $\begin{array}{l}\text { Mean Difference } \\
\text { (IV, Fixed, 95\% CI) }\end{array}$ & Not estimable \\
\hline $\begin{array}{l}12 \text { End of treatment St } \\
\text { George's Respiratory } \\
\text { Questionnaire scores (domain } \\
\text { - breathlessness) }\end{array}$ & 1 & & $\begin{array}{l}\text { Mean Difference } \\
\text { (IV, Fixed, 95\% CI) }\end{array}$ & Totals not selected \\
\hline $\begin{array}{l}\text { 12.1 Partially reversible } \\
\text { population (mixed population) }\end{array}$ & 0 & & $\begin{array}{l}\text { Mean Difference } \\
\text { (IV, Fixed, 95\% CI) }\end{array}$ & Not estimable \\
\hline $\begin{array}{l}12.2 \text { Poorly reversible } \\
\text { population }\end{array}$ & 1 & & $\begin{array}{l}\text { Mean Difference } \\
\text { (IV, Fixed, 95\% CI) }\end{array}$ & Not estimable \\
\hline $\begin{array}{l}13 \text { Change from baseline in } \\
\text { Canadian Respiratory Disease } \\
\text { Questionnaire scores }\end{array}$ & 2 & 712 & $\begin{array}{l}\text { Mean Difference } \\
\text { (IV, Fixed, 95\% CI) }\end{array}$ & $5.0[2.48,7.52]$ \\
\hline $\begin{array}{l}\text { 13.1 Partially reversible } \\
\text { population (mixed population) }\end{array}$ & 2 & 712 & $\begin{array}{l}\text { Mean Difference } \\
\text { (IV, Fixed, 95\% CI) }\end{array}$ & $5.0[2.48,7.52]$ \\
\hline $\begin{array}{l}13.2 \text { Poorly reversible } \\
\text { population }\end{array}$ & 0 & 0 & $\begin{array}{l}\text { Mean Difference } \\
\text { (IV, Fixed, 95\% CI) }\end{array}$ & Not estimable \\
\hline $\begin{array}{l}14 \text { Change from baseline in } \\
\text { Transitional Dyspnoea Index } \\
\text { (TDI) scores }\end{array}$ & 2 & 707 & $\begin{array}{l}\text { Mean Difference } \\
\text { (IV, Fixed, 95\% CI) }\end{array}$ & $1.04[0.56,1.53]$ \\
\hline $\begin{array}{l}\text { 14.1 Partially reversible } \\
\text { population (mixed population) }\end{array}$ & 2 & 707 & $\begin{array}{l}\text { Mean Difference } \\
\text { (IV, Fixed, 95\% CI) }\end{array}$ & $1.04[0.56,1.53]$ \\
\hline $\begin{array}{l}14.2 \text { Poorly reversible } \\
\text { population }\end{array}$ & 0 & 0 & $\begin{array}{l}\text { Mean Difference } \\
\text { (IV, Fixed, 95\% CI) }\end{array}$ & Not estimable \\
\hline $\begin{array}{l}15 \text { End of treatment symptom } \\
\text { scores }\end{array}$ & 1 & & $\begin{array}{l}\text { Mean Difference } \\
\text { (IV, Fixed, 95\% CI) }\end{array}$ & Totals not selected \\
\hline $\begin{array}{l}15.1 \text { Partially reversible } \\
\text { population (mixed population) }\end{array}$ & 0 & & $\begin{array}{l}\text { Mean Difference } \\
\text { (IV, Fixed, 95\% CI) }\end{array}$ & Not estimable \\
\hline $\begin{array}{l}15.2 \text { Poorly reversible } \\
\text { population }\end{array}$ & 1 & & $\begin{array}{l}\text { Mean Difference } \\
\text { (IV, Fixed, 95\% CI) }\end{array}$ & Not estimable \\
\hline $\begin{array}{l}16 \text { Change from baseline in } \\
\text { predose FEV1 }\end{array}$ & 5 & & $\begin{array}{l}\text { Litres (Fixed, 95\% } \\
\text { CI) }\end{array}$ & $0.16[0.14,0.19]$ \\
\hline 16.1 Reversible population & 3 & & $\begin{array}{l}\text { Litres (Fixed, 95\% } \\
\text { CI) }\end{array}$ & $0.19[0.15,0.24]$ \\
\hline $\begin{array}{l}16.2 \text { Partially reversible } \\
\text { population (mixed population) }\end{array}$ & 0 & & $\begin{array}{l}\text { Litres (Fixed, 95\% } \\
\text { CI) }\end{array}$ & Not estimable \\
\hline $\begin{array}{l}16.3 \text { Poorly reversible } \\
\text { population }\end{array}$ & 4 & & $\begin{array}{l}\text { Litres (Fixed, 95\% } \\
\text { CI) }\end{array}$ & $0.15[0.11,0.18]$ \\
\hline 16.4 Unclear reversibility & 0 & & $\begin{array}{l}\text { Litres (Fixed, 95\% } \\
\text { CI) }\end{array}$ & Not estimable \\
\hline $\begin{array}{l}17 \text { Change from baseline in } \\
\text { postdose FEV1 }\end{array}$ & 1 & & $\begin{array}{l}\text { Litres (Fixed, 95\% } \\
\text { CI) }\end{array}$ & Totals not selected \\
\hline 17.1 Reversible population & 0 & & $\begin{array}{l}\text { Litres (Fixed, 95\% } \\
\text { CI) }\end{array}$ & Not estimable \\
\hline $\begin{array}{l}\text { 17.2 Partially reversible } \\
\text { population (mixed population) }\end{array}$ & 0 & & $\begin{array}{l}\text { Litres (Fixed, 95\% } \\
\text { CI) }\end{array}$ & Not estimable \\
\hline
\end{tabular}




\begin{tabular}{|c|c|c|c|c|}
\hline Outcome or subgroup title & No. of studies & No. of participants & Statistical method & Effect size \\
\hline $\begin{array}{l}17.3 \text { Poorly reversible } \\
\text { population }\end{array}$ & 1 & & $\begin{array}{l}\text { Litres (Fixed, 95\% } \\
\text { CI) }\end{array}$ & Not estimable \\
\hline 17.4 Unclear reversibility & 0 & & $\begin{array}{l}\text { Litres (Fixed, 95\% } \\
\text { CI) }\end{array}$ & Not estimable \\
\hline $\begin{array}{l}18 \text { End of treatment FEV1 (\% } \\
\text { predicted) }\end{array}$ & 1 & & $\begin{array}{l}\text { Std. Mean } \\
\text { Difference (IV, } \\
\text { Fixed, } 95 \% \mathrm{CI})\end{array}$ & Totals not selected \\
\hline $\begin{array}{l}18.1 \text { Partially reversible } \\
\text { population (mixed population) }\end{array}$ & 0 & & $\begin{array}{l}\text { Std. Mean } \\
\text { Difference (IV, } \\
\text { Fixed, } 95 \% \mathrm{CI})\end{array}$ & Not estimable \\
\hline $\begin{array}{l}18.2 \text { Poorly reversible } \\
\text { population }\end{array}$ & 1 & & $\begin{array}{l}\text { Std. Mean } \\
\text { Difference (IV, } \\
\text { Fixed, } 95 \% \mathrm{CI})\end{array}$ & Not estimable \\
\hline $\begin{array}{l}19 \text { End of treatment FEV1 } \\
\text { (Litres) }\end{array}$ & 2 & & $\begin{array}{l}\text { Litres (Fixed, 95\% } \\
\text { CI) }\end{array}$ & $0.13[0.10,0.16]$ \\
\hline $\begin{array}{l}19.1 \text { Partially reversible } \\
\text { population (mixed population) }\end{array}$ & 0 & & $\begin{array}{l}\text { Litres (Fixed, 95\% } \\
\text { CI) }\end{array}$ & Not estimable \\
\hline $\begin{array}{l}19.2 \text { Poorly reversible } \\
\text { population }\end{array}$ & 2 & & $\begin{array}{l}\text { Litres (Fixed, 95\% } \\
\text { CI) }\end{array}$ & $0.13[0.10,0.16]$ \\
\hline $\begin{array}{l}20 \text { End of treatment am PEF } \\
(\mathrm{L} / \mathrm{min})\end{array}$ & 1 & & $\begin{array}{l}\mathrm{L} / \min \text { (Fixed, } 95 \% \\
\mathrm{CI} \text { ) }\end{array}$ & Totals not selected \\
\hline $\begin{array}{l}20.1 \text { Partially reversible } \\
\text { population (mixed population) }\end{array}$ & 0 & & $\begin{array}{l}\mathrm{L} / \mathrm{min} \text { (Fixed, } 95 \% \\
\mathrm{CI} \text { ) }\end{array}$ & Not estimable \\
\hline $\begin{array}{l}20.2 \text { Poorly reversible } \\
\text { population }\end{array}$ & 1 & & $\begin{array}{l}\mathrm{L} / \min \text { (Fixed, } 95 \% \\
\mathrm{CI} \text { ) }\end{array}$ & Not estimable \\
\hline $\begin{array}{l}21 \text { End of treatment shuttle } \\
\text { walk test }\end{array}$ & 1 & & $\begin{array}{l}\text { Metres (Fixed, 95\% } \\
\text { CI) }\end{array}$ & Totals not selected \\
\hline $\begin{array}{c}21.1 \text { Partially reversible } \\
\text { population (mixed population) }\end{array}$ & 0 & & $\begin{array}{l}\text { Metres (Fixed, 95\% } \\
\text { CI) }\end{array}$ & Not estimable \\
\hline $\begin{array}{l}21.2 \text { Poorly reversible } \\
\text { population }\end{array}$ & 1 & & $\begin{array}{l}\text { Metres (Fixed, 95\% } \\
\text { CI) }\end{array}$ & Not estimable \\
\hline $\begin{array}{l}22 \text { End of treatment rescue } \\
\text { medication usage (puffs/day) }\end{array}$ & 1 & & $\begin{array}{l}\text { Mean Difference } \\
\text { (IV, Fixed, 95\% CI) }\end{array}$ & Totals not selected \\
\hline $\begin{array}{l}22.1 \text { Partially reversible } \\
\text { population (mixed population) }\end{array}$ & 0 & & $\begin{array}{l}\text { Mean Difference } \\
\text { (IV, Fixed, 95\% CI) }\end{array}$ & Not estimable \\
\hline $\begin{array}{l}22.2 \text { Poorly reversible } \\
\text { population }\end{array}$ & 1 & & $\begin{array}{l}\text { Mean Difference } \\
\text { (IV, Fixed, 95\% CI) }\end{array}$ & Not estimable \\
\hline $\begin{array}{l}23 \text { Change from baseline in } \\
\text { rescue medication usage } \\
\text { (puffs/day) }\end{array}$ & 2 & 703 & $\begin{array}{l}\text { Mean Difference } \\
\text { (IV, Fixed, 95\% CI) }\end{array}$ & $-1.19[-1.83,-0.55]$ \\
\hline $\begin{array}{l}23.1 \text { Partially reversible } \\
\text { population (mixed population) }\end{array}$ & 2 & 703 & $\begin{array}{l}\text { Mean Difference } \\
\text { (IV, Fixed, 95\% CI) }\end{array}$ & $-1.19[-1.83,-0.55]$ \\
\hline $\begin{array}{l}23.2 \text { Poorly reversible } \\
\text { population }\end{array}$ & 0 & 0 & $\begin{array}{l}\text { Mean Difference } \\
\text { (IV, Fixed, 95\% CI) }\end{array}$ & Not estimable \\
\hline 24 Adverse events - any event & 8 & 5493 & $\begin{array}{l}\text { Odds Ratio (M-H, } \\
\text { Fixed, } 95 \% \mathrm{CI})\end{array}$ & $1.10[0.96,1.27]$ \\
\hline 24.1 Reversible population & 1 & 126 & $\begin{array}{l}\text { Odds Ratio (M-H, } \\
\text { Fixed, } 95 \% \mathrm{CI})\end{array}$ & $1.20[0.59,2.46]$ \\
\hline $\begin{array}{l}24.2 \text { Partially reversible } \\
\text { population (mixed population) }\end{array}$ & 2 & 717 & $\begin{array}{l}\text { Odds Ratio (M-H, } \\
\text { Fixed, } 95 \% \text { CI) }\end{array}$ & $1.42[1.03,1.96]$ \\
\hline $\begin{array}{l}24.3 \text { Poorly reversible } \\
\text { population }\end{array}$ & 5 & 4650 & $\begin{array}{l}\text { Odds Ratio (M-H, } \\
\text { Fixed, } 95 \% \text { CI) }\end{array}$ & $1.03[0.88,1.21]$ \\
\hline $\begin{array}{l}25 \text { Adverse events - } \\
\text { candidiasis }\end{array}$ & 6 & 1958 & $\begin{array}{l}\text { Odds Ratio (M-H, } \\
\text { Fixed, } 95 \% \text { CI) }\end{array}$ & $5.73[3.07,10.67]$ \\
\hline
\end{tabular}




\begin{tabular}{|c|c|c|c|c|}
\hline Outcome or subgroup title & No. of studies & No. of participants & Statistical method & Effect size \\
\hline 25.1 Reversible population & 1 & 126 & $\begin{array}{l}\text { Odds Ratio (M-H, } \\
\text { Fixed, } 95 \% \text { CI) }\end{array}$ & $1.03[0.06,16.88]$ \\
\hline $\begin{array}{l}\text { 25.2 Partially reversible } \\
\text { population (mixed population) }\end{array}$ & 2 & 717 & $\begin{array}{l}\text { Odds Ratio }(\mathrm{M}-\mathrm{H}, \\
\text { Fixed, } 95 \% \mathrm{CI})\end{array}$ & $11.13[3.36,36.90]$ \\
\hline $\begin{array}{l}25.3 \text { Poorly reversible } \\
\text { population }\end{array}$ & 3 & 1115 & $\begin{array}{l}\text { Odds Ratio (M-H, } \\
\text { Fixed, } 95 \% \mathrm{CI})\end{array}$ & $4.40[2.01,9.62]$ \\
\hline 25.4 Unclear reversibility & 0 & 0 & $\begin{array}{l}\text { Odds Ratio (M-H, } \\
\text { Fixed, } 95 \% \mathrm{CI})\end{array}$ & Not estimable \\
\hline $\begin{array}{l}26 \text { Withdrawals due to adverse } \\
\text { events }\end{array}$ & 8 & 5110 & $\begin{array}{l}\text { Odds Ratio (M-H, } \\
\text { Fixed, } 95 \% \text { CI) }\end{array}$ & $0.76[0.65,0.88]$ \\
\hline $\begin{array}{l}\text { 26.1 Partially reversible } \\
\text { population (mixed population) }\end{array}$ & 1 & 354 & $\begin{array}{l}\text { Odds Ratio (M-H, } \\
\text { Fixed, } 95 \% \text { CI) }\end{array}$ & $0.69[0.31,1.51]$ \\
\hline $\begin{array}{l}26.2 \text { Poorly reversible } \\
\text { population }\end{array}$ & 6 & 4630 & $\begin{array}{l}\text { Odds Ratio (M-H, } \\
\text { Fixed, } 95 \% \text { CI) }\end{array}$ & $0.76[0.65,0.89]$ \\
\hline 26.3 Unclear reversibility & 1 & 126 & $\begin{array}{l}\text { Odds Ratio (M-H, } \\
\text { Fixed, } 95 \% \mathrm{CI})\end{array}$ & $0.34[0.01,8.47]$ \\
\hline $\begin{array}{l}27 \text { Withdrawals due to lack of } \\
\text { efficacy/exacerbations }\end{array}$ & 5 & 4620 & $\begin{array}{l}\text { Odds Ratio (M-H, } \\
\text { Fixed, } 95 \% \mathrm{CI})\end{array}$ & $0.30[0.21,0.42]$ \\
\hline $\begin{array}{l}\text { 27.1 Partially reversible } \\
\text { population (mixed population) }\end{array}$ & 0 & 0 & $\begin{array}{l}\text { Odds Ratio (M-H, } \\
\text { Fixed, } 95 \% \text { CI) }\end{array}$ & Not estimable \\
\hline $\begin{array}{l}27.2 \text { Poorly reversible } \\
\text { population }\end{array}$ & 5 & 4620 & $\begin{array}{l}\text { Odds Ratio (M-H, } \\
\text { Fixed, } 95 \% \text { CI) }\end{array}$ & $0.30[0.21,0.42]$ \\
\hline 27.3 Unclear reversibility & 0 & 0 & $\begin{array}{l}\text { Odds Ratio (M-H, } \\
\text { Fixed, } 95 \% \text { CI) }\end{array}$ & Not estimable \\
\hline 28 Withdrawals & 8 & 5450 & $\begin{array}{l}\text { Odds Ratio (M-H, } \\
\text { Fixed, } 95 \% \mathrm{CI})\end{array}$ & $0.70[0.62,0.78]$ \\
\hline 28.1 Reversible population & 1 & 121 & $\begin{array}{l}\text { Odds Ratio (M-H, } \\
\text { Fixed, } 95 \% \text { CI) }\end{array}$ & $2.95[0.30,29.18]$ \\
\hline $\begin{array}{l}28.2 \text { Partially reversible } \\
\text { population (mixed population) }\end{array}$ & 2 & 709 & $\begin{array}{l}\text { Odds Ratio (M-H, } \\
\text { Fixed, } 95 \% \mathrm{CI})\end{array}$ & $0.82[0.60,1.13]$ \\
\hline $\begin{array}{l}28.3 \text { Poorly reversible } \\
\text { population }\end{array}$ & 5 & 4620 & $\begin{array}{l}\text { Odds Ratio (M-H, } \\
\text { Fixed, } 95 \% \mathrm{CI})\end{array}$ & $0.68[0.60,0.76]$ \\
\hline 28.4 Unclear reversibility & 0 & 0 & $\begin{array}{l}\text { Odds Ratio (M-H, } \\
\text { Fixed, } 95 \% \mathrm{CI})\end{array}$ & Not estimable \\
\hline $\begin{array}{l}29 \text { Adverse events - } \\
\text { pneumonia }\end{array}$ & 7 & 5229 & $\begin{array}{l}\text { Odds Ratio (M-H, } \\
\text { Fixed, } 95 \% \mathrm{CI})\end{array}$ & $1.80[1.48,2.18]$ \\
\hline 29.1 Reversible population & 1 & 126 & $\begin{array}{l}\text { Odds Ratio }(\mathrm{M}-\mathrm{H}, \\
\text { Fixed, } 95 \% \mathrm{CI})\end{array}$ & $0.34[0.01,8.47]$ \\
\hline $\begin{array}{l}\text { 29.2 Partially reversible } \\
\text { population (mixed population) }\end{array}$ & 2 & 709 & $\begin{array}{l}\text { Odds Ratio (M-H, } \\
\text { Fixed, } 95 \% \text { CI) }\end{array}$ & $5.55[0.26,116.46]$ \\
\hline $\begin{array}{l}29.3 \text { Poorly reversible } \\
\text { population }\end{array}$ & 4 & 4394 & $\begin{array}{l}\text { Odds Ratio (M-H, } \\
\text { Fixed, } 95 \% \mathrm{CI})\end{array}$ & $1.80[1.48,2.18]$ \\
\hline 29.4 Unclear reversibility & 0 & 0 & $\begin{array}{l}\text { Odds Ratio (M-H, } \\
\text { Fixed, } 95 \% \mathrm{CI})\end{array}$ & Not estimable \\
\hline $\begin{array}{l}30 \text { Adverse events - } \\
\text { nasopharyngitis }\end{array}$ & 2 & 3535 & $\begin{array}{l}\text { Odds Ratio (M-H, } \\
\text { Fixed, } 95 \% \mathrm{CI})\end{array}$ & $1.28[1.05,1.56]$ \\
\hline $\begin{array}{l}\text { 30.1 Partially reversible } \\
\text { population (mixed population) }\end{array}$ & 0 & 0 & $\begin{array}{l}\text { Odds Ratio (M-H, } \\
\text { Fixed, } 95 \% \text { CI) }\end{array}$ & Not estimable \\
\hline $\begin{array}{l}30.2 \text { Poorly reversible } \\
\text { population }\end{array}$ & 2 & 3535 & $\begin{array}{l}\text { Odds Ratio (M-H, } \\
\text { Fixed, } 95 \% \mathrm{CI})\end{array}$ & $1.28[1.05,1.56]$ \\
\hline 30.3 Unclear reversibility & 0 & 0 & $\begin{array}{l}\text { Odds Ratio (M-H, } \\
\text { Fixed, } 95 \% \mathrm{CI})\end{array}$ & Not estimable \\
\hline $\begin{array}{l}31 \text { Adverse events - } \\
\text { pharyngolaryngeal pain }\end{array}$ & 1 & & $\begin{array}{l}\text { Odds Ratio (M-H, } \\
\text { Fixed, } 95 \% \mathrm{CI})\end{array}$ & Totals not selected \\
\hline
\end{tabular}




\begin{tabular}{|c|c|c|c|c|}
\hline Outcome or subgroup title & No. of studies & No. of participants & Statistical method & Effect size \\
\hline $\begin{array}{l}\text { 31.1 Partially reversible } \\
\text { population (mixed population) }\end{array}$ & 0 & & $\begin{array}{l}\text { Odds Ratio (M-H, } \\
\text { Fixed, } 95 \% \text { CI) }\end{array}$ & Not estimable \\
\hline $\begin{array}{l}31.2 \text { Poorly reversible } \\
\text { population }\end{array}$ & 1 & & $\begin{array}{l}\text { Odds Ratio (M-H, } \\
\text { Fixed, } 95 \% \mathrm{CI})\end{array}$ & Not estimable \\
\hline 31.3 Unclear reversibility & 0 & & $\begin{array}{l}\text { Odds Ratio (M-H, } \\
\text { Fixed, } 95 \% \mathrm{CI})\end{array}$ & Not estimable \\
\hline $\begin{array}{l}32 \text { Adverse events - upper } \\
\text { respiratory tract infection }\end{array}$ & 5 & 4963 & $\begin{array}{l}\text { Odds Ratio (M-H, } \\
\text { Fixed, } 95 \% \mathrm{CI})\end{array}$ & $1.23[1.04,1.47]$ \\
\hline $\begin{array}{l}32.1 \text { Partially reversible } \\
\text { population (mixed population) }\end{array}$ & 2 & 709 & $\begin{array}{l}\text { Odds Ratio (M-H, } \\
\text { Fixed, } 95 \% \mathrm{CI})\end{array}$ & $1.25[0.81,1.92]$ \\
\hline $\begin{array}{l}32.2 \text { Poorly reversible } \\
\text { population }\end{array}$ & 3 & 4254 & $\begin{array}{l}\text { Odds Ratio (M-H, } \\
\text { Fixed, } 95 \% \mathrm{CI})\end{array}$ & $1.23[1.02,1.48]$ \\
\hline 32.3 Unclear reversibility & 0 & 0 & $\begin{array}{l}\text { Odds Ratio (M-H, } \\
\text { Fixed, } 95 \% \mathrm{CI})\end{array}$ & Not estimable \\
\hline 33 Adverse events - headache & 5 & 4367 & $\begin{array}{l}\text { Odds Ratio (M-H, } \\
\text { Fixed, } 95 \% \mathrm{CI})\end{array}$ & $1.06[0.85,1.32]$ \\
\hline 33.1 Reversible population & 1 & 123 & $\begin{array}{l}\text { Odds Ratio (M-H, } \\
\text { Fixed, } 95 \% \mathrm{CI})\end{array}$ & $0.22[0.02,2.01]$ \\
\hline $\begin{array}{l}\text { 33.2 Partially reversible } \\
\text { population (mixed population) }\end{array}$ & 2 & 709 & $\begin{array}{l}\text { Odds Ratio (M-H, } \\
\text { Fixed, } 95 \% \mathrm{CI})\end{array}$ & $1.38[0.91,2.10]$ \\
\hline $\begin{array}{l}33.3 \text { Poorly reversible } \\
\text { population }\end{array}$ & 2 & 3535 & $\begin{array}{l}\text { Odds Ratio (M-H, } \\
\text { Fixed, } 95 \% \mathrm{CI})\end{array}$ & $0.97[0.75,1.27]$ \\
\hline 33.4 Unclear reversibility & 0 & 0 & $\begin{array}{l}\text { Odds Ratio (M-H, } \\
\text { Fixed, } 95 \% \text { CI) }\end{array}$ & Not estimable \\
\hline $\begin{array}{l}34 \text { Adverse events - } \\
\text { hoarseness }\end{array}$ & 2 & 585 & $\begin{array}{l}\text { Odds Ratio (M-H, } \\
\text { Fixed, } 95 \% \mathrm{CI})\end{array}$ & $8.79[1.11,69.62]$ \\
\hline $\begin{array}{l}34.1 \text { Partially reversible } \\
\text { population (mixed population) }\end{array}$ & 0 & 0 & $\begin{array}{l}\text { Odds Ratio }(\mathrm{M}-\mathrm{H}, \\
\text { Fixed, } 95 \% \mathrm{CI})\end{array}$ & Not estimable \\
\hline $\begin{array}{l}34.2 \text { Poorly reversible } \\
\text { population }\end{array}$ & 2 & 585 & $\begin{array}{l}\text { Odds Ratio (M-H, } \\
\text { Fixed, } 95 \% \mathrm{CI})\end{array}$ & $8.79[1.11,69.62]$ \\
\hline 34.3 Unclear reversibility & 0 & 0 & $\begin{array}{l}\text { Odds Ratio (M-H, } \\
\text { Fixed, } 95 \% \mathrm{CI})\end{array}$ & Not estimable \\
\hline 35 Adverse events - pyrexia & 1 & & $\begin{array}{l}\text { Odds Ratio (M-H, } \\
\text { Fixed, } 95 \% \mathrm{CI})\end{array}$ & Totals not selected \\
\hline $\begin{array}{l}\text { 35.1 Partially reversible } \\
\text { population (mixed population) }\end{array}$ & 0 & & $\begin{array}{l}\text { Odds Ratio (M-H, } \\
\text { Fixed, } 95 \% \mathrm{CI})\end{array}$ & Not estimable \\
\hline $\begin{array}{l}35.2 \text { Poorly reversible } \\
\text { population }\end{array}$ & 1 & & $\begin{array}{l}\text { Odds Ratio (M-H, } \\
\text { Fixed, } 95 \% \text { CI) }\end{array}$ & Not estimable \\
\hline 35.3 Unclear reversibility & 0 & & $\begin{array}{l}\text { Odds Ratio (M-H, } \\
\text { Fixed, } 95 \% \mathrm{CI})\end{array}$ & Not estimable \\
\hline 36 Adverse events - cough & 4 & 1057 & $\begin{array}{l}\text { Odds Ratio (M-H, } \\
\text { Fixed, } 95 \% \mathrm{CI})\end{array}$ & $0.61[0.29,1.29]$ \\
\hline 36.1 Reversible population & 1 & 126 & $\begin{array}{l}\text { Odds Ratio }(\mathrm{M}-\mathrm{H}, \\
\text { Fixed, } 95 \% \mathrm{CI})\end{array}$ & $3.15[0.13,78.72]$ \\
\hline $\begin{array}{l}\text { 36.2 Partially reversible } \\
\text { population (mixed population) }\end{array}$ & 1 & 346 & $\begin{array}{l}\text { Odds Ratio (M-H, } \\
\text { Fixed, } 95 \% \mathrm{CI})\end{array}$ & $0.49[0.18,1.31]$ \\
\hline $\begin{array}{l}36.3 \text { Poorly reversible } \\
\text { population }\end{array}$ & 2 & 585 & $\begin{array}{l}\text { Odds Ratio (M-H, } \\
\text { Fixed, } 95 \% \mathrm{CI})\end{array}$ & $0.66[0.18,2.44]$ \\
\hline 36.4 Unclear reversibility & 0 & 0 & $\begin{array}{l}\text { Odds Ratio (M-H, } \\
\text { Fixed, } 95 \% \mathrm{CI})\end{array}$ & Not estimable \\
\hline $\begin{array}{l}37 \text { Adverse events - } \\
\text { palpitations }\end{array}$ & 1 & & $\begin{array}{l}\text { Odds Ratio (M-H, } \\
\text { Fixed, } 95 \% \text { CI) }\end{array}$ & Totals not selected \\
\hline $\begin{array}{l}\text { 37.1 Partially reversible } \\
\text { population (mixed population) }\end{array}$ & 0 & & $\begin{array}{l}\text { Odds Ratio (M-H, } \\
\text { Fixed, } 95 \% \mathrm{CI})\end{array}$ & Not estimable \\
\hline
\end{tabular}




\begin{tabular}{|c|c|c|c|c|}
\hline Outcome or subgroup title & No. of studies & No. of participants & Statistical method & Effect size \\
\hline $\begin{array}{l}37.2 \text { Poorly reversible } \\
\text { population }\end{array}$ & 1 & & $\begin{array}{l}\text { Odds Ratio (M-H, } \\
\text { Fixed, } 95 \% \text { CI) }\end{array}$ & Not estimable \\
\hline 37.3 Unclear reversibility & 0 & & $\begin{array}{l}\text { Odds Ratio (M-H, } \\
\text { Fixed, } 95 \% \mathrm{CI})\end{array}$ & Not estimable \\
\hline $\begin{array}{l}38 \text { Adverse events - mouth } \\
\text { ulceration }\end{array}$ & 1 & & $\begin{array}{l}\text { Odds Ratio (M-H, } \\
\text { Fixed, } 95 \% \mathrm{CI})\end{array}$ & Totals not selected \\
\hline $\begin{array}{l}\text { 38.1 Partially reversible } \\
\text { population (mixed population) }\end{array}$ & 0 & & $\begin{array}{l}\text { Odds Ratio (M-H, } \\
\text { Fixed, } 95 \% \mathrm{CI})\end{array}$ & Not estimable \\
\hline $\begin{array}{l}38.2 \text { Poorly reversible } \\
\text { population }\end{array}$ & 1 & & $\begin{array}{l}\text { Odds Ratio (M-H, } \\
\text { Fixed, } 95 \% \mathrm{CI})\end{array}$ & Not estimable \\
\hline 38.3 Unclear reversibility & 0 & & $\begin{array}{l}\text { Odds Ratio (M-H, } \\
\text { Fixed, } 95 \% \mathrm{CI})\end{array}$ & Not estimable \\
\hline 39 Adverse events - toothache & 1 & & $\begin{array}{l}\text { Odds Ratio (M-H, } \\
\text { Fixed, } 95 \% \mathrm{CI})\end{array}$ & Totals not selected \\
\hline $\begin{array}{l}39.1 \text { Partially reversible } \\
\text { population (mixed population) }\end{array}$ & 0 & & $\begin{array}{l}\text { Odds Ratio (M-H, } \\
\text { Fixed, } 95 \% \mathrm{CI})\end{array}$ & Not estimable \\
\hline $\begin{array}{l}39.2 \text { Poorly reversible } \\
\text { population }\end{array}$ & 1 & & $\begin{array}{l}\text { Odds Ratio (M-H, } \\
\text { Fixed, } 95 \% \mathrm{CI})\end{array}$ & Not estimable \\
\hline 39.3 Unclear reversibility & 0 & & $\begin{array}{l}\text { Odds Ratio (M-H, } \\
\text { Fixed, } 95 \% \mathrm{CI})\end{array}$ & Not estimable \\
\hline $\begin{array}{l}40 \text { Adverse events - urinary } \\
\text { tract infection }\end{array}$ & 1 & & $\begin{array}{l}\text { Odds Ratio (M-H, } \\
\text { Fixed, } 95 \% \mathrm{CI})\end{array}$ & Totals not selected \\
\hline $\begin{array}{l}\text { 40.1 Partially reversible } \\
\text { population (mixed population) }\end{array}$ & 0 & & $\begin{array}{l}\text { Odds Ratio (M-H, } \\
\text { Fixed, } 95 \% \mathrm{CI})\end{array}$ & Not estimable \\
\hline $\begin{array}{l}40.2 \text { Poorly reversible } \\
\text { population }\end{array}$ & 1 & & $\begin{array}{l}\text { Odds Ratio (M-H, } \\
\text { Fixed, } 95 \% \mathrm{CI})\end{array}$ & Not estimable \\
\hline 40.3 Unclear reversibility & 0 & & $\begin{array}{l}\text { Odds Ratio }(\mathrm{M}-\mathrm{H}, \\
\text { Fixed, } 95 \% \mathrm{CI})\end{array}$ & Not estimable \\
\hline 41 Adverse events - dyspnoea & 1 & & $\begin{array}{l}\text { Odds Ratio (M-H, } \\
\text { Fixed, } 95 \% \mathrm{CI})\end{array}$ & Totals not selected \\
\hline $\begin{array}{l}41.1 \text { Partially reversible } \\
\text { population (mixed population) }\end{array}$ & 0 & & $\begin{array}{l}\text { Odds Ratio (M-H, } \\
\text { Fixed, } 95 \% \mathrm{CI})\end{array}$ & Not estimable \\
\hline $\begin{array}{l}41.2 \text { Poorly reversible } \\
\text { population }\end{array}$ & 1 & & $\begin{array}{l}\text { Odds Ratio (M-H, } \\
\text { Fixed, } 95 \% \mathrm{CI})\end{array}$ & Not estimable \\
\hline 41.3 Unclear reversibility & 0 & & $\begin{array}{l}\text { Odds Ratio (M-H, } \\
\text { Fixed, } 95 \% \mathrm{CI})\end{array}$ & Not estimable \\
\hline $\begin{array}{l}42 \text { Adverse events - blood } \\
\text { glucose increased }\end{array}$ & 1 & & $\begin{array}{l}\text { Odds Ratio (M-H, } \\
\text { Fixed, } 95 \% \mathrm{CI})\end{array}$ & Totals not selected \\
\hline $\begin{array}{l}\text { 42.1 Partially reversible } \\
\text { population (mixed population) }\end{array}$ & 0 & & $\begin{array}{l}\text { Odds Ratio (M-H, } \\
\text { Fixed, } 95 \% \mathrm{CI})\end{array}$ & Not estimable \\
\hline $\begin{array}{l}42.2 \text { Poorly reversible } \\
\text { population }\end{array}$ & 1 & & $\begin{array}{l}\text { Odds Ratio (M-H, } \\
\text { Fixed, } 95 \% \mathrm{CI})\end{array}$ & Not estimable \\
\hline 42.3 Unclear reversibility & 0 & & $\begin{array}{l}\text { Odds Ratio (M-H, } \\
\text { Fixed, } 95 \% \mathrm{CI})\end{array}$ & Not estimable \\
\hline 43 Adverse events - insomnia & 1 & & $\begin{array}{l}\text { Odds Ratio (M-H, } \\
\text { Fixed, } 95 \% \mathrm{CI})\end{array}$ & Totals not selected \\
\hline $\begin{array}{l}\text { 43.1 Partially reversible } \\
\text { population (mixed population) }\end{array}$ & 0 & & $\begin{array}{l}\text { Odds Ratio (M-H, } \\
\text { Fixed, } 95 \% \mathrm{CI})\end{array}$ & Not estimable \\
\hline $\begin{array}{l}43.2 \text { Poorly reversible } \\
\text { population }\end{array}$ & 1 & & $\begin{array}{l}\text { Odds Ratio (M-H, } \\
\text { Fixed, } 95 \% \mathrm{CI})\end{array}$ & Not estimable \\
\hline 43.3 Unclear reversibility & 0 & & $\begin{array}{l}\text { Odds Ratio (M-H, } \\
\text { Fixed, } 95 \% \mathrm{CI})\end{array}$ & Not estimable \\
\hline 44 Adverse events - bronchitis & 1 & & $\begin{array}{l}\text { Odds Ratio (M-H, } \\
\text { Fixed, } 95 \% \text { CI) }\end{array}$ & Totals not selected \\
\hline
\end{tabular}




\begin{tabular}{ccccc}
\hline Outcome or subgroup title & No. of studies & No. of participants & Statistical method & Effect size \\
\hline $\begin{array}{c}44.1 \text { Partially reversible } \\
\text { population (mixed population) }\end{array}$ & 0 & $\begin{array}{l}\text { Odds Ratio (M-H, } \\
\text { Fixed, 95\% CI) }\end{array}$ & Not estimable \\
$\begin{array}{c}44.2 \text { Poorly reversible } \\
\text { population }\end{array}$ & 1 & Odds Ratio (M-H, & Not estimable \\
44.3 Unclear reversibility & 0 & Fixed, 95\% CI) & Not estimable \\
\end{tabular}

\section{Comparison 3}

Budesonide/formoterol (BDF) versus placebo (PLA)

\begin{tabular}{|c|c|c|c|c|}
\hline Outcome or subgroup title & No. of studies & No. of participants & Statistical method & Effect size \\
\hline 1 Severe Exacerbations & 2 & & $\begin{array}{l}\text { Rate ratio (Fixed, 95\% } \\
\text { CI) }\end{array}$ & $0.74[0.62,0.88]$ \\
\hline 1.1 Partially reversible & 0 & & $\begin{array}{l}\text { Rate ratio (Fixed, 95\% } \\
\text { CI) }\end{array}$ & Not estimable \\
\hline 1.2 Poorly reversible & 2 & & $\begin{array}{l}\text { Rate ratio (Fixed, 95\% } \\
\text { CI) }\end{array}$ & $0.74[0.62,0.88]$ \\
\hline $\begin{array}{l}2 \text { Mean severe exacerbation } \\
\text { rates per patient per year }\end{array}$ & 2 & & $\begin{array}{l}\text { Mean Difference (IV, } \\
\text { Fixed, 95\% CI) }\end{array}$ & Totals not selected \\
\hline $\begin{array}{l}2.1 \text { Partially reversible } \\
\text { population (mixed } \\
\text { population) }\end{array}$ & 0 & & $\begin{array}{l}\text { Mean Difference (IV, } \\
\text { Fixed, 95\% CI) }\end{array}$ & Not estimable \\
\hline $\begin{array}{l}2.2 \text { Poorly reversible } \\
\text { population }\end{array}$ & 2 & & $\begin{array}{l}\text { Mean Difference (IV, } \\
\text { Fixed, 95\% CI) }\end{array}$ & Not estimable \\
\hline $\begin{array}{l}3 \text { Quality of life - SGRQ } \\
\text { (change scores) }\end{array}$ & 2 & & $\begin{array}{l}\text { Mean Difference (IV, } \\
\text { Fixed, 95\% CI) }\end{array}$ & Totals not selected \\
\hline $\begin{array}{l}\text { 3.1 Partially reversible } \\
\text { population (mixed } \\
\text { population) }\end{array}$ & 0 & & $\begin{array}{l}\text { Mean Difference (IV, } \\
\text { Fixed, 95\% CI) }\end{array}$ & Not estimable \\
\hline $\begin{array}{l}3.2 \text { Poorly reversible } \\
\text { population }\end{array}$ & 2 & & $\begin{array}{l}\text { Mean Difference (IV, } \\
\text { Fixed, 95\% CI) }\end{array}$ & Not estimable \\
\hline $\begin{array}{l}4 \text { Quality of life - change } \\
\text { scores }\end{array}$ & 2 & & $\begin{array}{l}\text { SGRQ (Fixed, 95\% } \\
\text { CI) }\end{array}$ & $-6.06[-7.90,-4.22]$ \\
\hline $\begin{array}{l}\text { 4.1 Partially reversible } \\
\text { (mixed population) }\end{array}$ & 0 & & $\begin{array}{l}\text { SGRQ (Fixed, 95\% } \\
\text { CI) }\end{array}$ & Not estimable \\
\hline 4.2 Poorly reversible & 2 & & $\begin{array}{l}\text { SGRQ (Fixed, } 95 \% \\
\text { CI) }\end{array}$ & $-6.06[-7.90,-4.22]$ \\
\hline 5 Rescue medication usage & 2 & & $\begin{array}{l}\text { Puffs per day (Fixed, } \\
95 \% \text { CI) }\end{array}$ & $-0.87[-1.15,-0.58]$ \\
\hline $\begin{array}{l}5.1 \text { Partially reversible } \\
\text { (mixed population) }\end{array}$ & 0 & & $\begin{array}{l}\text { Puffs per day (Fixed, } \\
95 \% \text { CI) }\end{array}$ & Not estimable \\
\hline 5.2 Poorly reversible & 2 & & $\begin{array}{l}\text { Puffs per day (Fixed, } \\
95 \% \text { CI) }\end{array}$ & $-0.87[-1.15,-0.58]$ \\
\hline $\begin{array}{l}6 \text { Symptoms (change } \\
\text { scores) }\end{array}$ & 2 & & $\begin{array}{l}\text { Symptom scale (Fixed, } \\
95 \% \text { CI) }\end{array}$ & $-0.63[-0.90,-0.37]$ \\
\hline $\begin{array}{l}\text { 6.1 Partially reversible } \\
\text { (mixed population) }\end{array}$ & 0 & & $\begin{array}{l}\text { Symptom scale (Fixed, } \\
95 \% \text { CI) }\end{array}$ & Not estimable \\
\hline 6.2 Poorly reversible & 2 & & $\begin{array}{l}\text { Symptom scale (Fixed, } \\
95 \% \text { CI) }\end{array}$ & $-0.63[-0.90,-0.37]$ \\
\hline $\begin{array}{l}7 \text { Mean FEV1 ( } \% \text { change } \\
\text { from baseline) }\end{array}$ & 2 & & $\begin{array}{l}\% \text { increase (Fixed, } \\
95 \% \mathrm{CI} \text { ) }\end{array}$ & $14.40[11.91,16.90]$ \\
\hline
\end{tabular}




\begin{tabular}{|c|c|c|c|c|}
\hline Outcome or subgroup title & No. of studies & No. of participants & Statistical method & Effect size \\
\hline $\begin{array}{l}\text { 7.1 Partially reversible } \\
\text { (mixed population) }\end{array}$ & 0 & & $\begin{array}{l}\% \text { increase (Fixed, } \\
95 \% \mathrm{CI} \text { ) }\end{array}$ & Not estimable \\
\hline 7.2 Poorly reversible & 2 & & $\begin{array}{l}\% \text { increase (Fixed, } \\
95 \% \mathrm{CI} \text { ) }\end{array}$ & $14.40[11.91,16.90]$ \\
\hline $\begin{array}{l}8 \text { Adverse events - 'serious } \\
\text { events' }\end{array}$ & 2 & 923 & $\begin{array}{l}\text { Odds Ratio (M-H, } \\
\text { Fixed, } 95 \% \mathrm{CI})\end{array}$ & $1.06[0.78,1.45]$ \\
\hline $\begin{array}{l}8.1 \text { Partially reversible } \\
\text { population (mixed } \\
\text { population) }\end{array}$ & 0 & 0 & $\begin{array}{l}\text { Odds Ratio (M-H, } \\
\text { Fixed, } 95 \% \mathrm{CI})\end{array}$ & Not estimable \\
\hline $\begin{array}{l}8.2 \text { Poorly reversible } \\
\text { population }\end{array}$ & 2 & 923 & $\begin{array}{l}\text { Odds Ratio (M-H, } \\
\text { Fixed, } 95 \% \text { CI) }\end{array}$ & $1.06[0.78,1.45]$ \\
\hline $\begin{array}{l}9 \text { Adverse events - } \\
\text { candidiasis }\end{array}$ & 0 & & $\begin{array}{l}\text { Odds Ratio (M-H, } \\
\text { Fixed, } 95 \% \mathrm{CI})\end{array}$ & Totals not selected \\
\hline $\begin{array}{l}9.1 \text { Partially reversible } \\
\text { population (mixed } \\
\text { population) }\end{array}$ & 0 & & $\begin{array}{l}\text { Odds Ratio (M-H, } \\
\text { Fixed, } 95 \% \mathrm{CI})\end{array}$ & Not estimable \\
\hline $\begin{array}{l}9.2 \text { Poorly reversible } \\
\text { population }\end{array}$ & 0 & & $\begin{array}{l}\text { Odds Ratio (M-H, } \\
\text { Fixed, } 95 \% \text { CI) }\end{array}$ & Not estimable \\
\hline $\begin{array}{l}10 \text { Adverse events - } \\
\text { pneumonia }\end{array}$ & 1 & 510 & $\begin{array}{l}\text { Odds Ratio (M-H, } \\
\text { Fixed, } 95 \% \mathrm{CI})\end{array}$ & $4.13[0.87,19.64]$ \\
\hline $\begin{array}{l}11 \text { Withdrawals due to } \\
\text { worsening COPD symptoms }\end{array}$ & 2 & 923 & $\begin{array}{l}\text { Odds Ratio (M-H, } \\
\text { Fixed, } 95 \% \text { CI) }\end{array}$ & $0.40[0.28,0.58]$ \\
\hline $\begin{array}{l}11.1 \text { Partially reversible } \\
\text { population (mixed } \\
\text { population) }\end{array}$ & 0 & 0 & $\begin{array}{l}\text { Odds Ratio (M-H, } \\
\text { Fixed, } 95 \% \text { CI) }\end{array}$ & Not estimable \\
\hline $\begin{array}{l}11.2 \text { Poorly reversible } \\
\text { population }\end{array}$ & 2 & 923 & $\begin{array}{l}\text { Odds Ratio (M-H, } \\
\text { Fixed, } 95 \% \mathrm{CI})\end{array}$ & $0.40[0.28,0.58]$ \\
\hline $\begin{array}{l}12 \text { Withdrawals due to } \\
\text { adverse events }\end{array}$ & 2 & 923 & $\begin{array}{l}\text { Odds Ratio (M-H, } \\
\text { Fixed, } 95 \% \mathrm{CI})\end{array}$ & $1.30[0.78,2.18]$ \\
\hline $\begin{array}{l}12.1 \text { Partially reversible } \\
\text { population (mixed } \\
\text { population) }\end{array}$ & 0 & 0 & $\begin{array}{l}\text { Odds Ratio (M-H, } \\
\text { Fixed, } 95 \% \mathrm{CI})\end{array}$ & Not estimable \\
\hline $\begin{array}{l}12.2 \text { Poorly reversible } \\
\text { population }\end{array}$ & 2 & 923 & $\begin{array}{l}\text { Odds Ratio (M-H, } \\
\text { Fixed, } 95 \% \mathrm{CI})\end{array}$ & $1.30[0.78,2.18]$ \\
\hline 13 Mortality & 2 & 923 & $\begin{array}{l}\text { Odds Ratio (M-H, } \\
\text { Fixed, } 95 \% \mathrm{CI})\end{array}$ & $0.78[0.35,1.73]$ \\
\hline $\begin{array}{l}13.1 \text { Mortality as primary } \\
\text { outcome }\end{array}$ & 0 & 0 & $\begin{array}{l}\text { Odds Ratio (M-H, } \\
\text { Fixed, } 95 \% \mathrm{CI})\end{array}$ & Not estimable \\
\hline $\begin{array}{l}\text { 13.2 Mortality data } \\
\text { collected as secondary/ } \\
\text { unpublished outcome }\end{array}$ & 2 & 923 & $\begin{array}{l}\text { Odds Ratio (M-H, } \\
\text { Fixed, } 95 \% \mathrm{CI})\end{array}$ & $0.78[0.35,1.73]$ \\
\hline
\end{tabular}




\section{Analysis 1.1}

\section{Comparison 1 Combined inhalers versus Placebo} (Primary Outcomes), Outcome 1 Exacerbation Rates with combined inhalers $v$. placebo

Review: Combined corticosteroid and long-acting beta-agonist in one inhaler versus placebo for chronic obstructive pulm onary disease Comparison: 1 Combined inhalers versus Placebo (Primaiy Outcomes)

Outcome: 1 Exacebation Rates with combined inhalers v. placebo

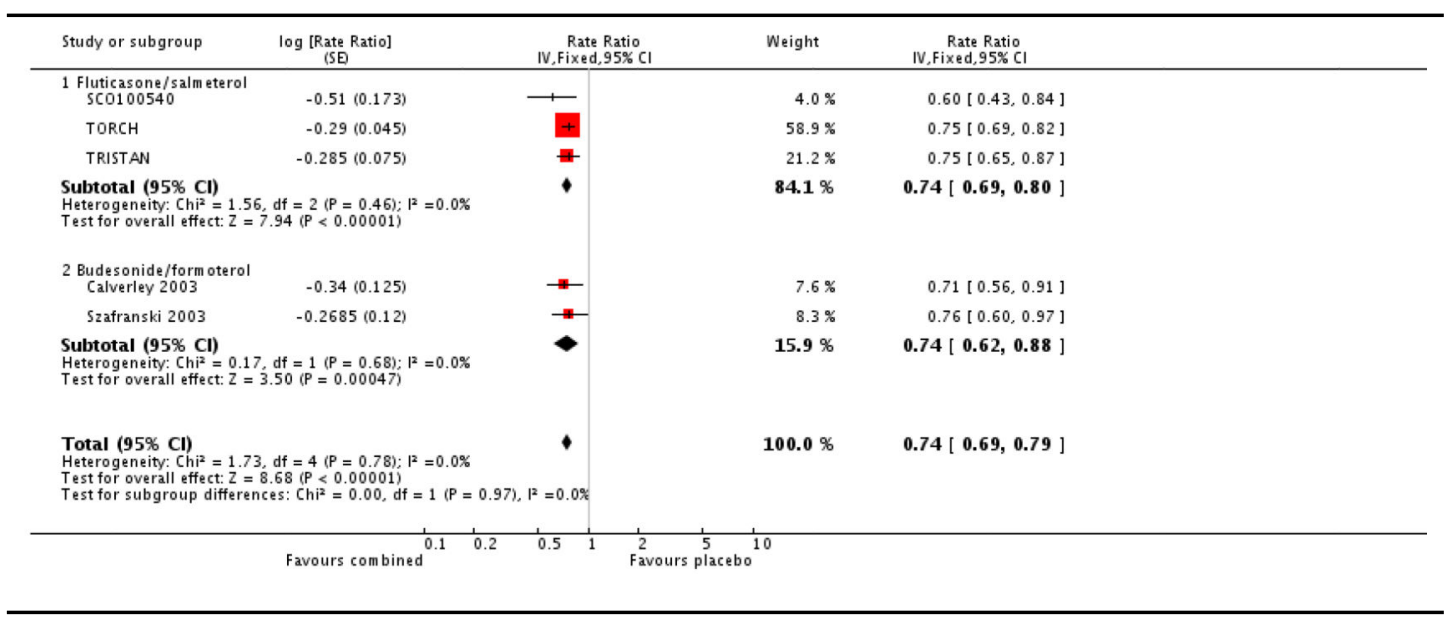




\section{Analysis 1.2}

\section{Comparison 1 Combined inhalers versus Placebo (Primary Outcomes), Outcome 2 Mortality}

Review: Combined corticosteroid and long-acting beta-agonist in one inhaler versus placebo for chronic obstructive pulmonary disease Comparison: 1 Combined inhalers versus Placebo (Primaiy Outcomes)

Outcome: 2 Mortality

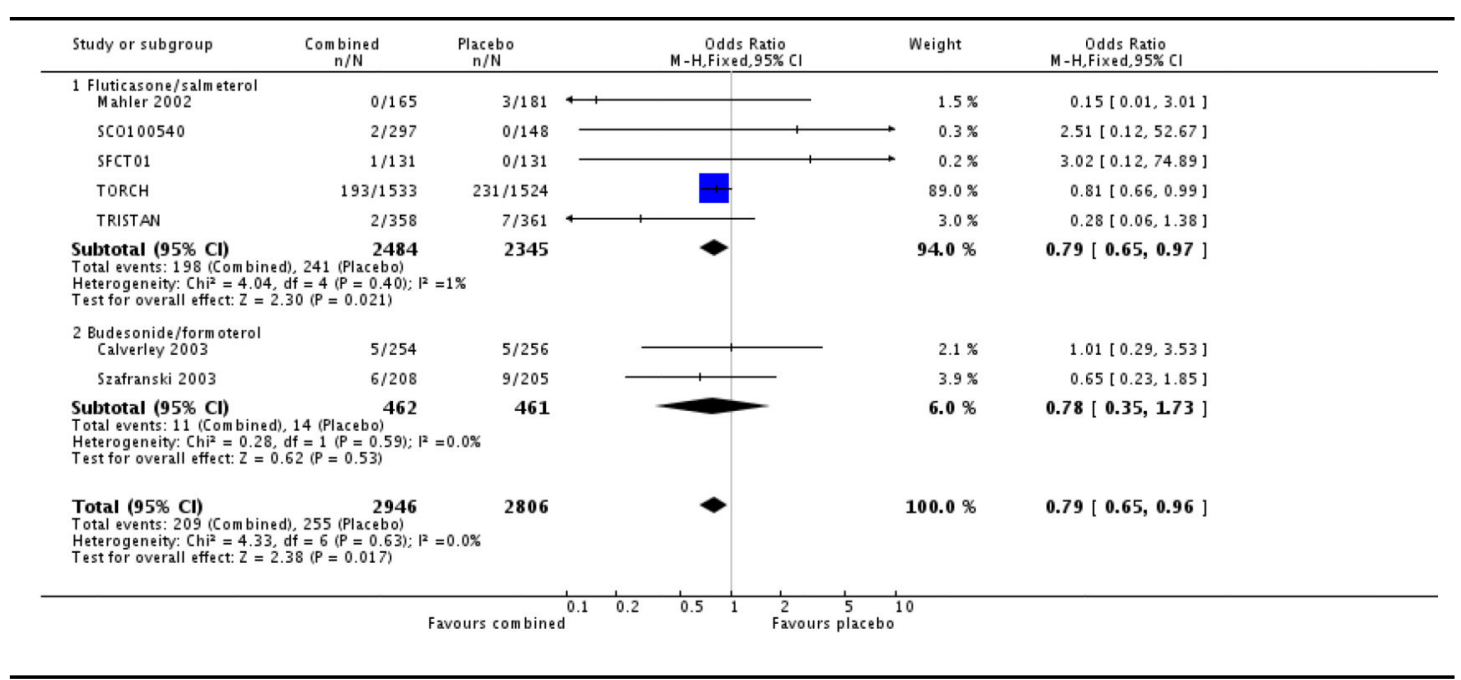


Analysis 1.3

\section{Comparison 1 Combined inhalers versus Placebo (Primary Outcomes), Outcome 3 Pneumonia}

Review: Combined corticosteroid and long-acting beta-agonist in one inhaler versus placebo for chronic obstructive pulmonary disease Comparison: 1 Combined inhalers versus Placebo (Primary Outcomes)

Outcome: 3 Pneumonia

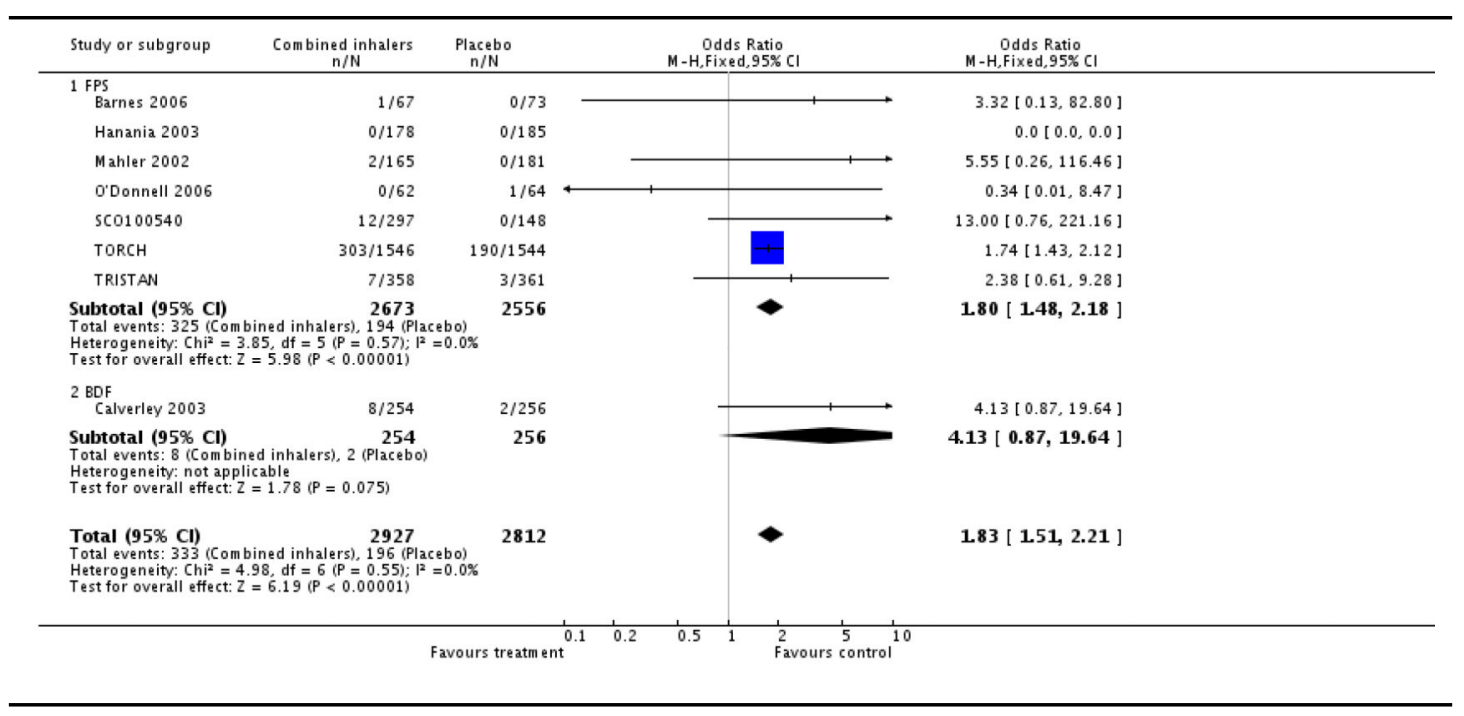




\section{Analysis 2.1}

\section{Comparison 2 Fluticasone/salmeterol (FPS) versus placebo (PLA), Outcome 1 Number of participants with one or more exacerbation}

Review: Combined corticosteroid and long-acting beta-agonist in one inhaler versus placebo for chronic obstructive pulmonary disease Comparison: 2 Fluticasone/salmeterol (FPS) versus placebo (PLS)

Outcome: 1 Number of participants with one or more exacerbation

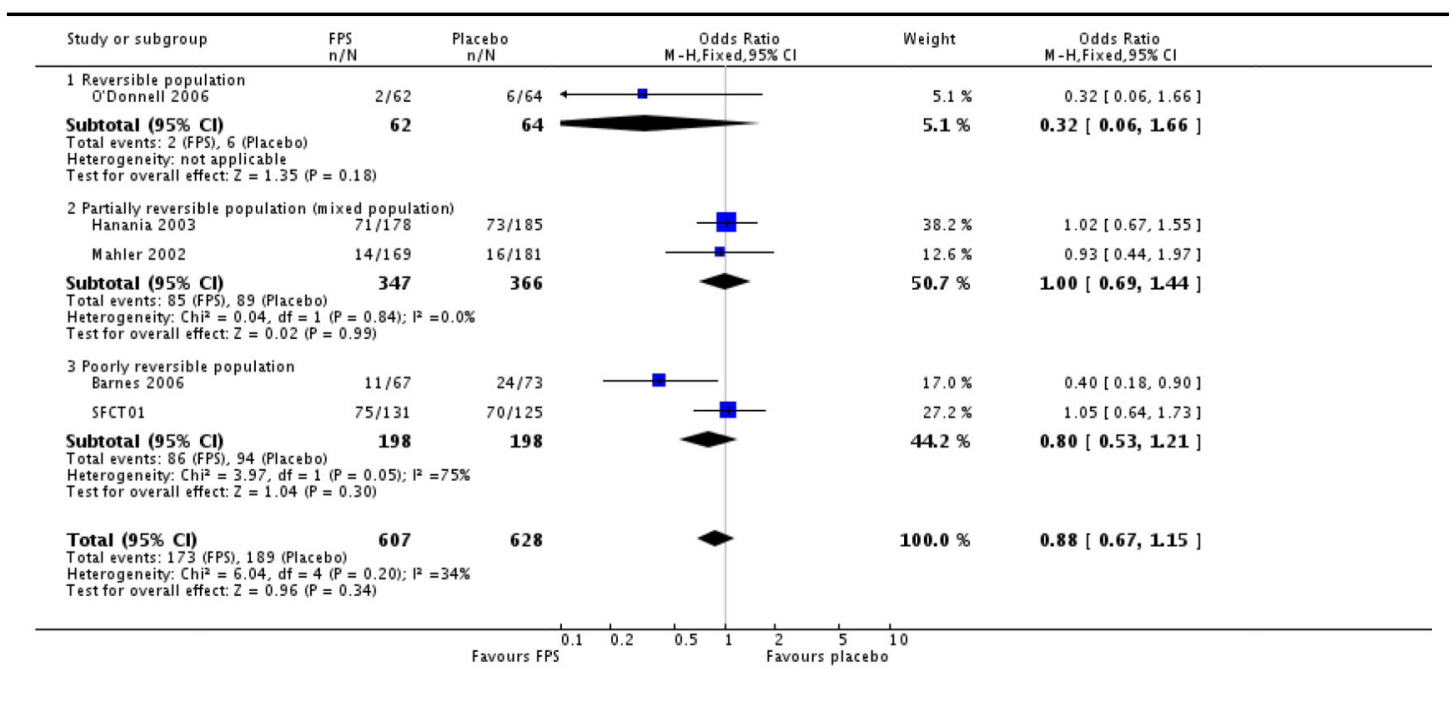

Analysis 2.2

Comparison 2 Fluticasone/salmeterol (FPS) versus placebo (PLA), Outcome 2 End of treatment mean number of exacerbations per participant

Review: Combined corticosteroid and long-acting beta-agonist in one inhaler versus placebo for chronic obstructive pulmonary disease Comparison: 2 Fluticasone/salmeterol (FPS) versus placebo (PLS)

Outcome: 2 End of treatment mean number of exacerbations per paiticipant

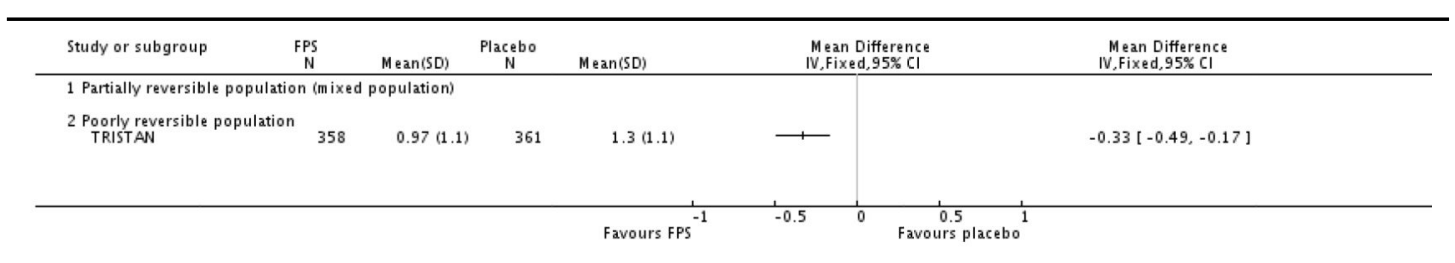


Analysis 2.3

\section{Comparison 2 Fluticasone/salmeterol (FPS) versus placebo (PLA), Outcome 3 Exacerbations}

Review: Combined corticosteroid and long-acting beta-agonist in one inhaler versus placebo for chronic obstructive pulmonary disease Comparison: 2 Fluticasone/salmeterol (FPS) versus placebo (PLS)

Outcome: 3 Exacerbations

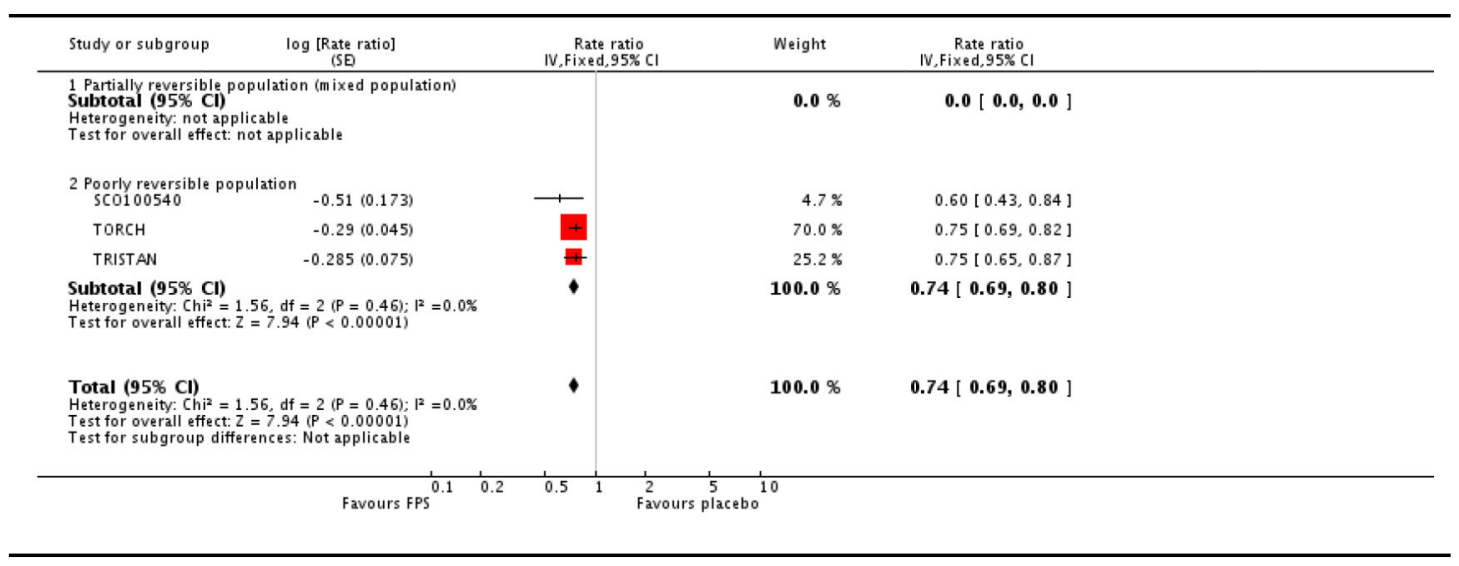




\section{Analysis 2.4}

\section{Comparison 2 Fluticasone/salmeterol (FPS) versus placebo (PLA), Outcome 4 Number of exacerbations type}

Review: Combined corticosteroid and long-acting beta-agonist in one inhaler versus placebo for chronic obstructive pulmonary disease Comparison: 2 Fluticasone/salmeterol (FPS) versus placebo (PLA)

Outcome: 4 Number of exacerbations by type

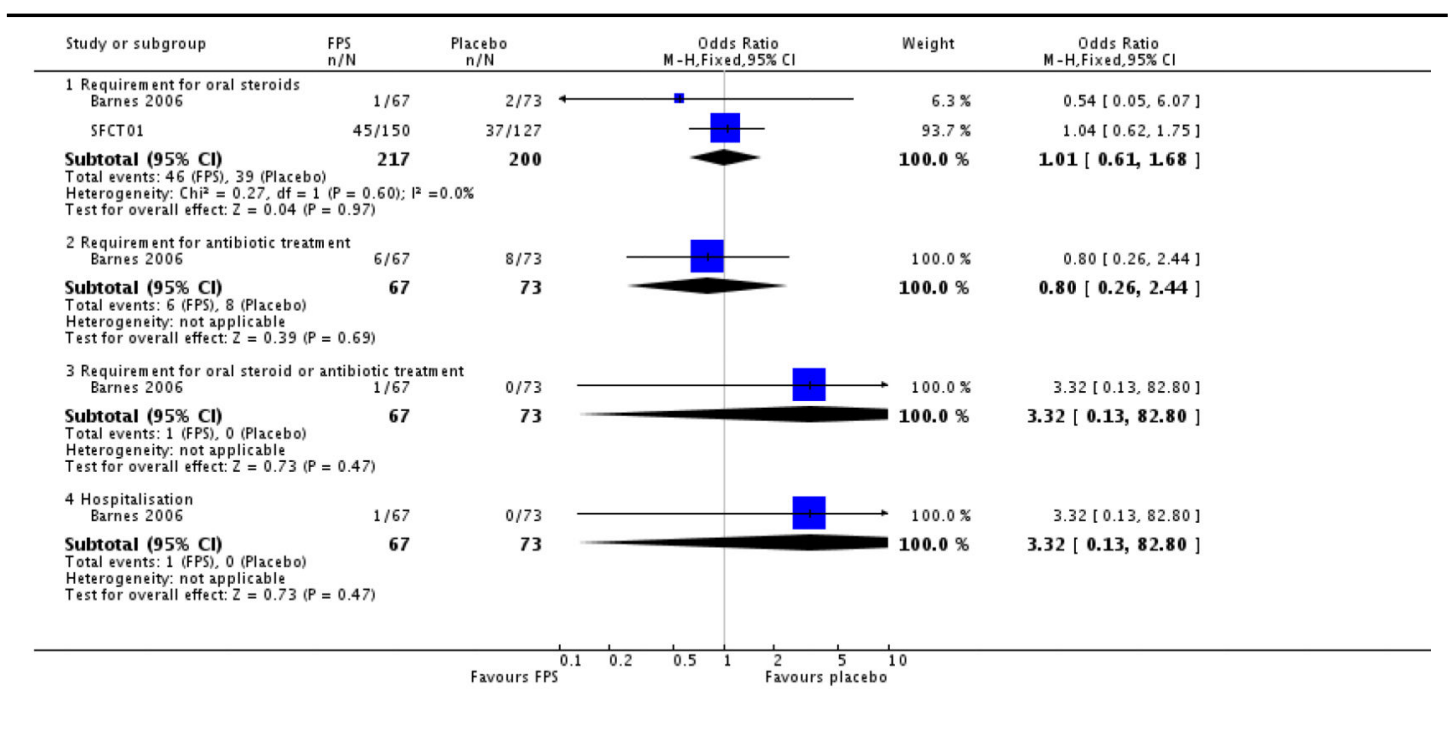


Analysis 2.5

\section{Comparison 2 Fluticasone/salmeterol (FPS) versus placebo (PLA), Outcome 5 Exacerbations type}

Review: Combined corticosteroid and long-acting beta-agonist in one inhaler versus placebo for chronic obstructive pulmonary disease Comparison: 2 Fluticasone/salmeterol (FPS) versus placebo (PLA)

Outcome: 5 Exacerbations by type

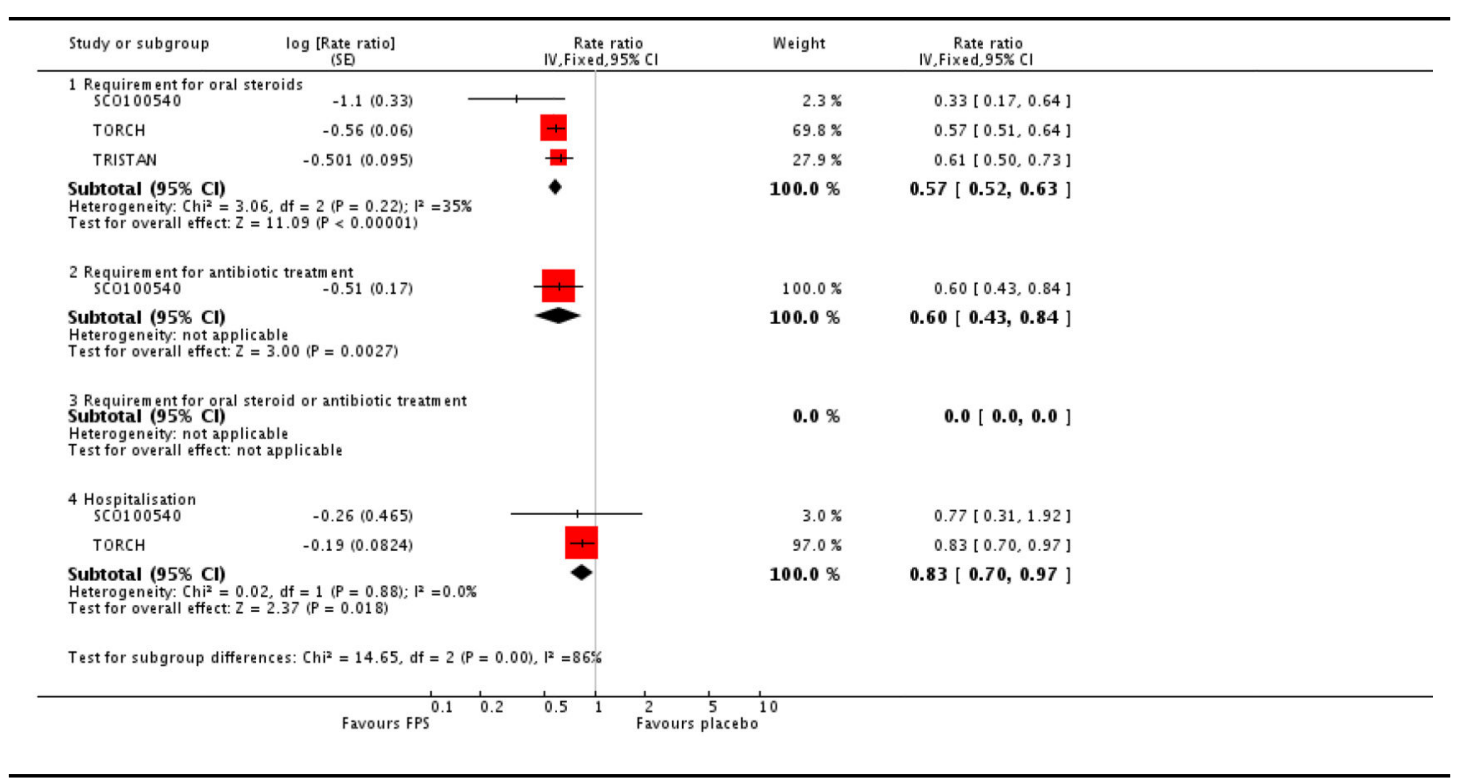


Analysis 2.6

\section{Comparison 2 Fluticasone/salmeterol (FPS) versus placebo (PLA), Outcome 6 Mortality}

Review: Combined corticosteroid and long-acting beta-agonist in one inhaler versus placebo for chronic obstructive pulmonary disease Comparison: 2 Fluticasone/salmeterol (FPS) versus placebo (PLA)

Outcome: 6 Mortality

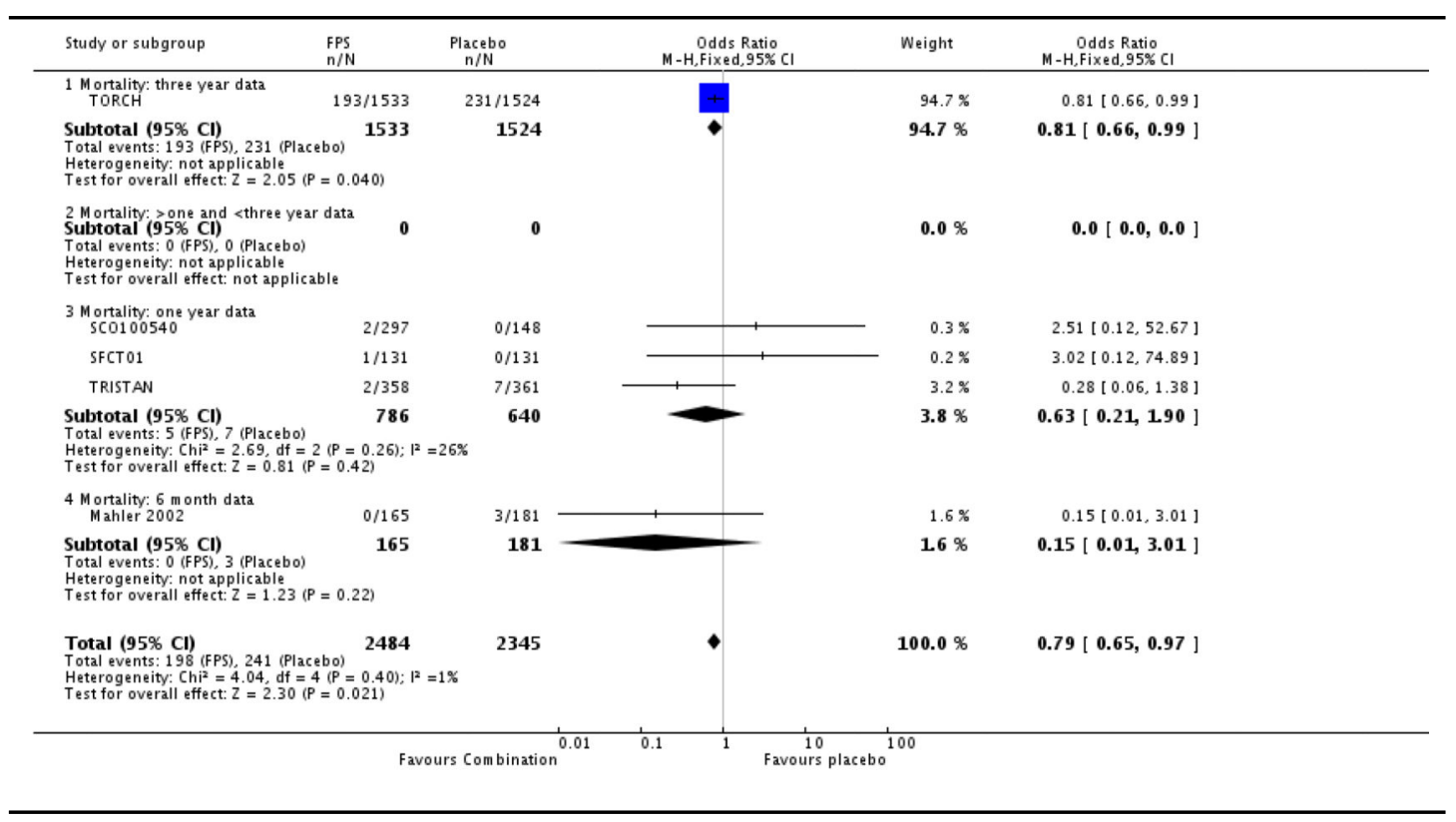




\section{Analysis 2.7}

\section{Comparison 2 Fluticasone/salmeterol (FPS) versus} placebo (PLA), Outcome 7 Change from baseline in St George's Respiratory Questionnaire (total score)

Review: Combined corticosteroid and long-acting beta-agonist in one inhaler versus placebo for chronic obstructive pulmonary disease Comparison: 2 Fluticasone/salmeterol (FPS) versus placebo (PLS)

Outcome: 7 Change from baseline in St George's Respiratory Questionnaire (total score)

\begin{tabular}{|c|c|c|c|}
\hline Study or subgroup & $\begin{array}{r}\text { SCRQ units } \\
\text { IV, Fixed, } 95 \% \mathrm{Cl} \\
\end{array}$ & Weight & $\begin{array}{r}\text { SCRQ units } \\
\text { IV.Fixed, } 95 \% \mathrm{CI}\end{array}$ \\
\hline $\begin{array}{l}1 \text { Partially reversible population (mixed population) } \\
\text { Subtotal ( } 95 \% \text { CI) } \\
\text { Heterogeneneity; not applicable } \\
\text { Test for overall effect: not applicable }\end{array}$ & & $0.0 \%$ & $0.0[0.0,0.0]$ \\
\hline \multicolumn{4}{|l|}{2 Poorly reversible population } \\
\hline$-2.92(1.9719)$ & & $3.4 \%$ & $-2.92[-6.78,0.94]$ \\
\hline$-3.1(0.51)$ & 7 & $51.1 \%$ & $-3.10[-4.10,-2.10]$ \\
\hline$-2.2(0.58)$ & \# & $39.5 \%$ & $-2.20[-3.34,-1.06]$ \\
\hline $\begin{array}{l}\text { Subtotal }\left(95 \% \mathrm{Cl}^{2}\right) \\
\text { Heterogeneity: Chi }=5.22, \mathrm{df}=3(\mathrm{P}=0.16) ;\left.\right|^{2}=43 \% \\
\text { Test for overall effect: } z=7.94(\mathrm{P}<0.00001)\end{array}$ & $\bullet$ & $100.0 \%$ & $-2.90[-3.61,-2.18]$ \\
\hline $\begin{array}{l}\text { Total }(95 \% \mathrm{Cl}) \\
\text { Heterogeneity: Chi }=5.22, \mathrm{df}=3(\mathrm{P}=0.16) ; 1^{2}=43 \% \\
\text { Test for overall effectit } Z=7.94(\mathrm{P}<0.00001) \\
\text { Test for subgroup differences: Not applicable }\end{array}$ & $\bullet$ & $100.0 \%$ & $-2.90[-3.61,-2.18]$ \\
\hline${\text { Favours } \mathrm{FPS}^{-10}}^{-10}$ & $\frac{1}{0}$ & 10 & \\
\hline
\end{tabular}

\section{Analysis 2.8}

Comparison 2 Fluticasone/salmeterol (FPS) versus placebo (PLA), Outcome 8 Change from baseline in St George's Respiratory Questionnaire (domain symptoms)

Review: Combined corticosteroid and long-acting beta-agonist in one inhaler versus placebo for chronic obstructive pulmonary disease Comparison: 2 Fluticasone/salmeterol (FPS) versus placebo (PLA) Outcome: 8 Change from baseline in St George's Respiratory Questionnaire (domain symptoms)

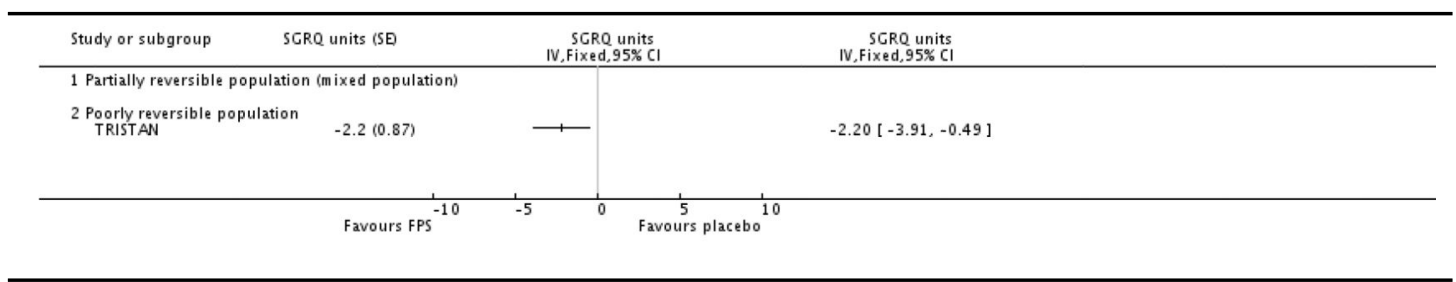




\section{Analysis 2.9}

Comparison 2 Fluticasone/salmeterol (FPS) versus placebo (PLA), Outcome 9 Change from baseline in St George's Respiratory Questionnaire (domain symptoms)

Review: Combined corticosteroid and long-acting beta-agonist in one inhaler versus placebo for chronic obstructive pulmonary disease Comparison: 2 Fluticasone/salmeterol (FPS) versus placebo (PLA) Outcome: 9 Change from baseline in St George's Respiratory Questionnaire (domain symptoms)

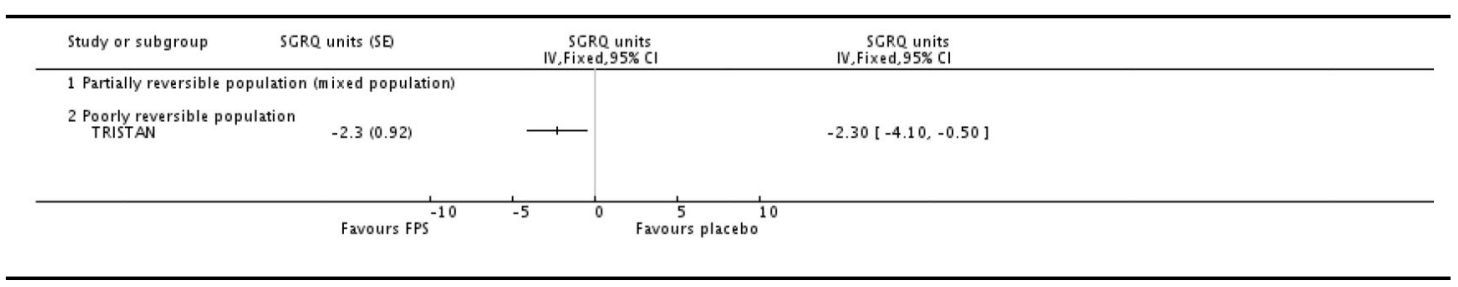

Analysis 2.10

Comparison 2 Fluticasone/salmeterol (FPS) versus placebo (PLA), Outcome 10 Change from baseline in $\mathbf{S t}$ George's Respiratory Questionnaire (domain - impact)

Review: Combined corticosteroid and long-acting beta-agonist in one inhaler versus placebo for chronic obstructive pulmonary disease Comparison: 2 Fluticasone/salmeterol (FPS) versus placebo (PLA) Outcome: 10 Change from baseline in St George's Respiratory Questionnaire (domain impact)

\begin{tabular}{|c|c|c|c|c|}
\hline Study or subgroup & $Q$ units (SE) & \multicolumn{2}{|c|}{$\begin{array}{r}\text { SCRQ units } \\
\text { IV,Fixed, } 95 \% \mathrm{CI} \\
\end{array}$} & $\begin{array}{r}\text { SCRQ units } \\
\text { IV, Fixed, } 95 \% \mathrm{CI} \\
\end{array}$ \\
\hline \multicolumn{2}{|c|}{1 Partially reversible population (mixed population) } & & & \\
\hline \multirow[t]{2}{*}{$\begin{array}{l}2 \text { Poorly reversible population } \\
\text { TRISTAN }\end{array}$} & $-2.7(0.714)$ & $\longrightarrow$ & & $-2.70[-4.10,-1.30]$ \\
\hline & Favours FPS $^{-10}$ & -5 & Favo & \\
\hline
\end{tabular}




\section{Analysis 2.11}

Comparison 2 Fluticasone/salmeterol (FPS) versus

placebo (PLA), Outcome 11 End of treatment St

George's Respiratory Questionnaire scores (total score)

Review: Combined corticosteroid and long-acting beta-agonist in one inhaler versus placebo for chronic obstructive pulmonary disease

Comparison: 2 Fluticasone/salmeterol (FPS) versus placebo (PLA)

Outcome: 11 End of treatment St George's Respiratory Questionnaire scores (total score)

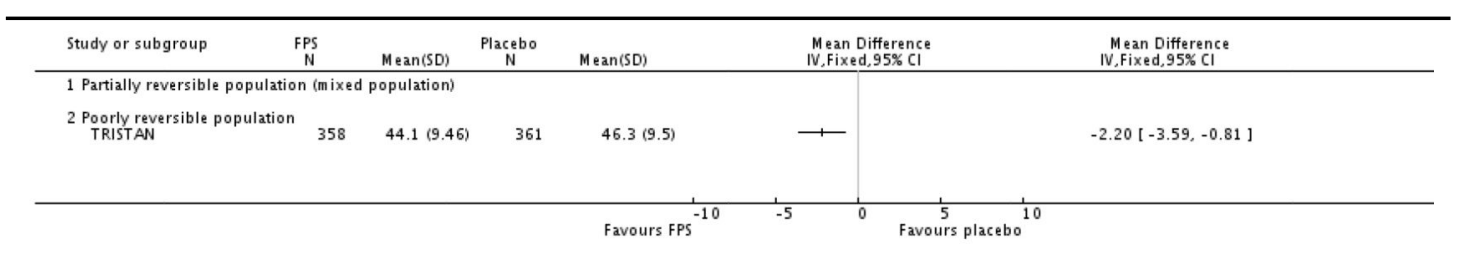

Analysis 2.12

Comparison 2 Fluticasone/salmeterol (FPS) versus

placebo (PLA), Outcome 12 End of treatment St

George's Respiratory Questionnaire scores (domain breathlessness)

Review: Combined corticosteroid and long-acting beta-agonist in one inhaler versus placebo for chronic obstructive pulmonary disease Comparison: 2 Fluticasone/salmeterol (FPS) versus placebo (PLA)

Outcome: 12 End of treatm ent St George's Respiratory Questionnaire scores (domain breathlessness)

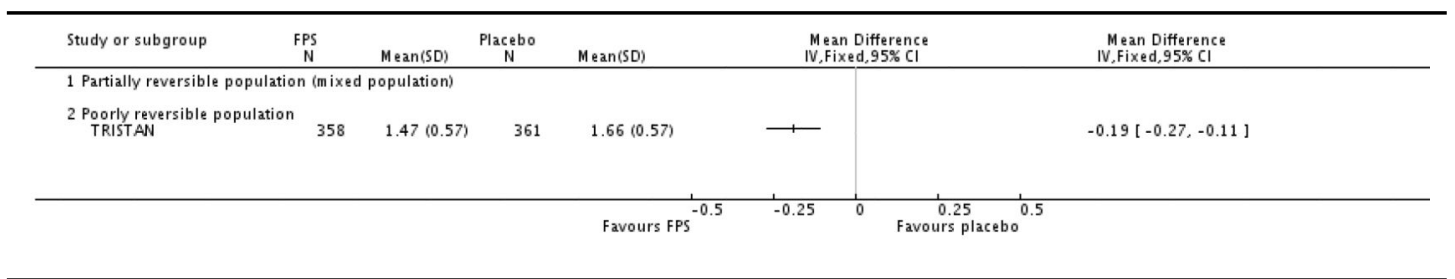


Analysis 2.13

Comparison 2 Fluticasone/salmeterol (FPS) versus placebo (PLA), Outcome 13 Change from baseline in Canadian Respiratory Disease Questionnaire scores

Review: Combined corticosteroid and long-acting beta-agonist in one inhaler versus placebo for chronic obstructive pulmonary disease Comparison: 2 Fluticasone/salmeterol (FPS) versus placebo (PLA)

Outcome: 13 Change from baseline in Canadian Respiratoiy Disease Questionnaire scores

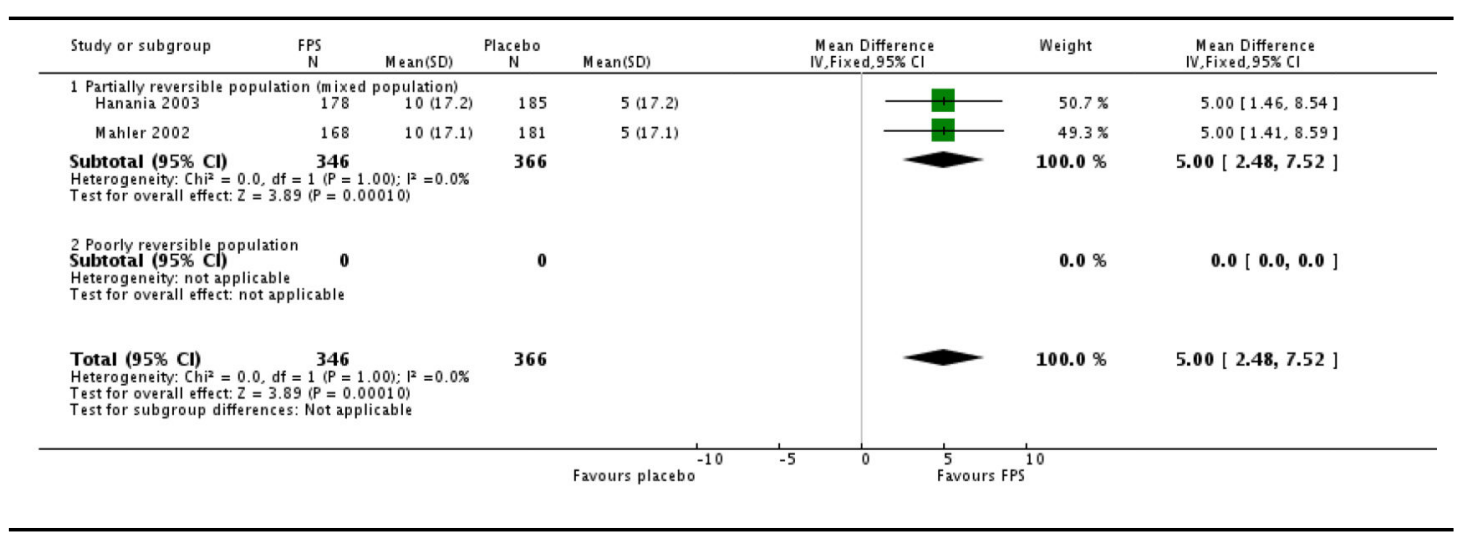

Analysis 2.14

Comparison 2 Fluticasone/salmeterol (FPS) versus placebo (PLA), Outcome 14 Change from baseline in Transitional Dyspnoea Index (TDI)

Review: Combined corticosteroid and long-acting beta-agonist in one inhaler versus placebo for chronic obstructive pulmonary disease Comparison: 2 Fluticasone/salmeterol (FPS) versus placebo (PLA) Outcome: 14 Change from baseline in Transitional Dyspnoea Index (TDI) scores

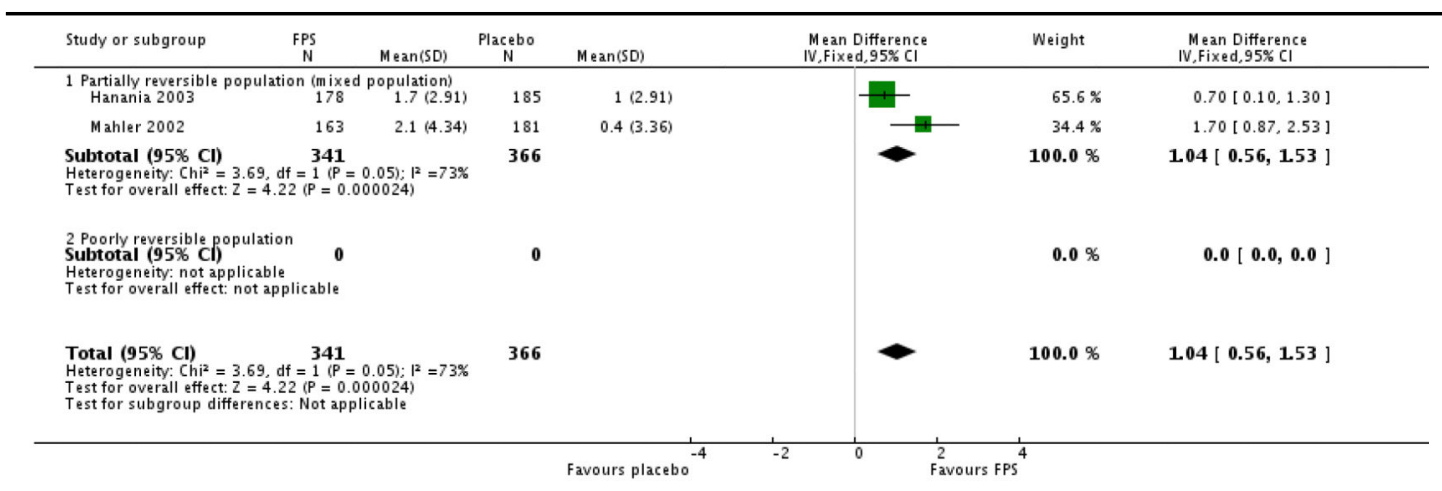




\section{Analysis 2.15}

Comparison 2 Fluticasone/salmeterol (FPS) versus placebo (PLA), Outcome 15 End of treatment symptom scores

Review: Combined corticosteroid and long-acting beta-agonist in one inhaler versus placebo for chronic obstructive pulmonary disease Comparison: 2 Fluticasone/salmeterol (FPS) versus placebo (PLA)

Outcome: 15 End of treatment symptom scores

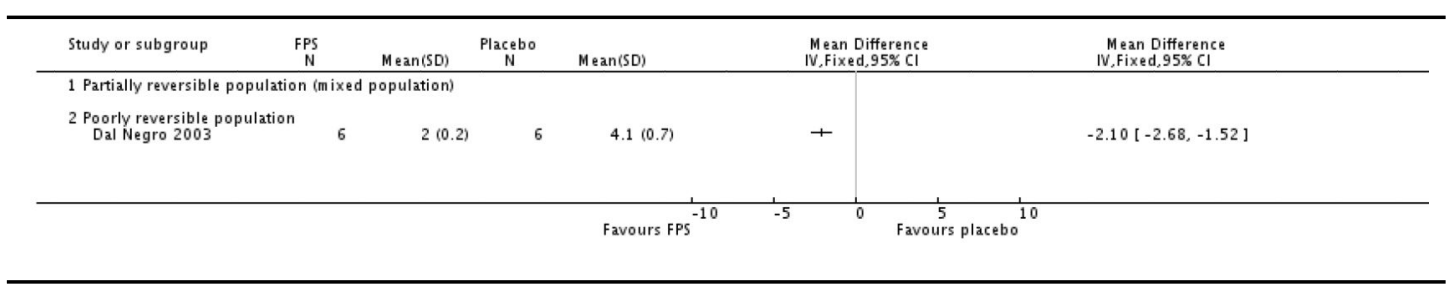

\section{Analysis 2.16}

Comparison 2 Fluticasone/salmeterol (FPS) versus placebo (PLA), Outcome 16 Change from baseline in predose FEV1

Review: Combined corticosteroid and long-acting beta-agonist in one inhaler versus placebo for chronic obstructive pulmonary disease Comparison: 2 Fluticasone/salmeterol (FPS) versus placebo (PLA) Outcome: 16 Change from baseline in predose FEV1

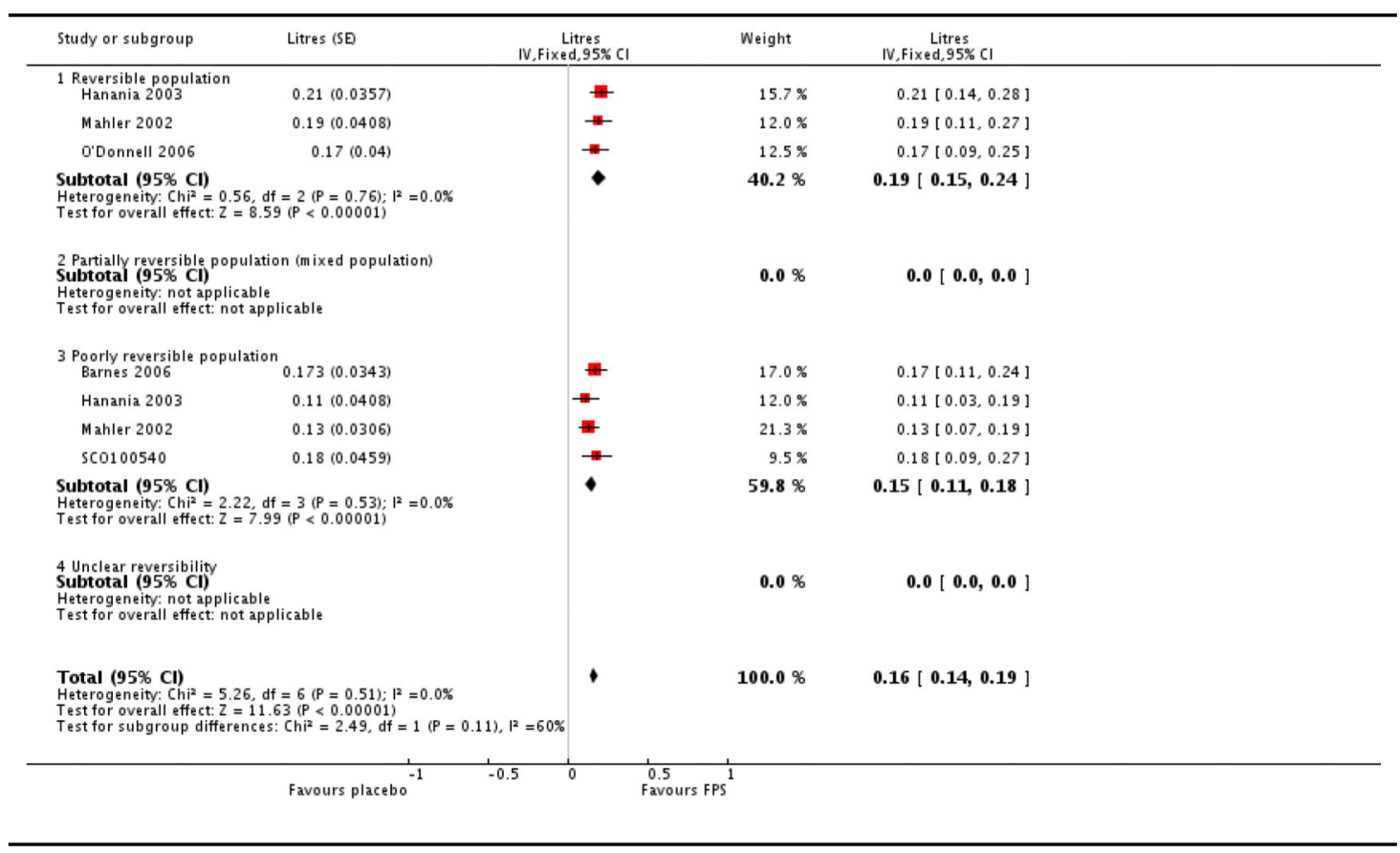


Analysis 2.17

Comparison 2 Fluticasone/salmeterol (FPS) versus placebo (PLA), Outcome 17 Change from baseline in postdose FEV1

Review: Combined corticosteroid and long-acting beta-agonist in one inhaler versus placebo for chronic obstructive pulmonary disease Comparison: 2 Fluticasone/salm eterol (FPS) versus placebo (PLA)

Outcome: 17 Change from baseline in postdose FEV1

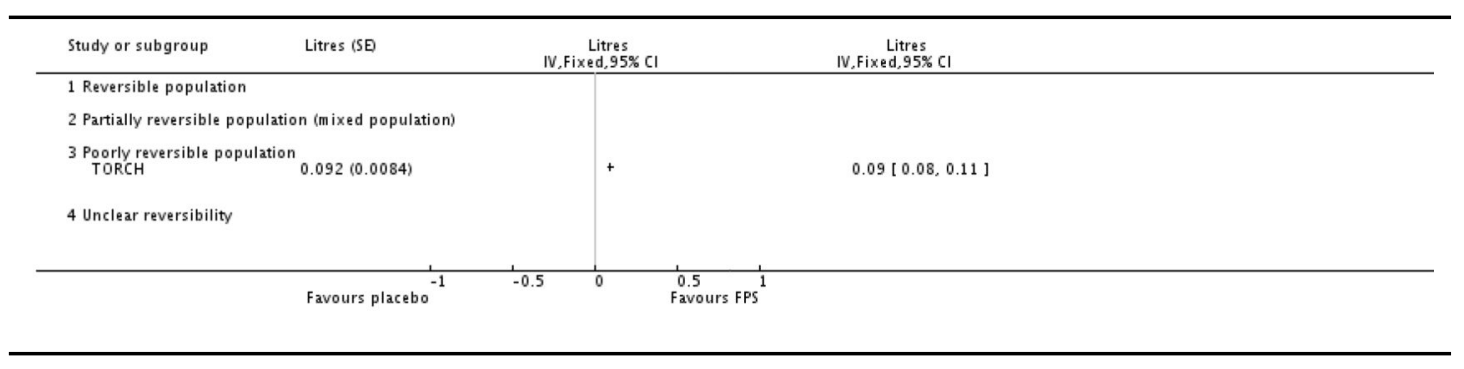

Analysis 2.18

Comparison 2 Fluticasone/salmeterol (FPS) versus placebo (PLA), Outcome 18 End of treatment FEV1 (\% predicted)

Review: Combined corticosteroid and long-acting beta-agonist in one inhaler versus placebo for chronic obstructive pulmonary disease Comparison: 2 Fluticasone/salmeterol (FPS) versus placebo (PLA) Outcome: 18 End of treatment FEV1 (\% predicted)

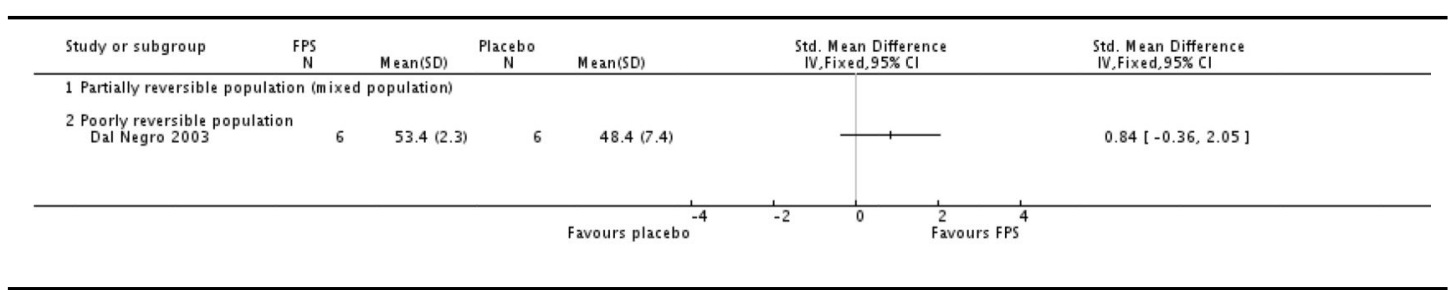




\section{Analysis 2.19}

Comparison 2 Fluticasone/salmeterol (FPS) versus placebo (PLA), Outcome 19 End of treatment FEV1

(Litres)

Review: Combined corticosteroid and long-acting beta-agonist in one inhaler versus placebo for chronic obstructive pulmonary disease Comparison: 2 Fluticasone/salmeterol (FPS) versus placebo (PLA)

Outcome: 19 End of treatment FEV1 (Litres)

\begin{tabular}{|c|c|c|c|}
\hline Study or subgroup & $\begin{array}{c}\text { Litres } \\
\text { IV, Fixed, } 95 \% \mathrm{Cl}\end{array}$ & Weight & $\begin{array}{c}\text { Litres } \\
\text { IV, Fixed, } 95 \% \mathrm{Cl}\end{array}$ \\
\hline $\begin{array}{l}1 \text { Partially reversible population (mixed population) } \\
\text { Subtotal ( } 95 \% \text { CI) } \\
\text { Heterogeneity not applicable } \\
\text { Test for overall effect: not applicable }\end{array}$ & & $0.0 \%$ & $0.0[0.0,0.0]$ \\
\hline $\begin{array}{l}2 \text { Poorly reversible population } \\
\text { SFCT 01 }\end{array} 0.103(0.044)$ & 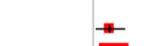 & $11.2 \%$ & $0.10[0.02,0.19]$ \\
\hline $0.132(0.0156)$ & + & $88.8 \%$ & $0.13[0.10,0.16]$ \\
\hline $\begin{array}{l}\text { Total }(95 \% \mathrm{Cl}) \\
\text { Heterogeneity: } \mathrm{Chi}^{2}=0.39, \mathrm{df}=1(\mathrm{P}=0.53) ;\left.\right|^{2}=0.0 \% \\
\text { Test for overail effectif } Z=8.76 \quad(\mathrm{P}<0.00001) \\
\text { Test for sub group differences: Not applicable }\end{array}$ & - & $100.0 \%$ & $0.13[0.10,0.16]$ \\
\hline Favours placebo $^{-1}$ & -0.5 & $\begin{array}{l}1 \\
0.5 \\
\text { Favours FPS }\end{array}$ & \\
\hline
\end{tabular}

Analysis 2.20

Comparison 2 Fluticasone/salmeterol (FPS) versus placebo (PLA), Outcome 20 End of treatment am PEF (L/min)

Review: Combined corticosteroid and long-acting beta-agonist in one inhaler versus placebo for chronic obstructive pulmonary disease Comparison: 2 Fluticasone/salmeterol (FPS) versus placebo (PLA) Outcome: 20 End of treatment am PEF (L/min)

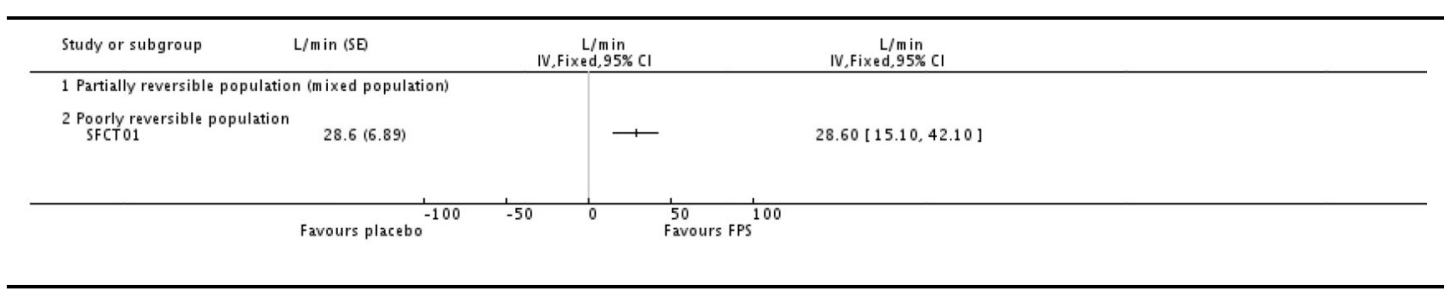




\section{Analysis 2.21}

Comparison 2 Fluticasone/salmeterol (FPS) versus placebo (PLA), Outcome 21 End of treatment shuttle walk test

Review: Combined corticosteroid and long-acting beta-agonist in one inhaler versus placebo for chronic obstructive pulmonary disease Comparison: 2 Fluticasone/salmeterol (FPS) versus placebo (PLA)

Outcome: 21 End of treatment shuttle walk test

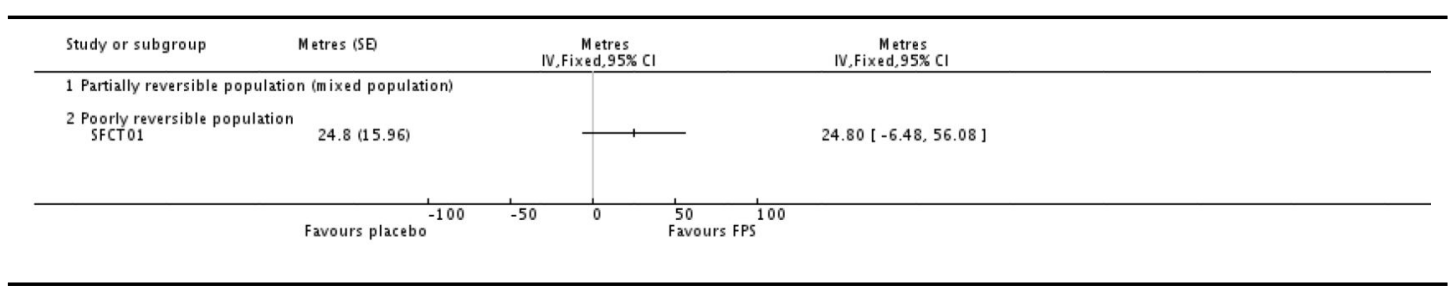

\section{Analysis 2.22}

Comparison 2 Fluticasone/salmeterol (FPS) versus placebo (PLA), Outcome 2 End of treatment rescue medication usage (puffs/day)

Review: Combined corticosteroid and long-acting beta-agonist in one inhaler versus placebo for chronic obstructive pulmonary disease Comparison: 2 Fluticasone/salmeterol (FPS) versus placebo (PLS)

Outcome: 22 End of treatment rescue medication usage (puffs/day)

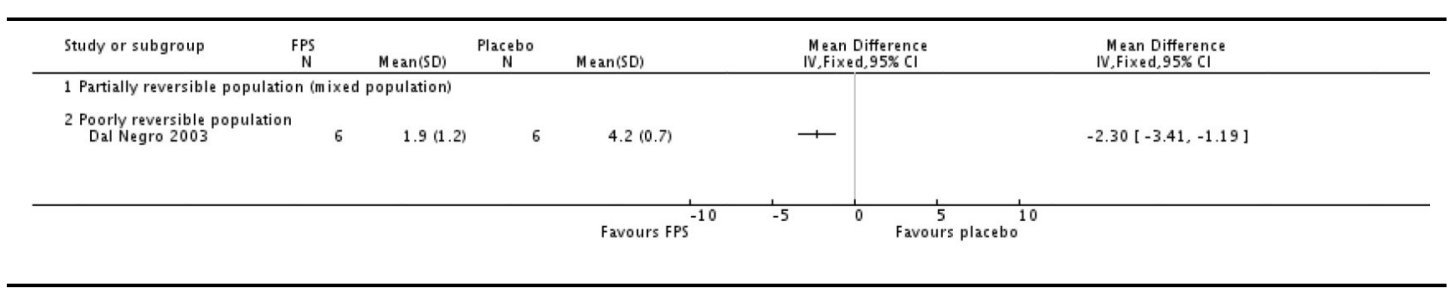


Analysis 2.23

Comparison 2 Fluticasone/salmeterol (FPS) versus placebo (PLA), Outcome 23 Change from baseline in rescue medication usage (puffs/day)

Review: Combined corticosteroid and long-acting beta-agonist in one inhaler versus placebo for chronic obstructive pulmonary disease Comparison: 2 Fluticasone/salmeterol (FPS) versus placebo (PLA)

Outcome: 23 Change from baseline in rescue medication usage (puffs/day)

\begin{tabular}{|c|c|c|c|c|c|c|}
\hline \multicolumn{2}{|c|}{$\begin{array}{l}\text { 1. Partially reversible population (mixed population) } \\
\text { Hanania } 2003 \\
178\end{array}$} & 185 & $0.1(3.35)$ & + & $85.0 \%$ & $-1.10[-1.79,-0.41]$ \\
\hline Mahler 2002 & $-1.2(7.7)$ & 179 & $0.5(7.7)$ & 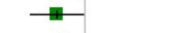 & $15.0 \%$ & $-1.70[-3.34,-0.06]$ \\
\hline \multicolumn{2}{|l|}{$\begin{array}{l}2 \text { Poorly reversible population } \\
\text { Subtotal }(95 \% \text { Cl) } \\
\text { Heterogeneity not applicable } \\
\text { Test for overall effect: not applicable }\end{array}$} & $\mathbf{0}$ & & & $0.0 \%$ & $0.0[0.0,0.0]$ \\
\hline \multirow{2}{*}{\multicolumn{2}{|c|}{$\begin{array}{l}\text { Total }(95 \% \mathrm{Cl}) \quad 339 \\
\text { Heterogeneity: Chi }=0.44, \mathrm{df}=1(\mathrm{P}=0.51) ; 1^{2}=0.0 \% \\
\text { Test for overall effectit } Z=3.67(P=0.00024) \\
\text { Test for subgroup differences: Not applicable }\end{array}$}} & 364 & & $\bullet$ & $100.0 \%$ & $-1.19[-1.83,-0.55]$ \\
\hline & & & Favours FPS $^{-10}$ & Favo & 10 & \\
\hline
\end{tabular}


Analysis 2.24

Comparison 2 Fluticasone/salmeterol (FPS) versus placebo (PLA), Outcome 24 Adverse events- any event

Review: Combined corticosteroid and long-acting beta-agonist in one inhaler versus placebo for chronic obstructive pulmonary disease Comparison: 2 Fluticasone/salmeterol (FPS) versus placebo (PLA)

Outcome: 24 Adverse events - any event

\begin{tabular}{|c|c|c|c|c|c|}
\hline $\begin{array}{l}1 \text { Reversible population } \\
\text { O'Donnell } 2006\end{array}$ & $26 / 62$ & $24 / 64$ & & $3.7 \%$ & $1.20[0.59,2.46]$ \\
\hline $\begin{array}{l}\text { Subtotal (95\% CI) } \\
\text { Total events } 26 \text { (FP), } 24 \text { (Placebo) } \\
\text { Heterogeneity: not applicable } \\
\text { Test for overall effect: } Z=0.51 \text { (P }\end{array}$ & $\begin{array}{l}62 \\
=0.61)\end{array}$ & 64 & & $3.7 \%$ & $1.20[0.59,2.46]$ \\
\hline \multicolumn{4}{|c|}{2 Partially reversible population (mixed population) } & $9.4 \%$ & $1.30[0.84,2.02]$ \\
\hline \multicolumn{6}{|c|}{$\begin{array}{l}\text { Total events: } 255 \text { (FPS), } 245 \text { (Placebo) } \\
\text { Heterogeneity: Chi }{ }^{2}=0.33, \mathrm{df}=1(\mathrm{P}=0.57) ; 1^{2}=0.0 \% \\
\text { Test for overall effect: } z=2.14(P=0.032)\end{array}$} \\
\hline $\begin{array}{l}3 \text { Poorly reversible population } \\
\text { Barnes } 2006\end{array}$ & $38 / 67$ & $41 / 73$ & & $4.5 \%$ & $1.02[0.52,2.00]$ \\
\hline SCO100540 & $158 / 297$ & $78 / 148$ & 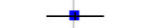 & $13.0 \%$ & $1.02[0.69,1.51]$ \\
\hline SFCT01 & $72 / 131$ & $60 / 125$ & $\longrightarrow$ & $7.4 \%$ & $1.32[0.81,2.16]$ \\
\hline \multicolumn{6}{|c|}{$\begin{array}{l}\text { Total events: } 2215 \text { (FPS), } 2116 \text { (Place bo) } \\
\text { Heterogeneity: Chi }=4.88 \text {, df }=7(\mathrm{P}=0.67): 1^{2}=0.0 \% \\
\text { Test for overall effect: } Z=1.35(P=0.18)\end{array}$} \\
\hline & & Favours FPs ${ }^{0.1}$ & $\frac{1}{2}$ & & \\
\hline
\end{tabular}


Analysis 2.25

Comparison 2 Fluticasone/salmeterol (FPS) versus placebo (PLA), Outcome 25 Adverse events candidiasis

Review: Combined corticosteroid and long-acting beta-agonist in one inhaler versus placebo for chronic obstructive pulmonary disease Comparison: 2 Fluticasone/salmeterol (FPS) versus placebo (PLA)

Outcome: 25 Adverse events - candidiasis

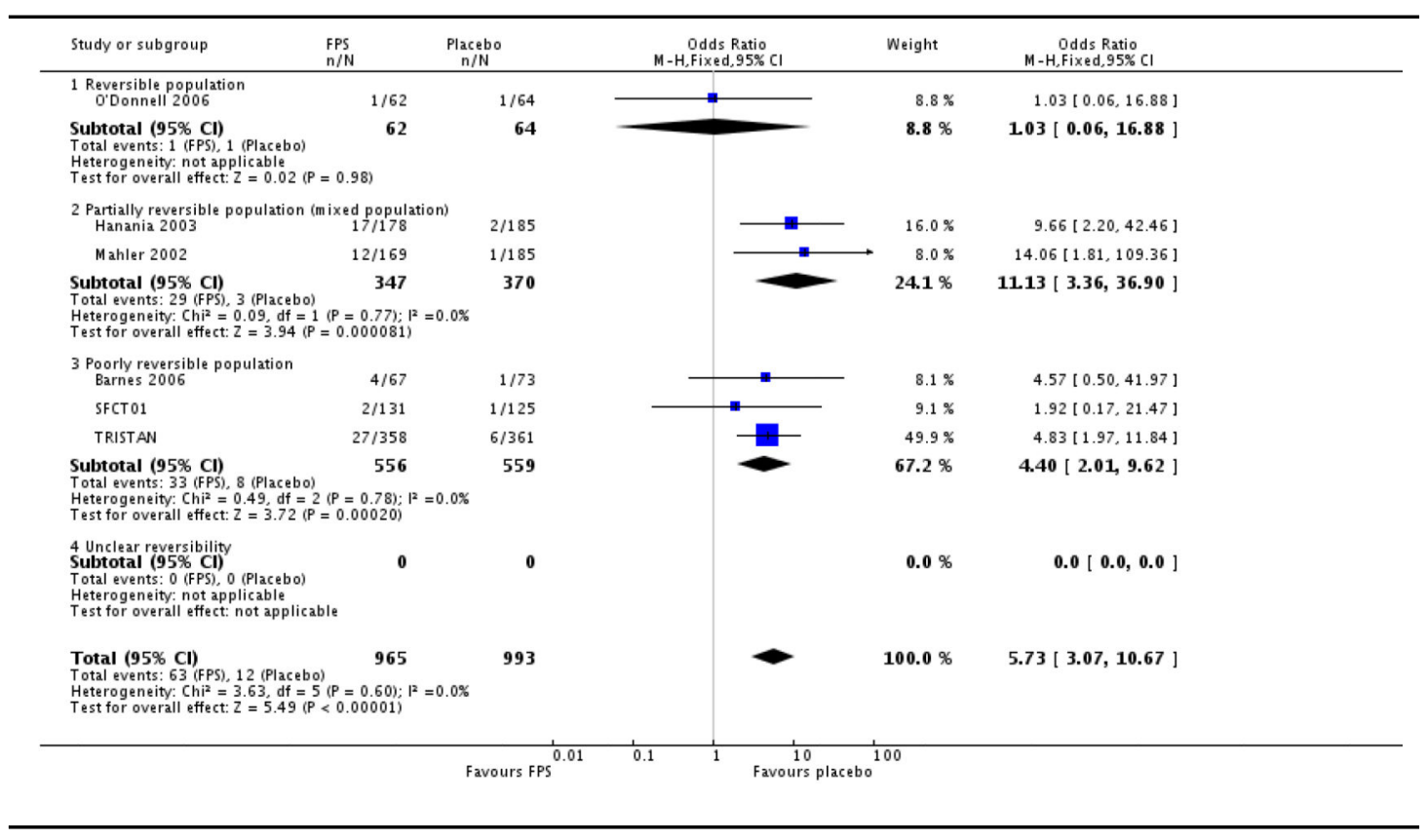


Analysis 2.26

Comparison 2 Fluticasone/salmeterol (FPS) versus placebo (PLA), Outcome 26 Withdrawals due to adverse events

Review: Com bine d corticosteroid and long-acting beta-agonist in one inhaler versus placebo for chronic obstructive pulmonary disease Comparison: 2 Fluticasone/salmeterol (FPS) versus placebo (PLA)

Outcome: 26 Withdrawals due to adverse events

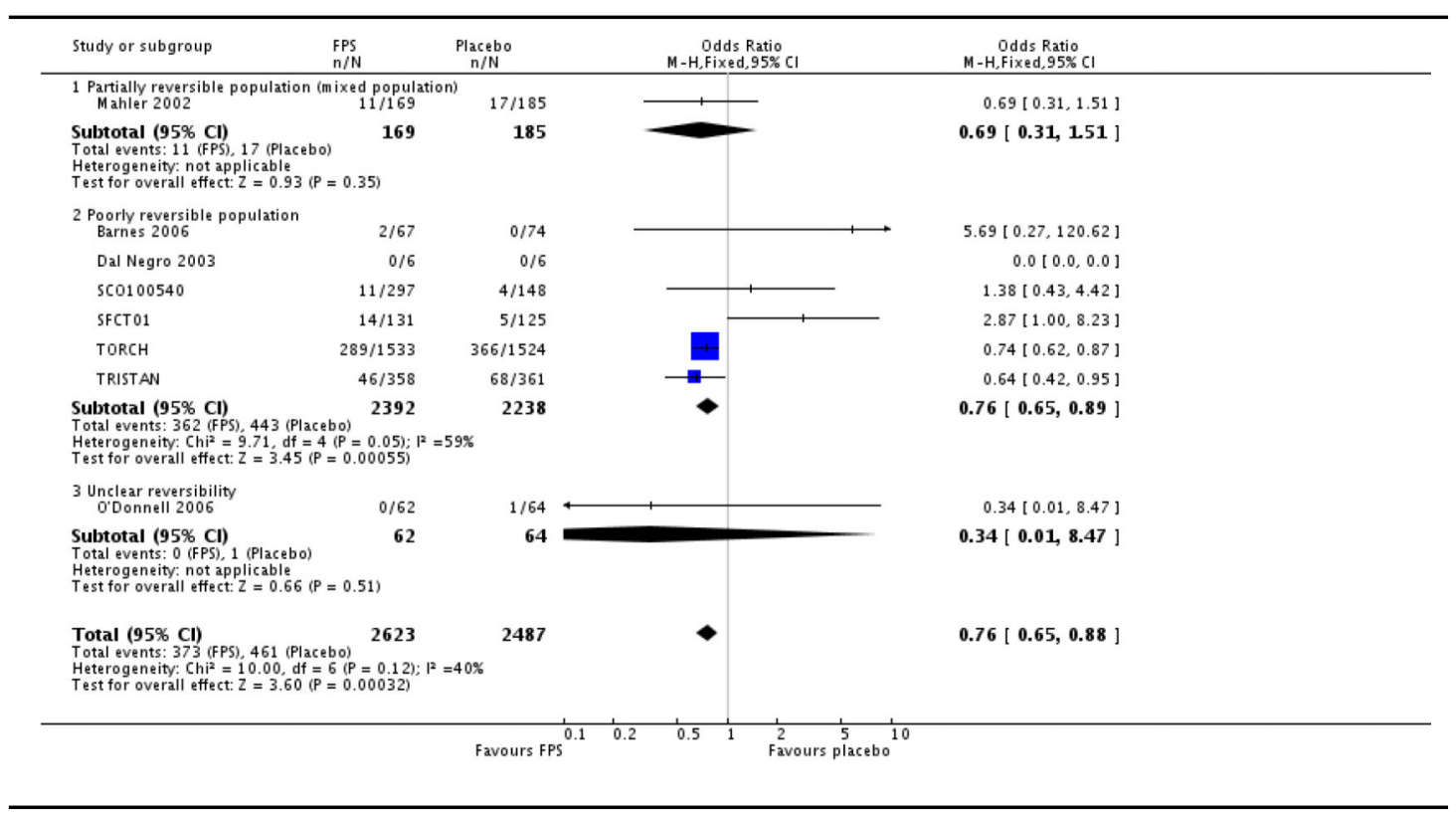


Analysis 2.27

Comparison 2 Fluticasone/salmeterol (FPS) versus placebo (PLA), Outcome 27 Withdrawals due to lack of efficacy/exacerbations

Review: Combined corticosteroid and long-acting beta-agonist in one inhaler versus placebo for chronic obstructive pulmonary disease Comparison: 2 Fluticasone/salmeterol (FPS) versus placebo (PLA)

Outcome: 27 Withdrawals due to lack of efficacy/exacerbations

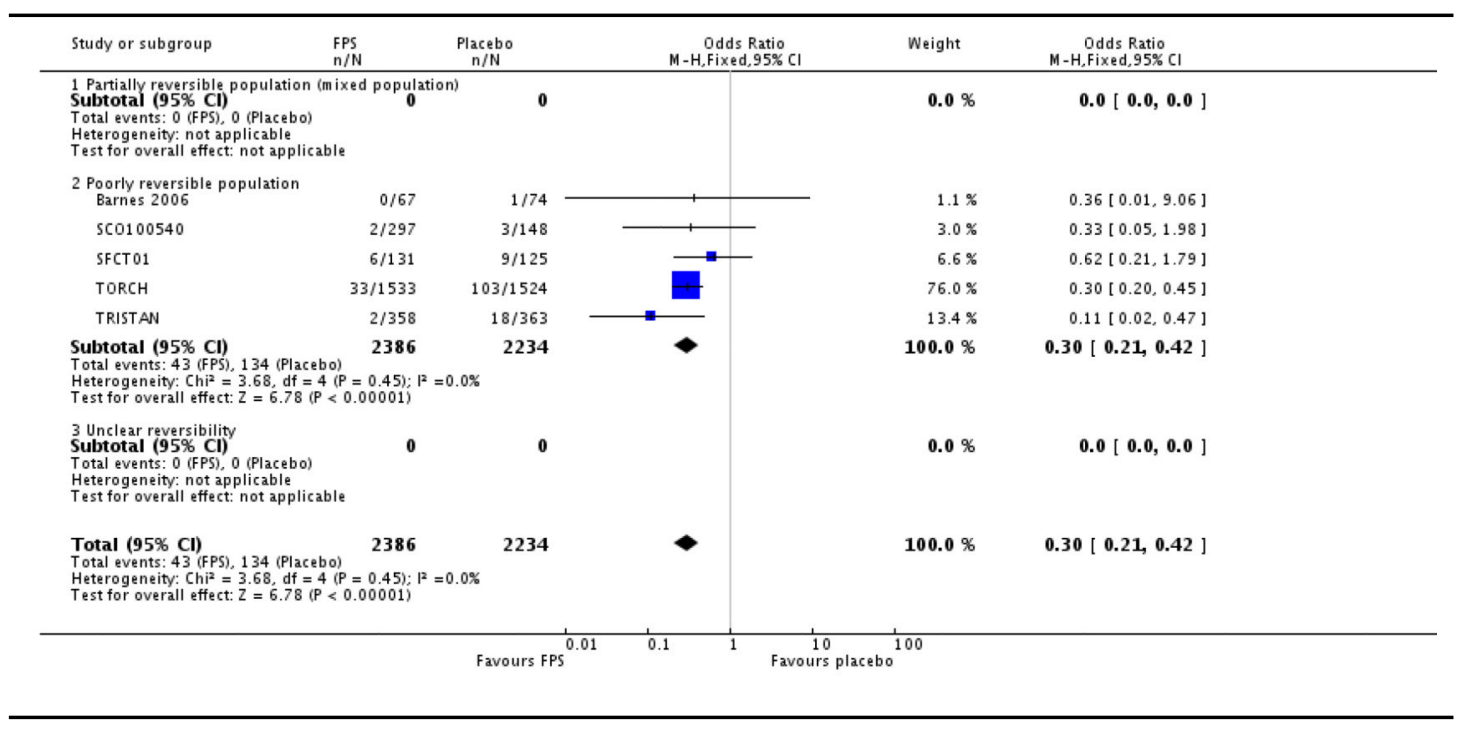




\section{Analysis 2.28}

\section{Comparison 2 Fluticasone/salmeterol (FPS) versus placebo (PLA), Outcome 28 Withdrawals}

Review: Combined corticosteroid and long-acting beta-agonist in one inhaler versus placebo for chronic obstructive pulmonary disease Comparison: 2 Fluticasone/salmeterol (FPS) versus placebo (PLA)

Outcome: 28 Withdrawals

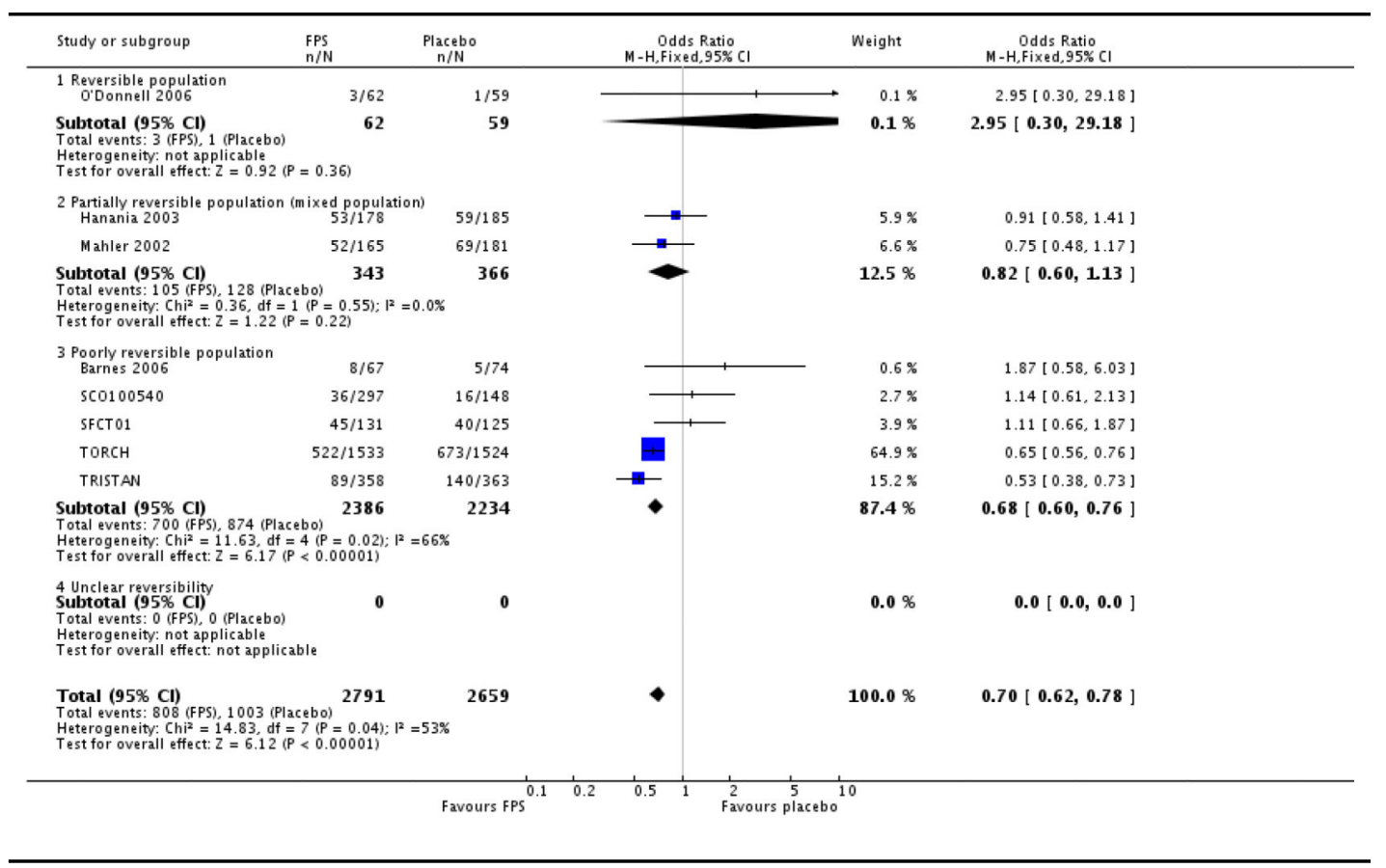




\section{Analysis 2.29}

\section{Comparison 2 Fluticasone/salmeterol (FPS) versus placebo (PLA), Outcome 29 Withdrawals due to adverse events - pneumonia}

Review: Combined corticosteroid and long-acting beta-agonist in one inhaler versus placebo for chronic obstructive pulmonary disease Comparison: 2 Fluticasone/salmeterol (FPS) versus placebo (PLA)

Outcome: 29 Withdrawals due to adverse events - pneumonia

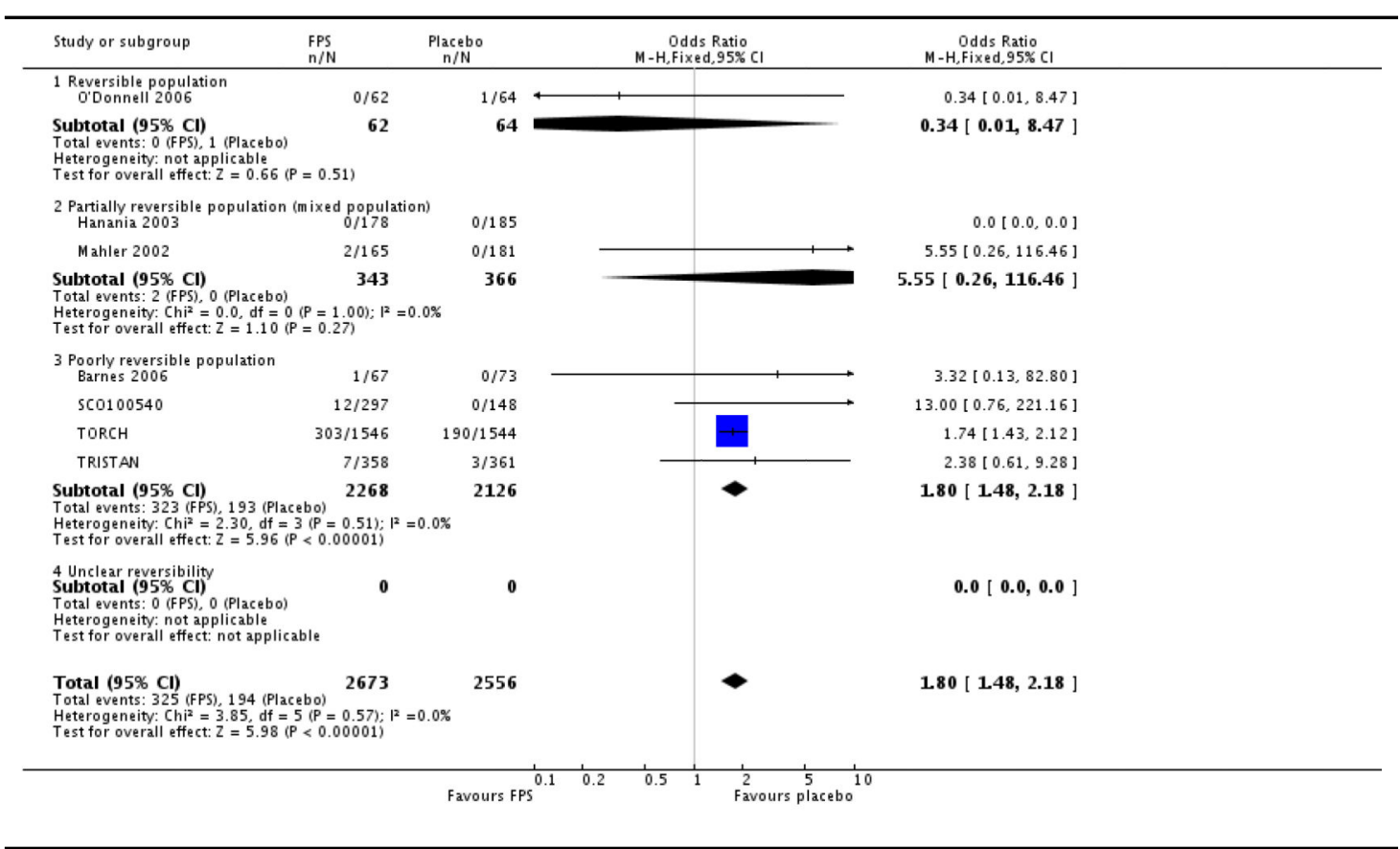


Analysis 2.30

Comparison 2 Fluticasone/salmeterol (FPS) versus

placebo (PLA), Outcome 30 Adverse event nasopharyngitis

Review: Combined corticosteroid and long-acting beta-agonist in one inhaler versus placebo for chronic obstructive pulmonary disease Comparison: 2 Fluticasone/salmeterol (FPS) versus placebo (PLA)

Outcome: 30 Adverse event - nasopharyngitis

\begin{tabular}{|c|c|c|c|c|}
\hline $\begin{array}{l}1 \text { Partially reversible population (mixed population) } \\
\text { Subtotal }(95 \% \text { CI) } \\
\text { Total events: } 0 \text { (FPS), (Placebo) } \\
\text { Heterogeneity: not applicable } \\
\text { Test for overall effect: not applicable }\end{array}$ & 0 & & $0.0 \%$ & $0.0[0.0,0.0]$ \\
\hline $\begin{array}{l}2 \text { Poorly reversible population } \\
\text { Sc0100540 }\end{array}$ & $28 / 148$ & & $17.8 \%$ & $0.93[0.56,1.55]$ \\
\hline $215 / 1546$ & $165 / 1544$ & + & $82.2 \%$ & $1.35[1.09,1.68]$ \\
\hline $\begin{array}{l}3 \text { Unclear reversibility } \\
\text { Subtotal }(95 \% \text { CI) } \\
\text { Total events: 0 (FPS), } 0 \text { (Placebo) } \\
\text { Heterogeneity: not app plicable } \\
\text { Test for overall effect: not applicable }\end{array}$ & 0 & & $0.0 \%$ & $0.0[0.0,0.0]$ \\
\hline $\begin{array}{l}\text { Total }(95 \% \mathrm{Cl}) \\
\text { Total events: } 268 \text { (FP) } 193(\mathrm{Placebo}) \quad \mathbf{1 8 4 3} \\
\text { Heterogeneity Chi }{ }^{2}=1.74, \mathrm{dt}=1(\mathrm{P}=0.19) ;\left.\right|^{2}=43 \% \\
\text { Test for overall effect: } z=2.39(\mathrm{P}=0.017)\end{array}$ & 3\% & $<$ & $100.0 \%$ & $1.28[1.05,156]$ \\
\hline
\end{tabular}

\section{Analysis 2.31}

Comparison 2 Fluticasone/salmeterol (FPS) versus

placebo (PLA), Outcome 31 Adverse events pharyngolaryngeal pain

Review: Combined corticosteroid and long-acting beta-agonist in one inhaler versus placebo for chronic obstructive pulmonary disease

Comparison: 2 Fluticasone/salmeterol (FPS) versus placebo (PLA)

Outcome: 31 Adverse events - pharyngolaryngeal pain

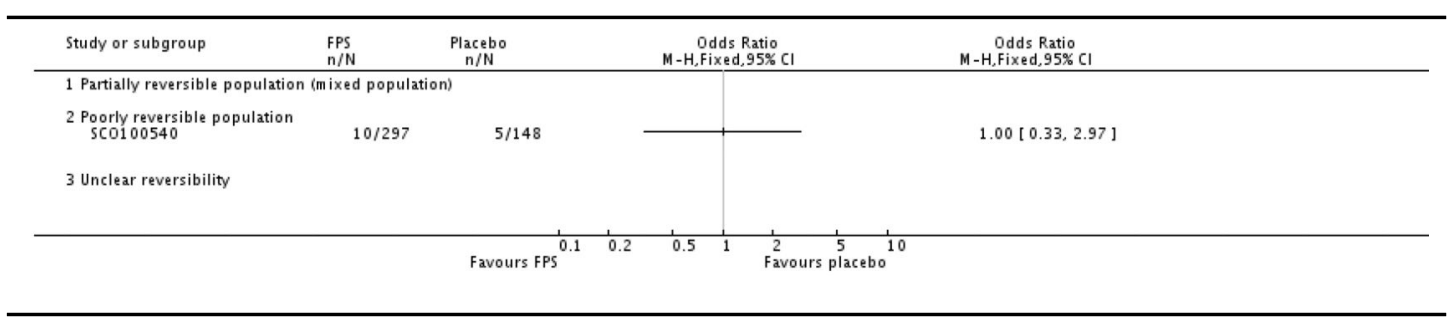


Analysis 2.32

\section{Comparison 2 Fluticasone/salmeterol (FPS) versus placebo (PLA), Outcome 32 Adverse events - upper respiratory tract infection}

Review: Combined corticosteroid and long-acting beta-agonist in one inhaler versus placebo for chronic obstructive pulmonary disease Comparison: 2 Fluticasone/salmeterol (FPS) versus placebo (PLA)

Outcome: 32 Adverse events - upper respiratory tract infection

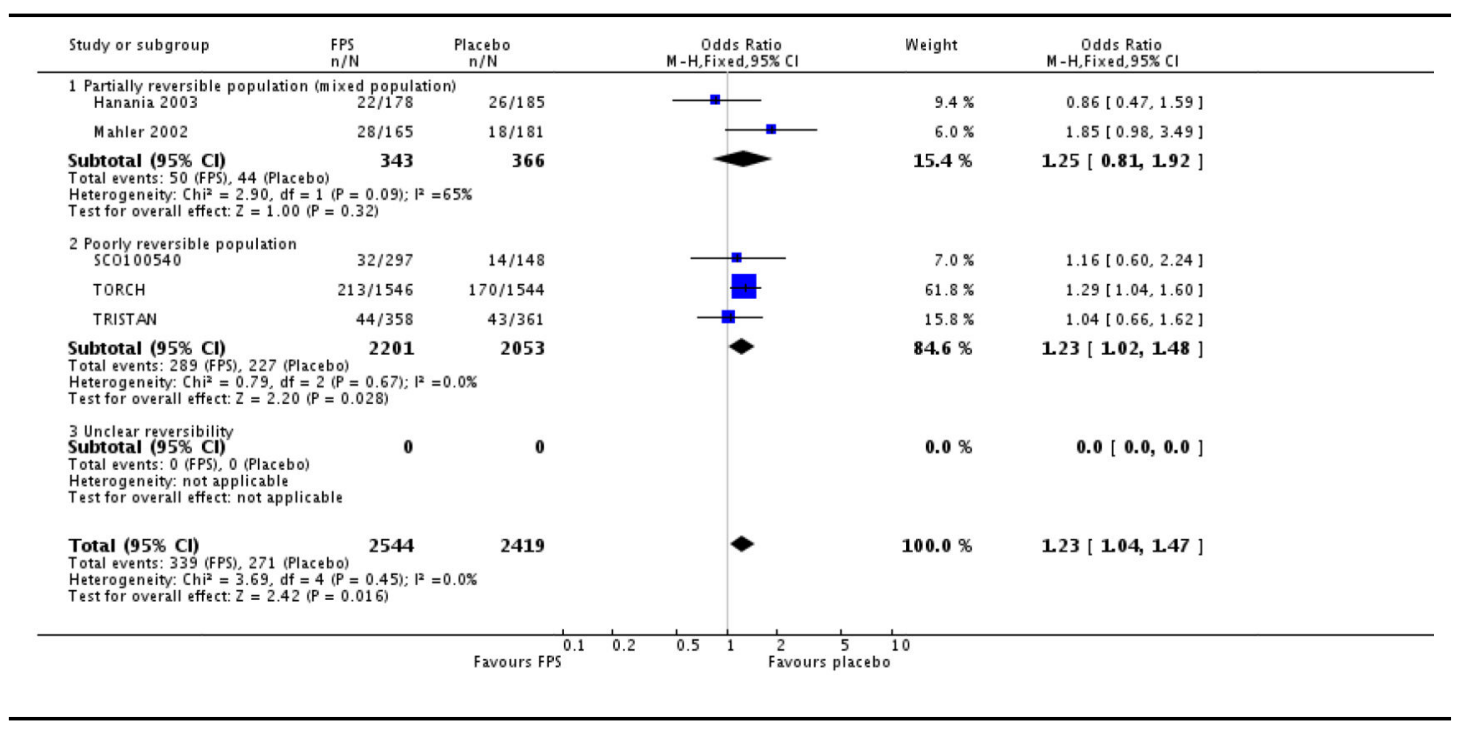


Analysis 2.33

\section{Comparison 2 Fluticasone/salmeterol (FPS) versus placebo (PLA), Outcome 33 Adverse events - headache}

Review: Combined corticosteroid and long-acting beta-agonist in one inhaler versus placebo for chronic obstructive pulmonary disease Comparison: 2 Fluticasone/salmeterol (FPS) versus placebo (PLA)

Outcome: 33 Adverse events - headache

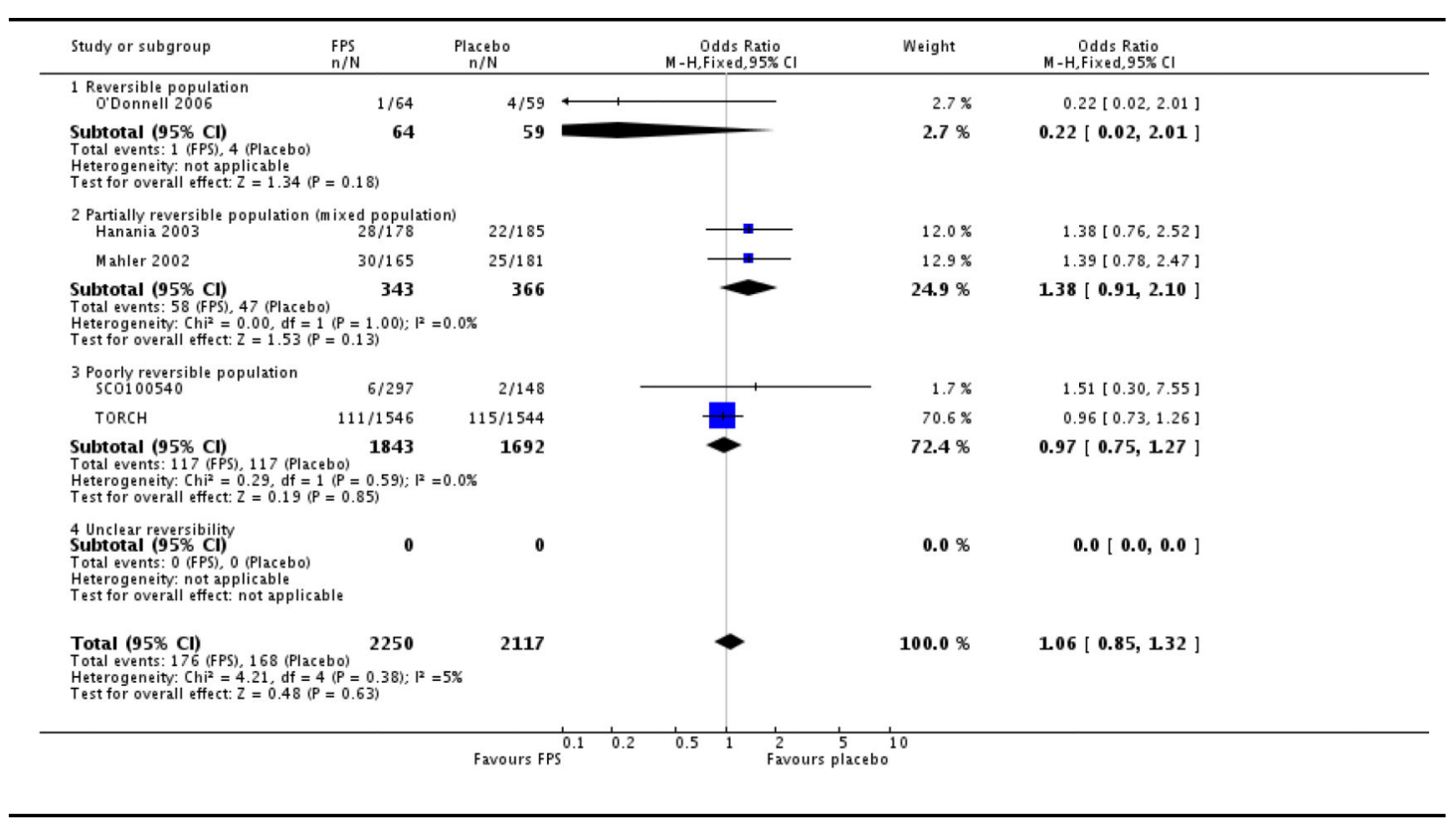




\section{Analysis 2.34}

Comparison 2 Fluticasone/salmeterol (FPS) versus placebo (PLA), Outcome 34 Adverse events - hoarseness

Review: Combined corticosteroid and long-acting beta-agonist in one inhaler versus placebo for chronic obstructive pulmonary disease Comparison: 2 Fluticasone/salmeterol (FPS) versus placebo (PLA)

Outcome: 34 Adverse events - hoarseness

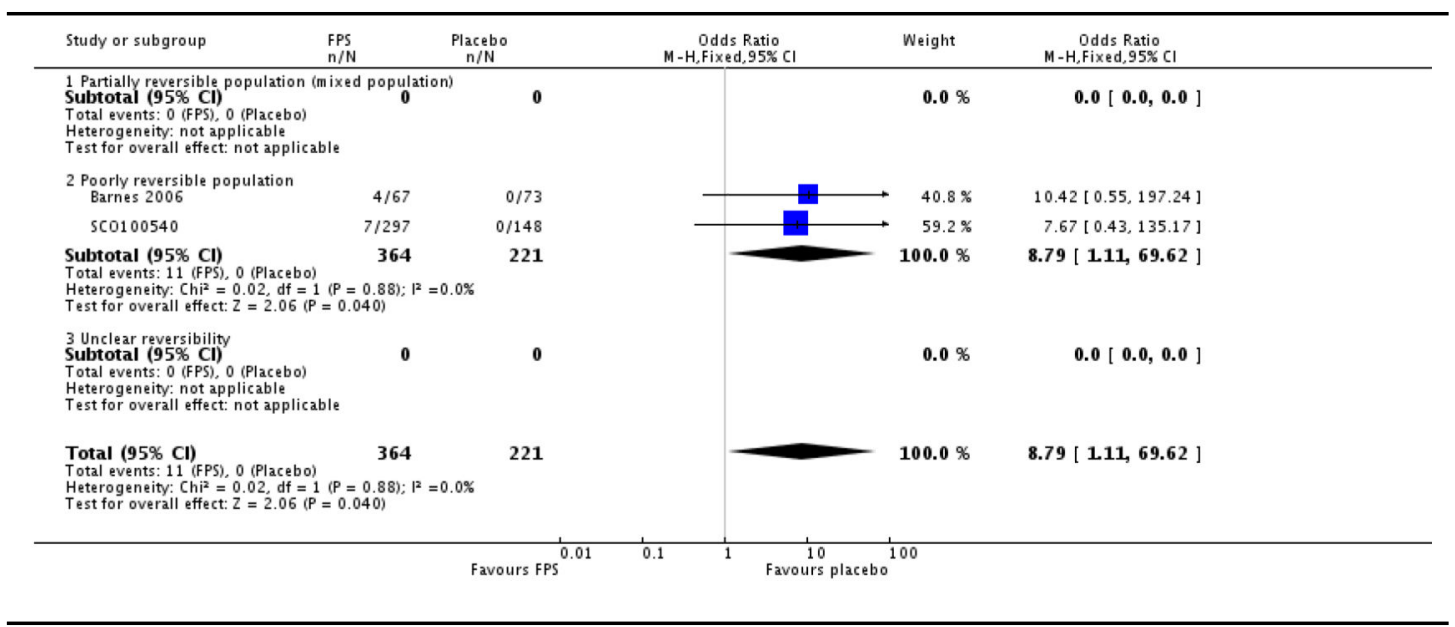

Analysis 2.35

Comparison 2 Fluticasone/salmeterol (FPS) versus placebo (PLA), Outcome 35 Adverse events - pyrexia

Review: Combined corticosteroid and long-acting beta-agonist in one inhaler versus placebo for chronic obstructive pulm onary disease Comparison: 2 Fluticasone/salmeterol (FPS) versus placebo (PLA) Outcome: 35 Adverse events - pyrexia

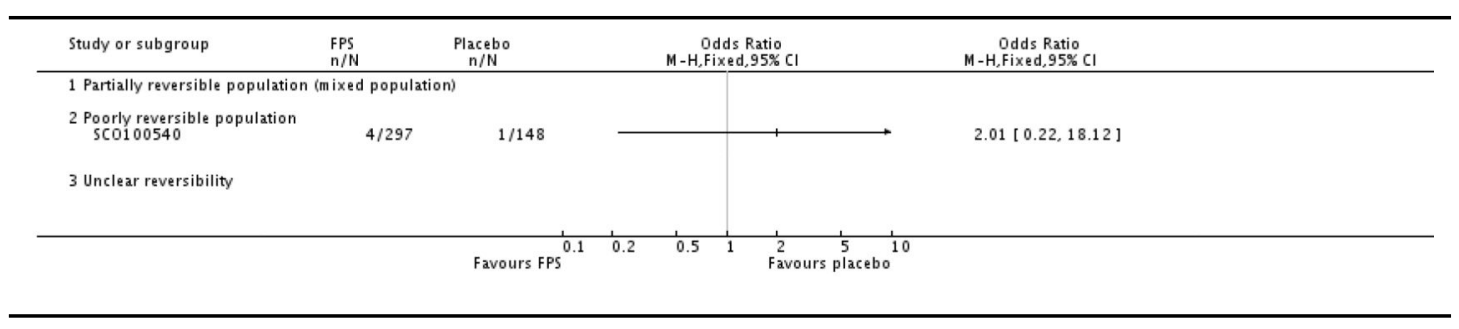




\section{Analysis 2.36}

\section{Comparison 2 Fluticasone/salmeterol (FPS) versus placebo (PLA), Outcome 36 Adverse events - cough}

Review: Combined corticosteroid and long-acting beta-agonist in one inhaler versus placebo for chronic obstructive pulmonary disease Comparison: 2 Fluticasone/salmeterol (FPS) versus placebo (PLA)

Outcome: 36 Adverse events - cough

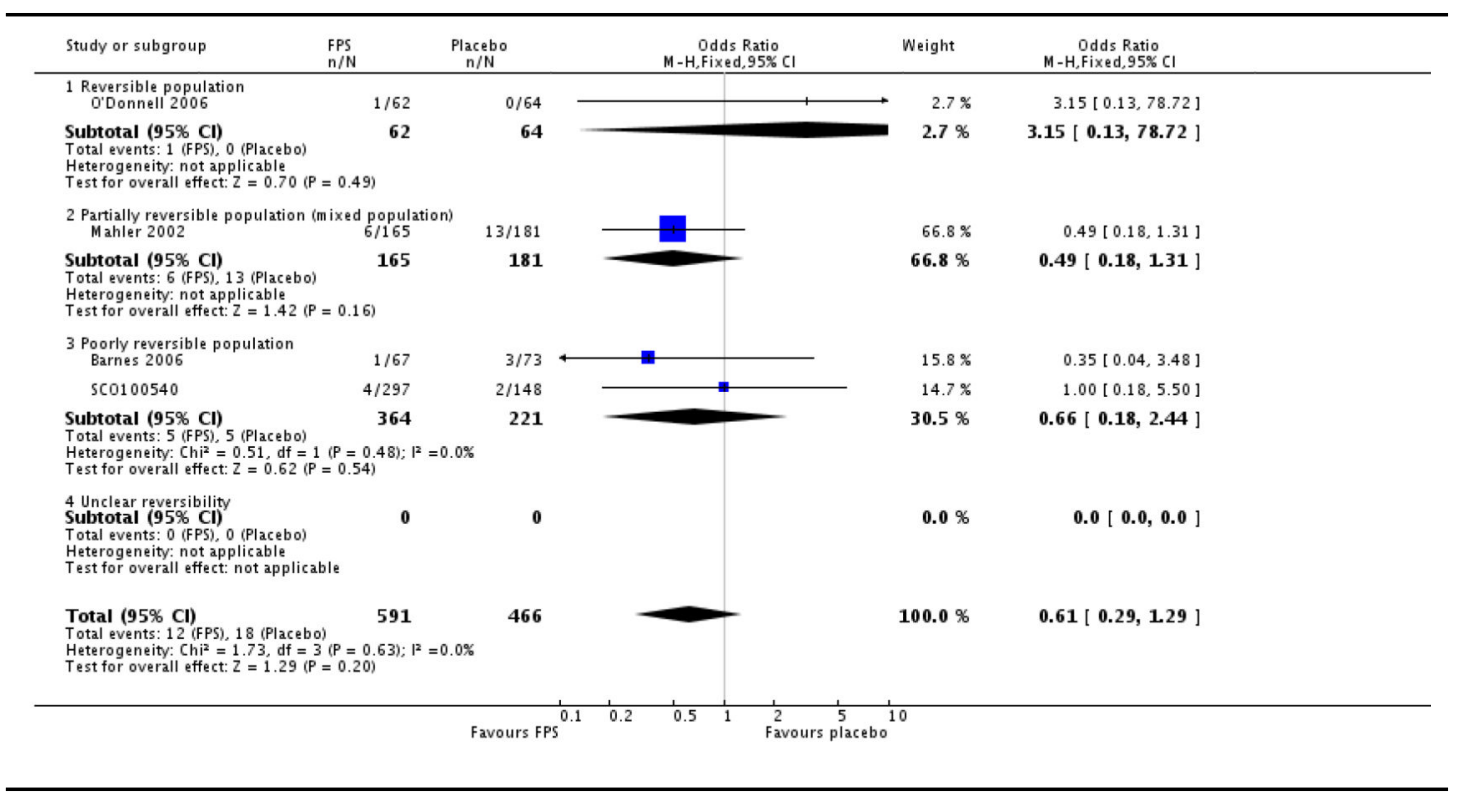

Analysis 2.37

Comparison 2 Fluticasone/salmeterol (FPS) versus placebo (PLA), Outcome 37 Adverse events palpitations

Review: Combined corticosteroid and long-acting beta-agonist in one inhaler versus placebo for chronic obstructive pulmonary disease Comparison: 2 Fluticasone/salmeterol (FPS) versus placebo (PLA)

Outcome: 37 Adverse events - palpitations

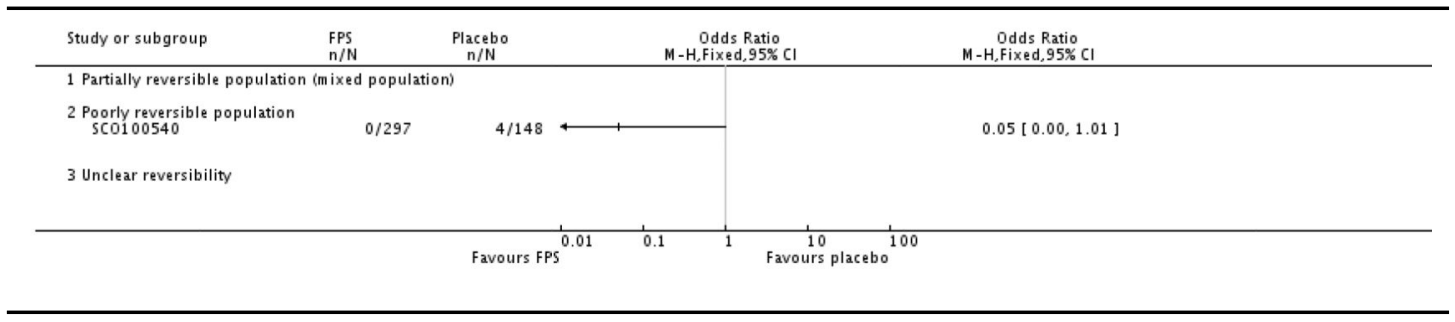


Analysis 2.38

Comparison 2 Fluticasone/salmeterol (FPS) versus placebo (PLA), Outcome 38 Adverse events - mouth ulceration

Review: Combined corticosteroid and long-acting beta-agonist in one inhaler versus placebo for chronic obstructive pulmonary disease Comparison: 2 Fluticasone/salmeterol (FPS) versus placebo (PLA)

Outcome: 38 Adverse events - mouth ulceration

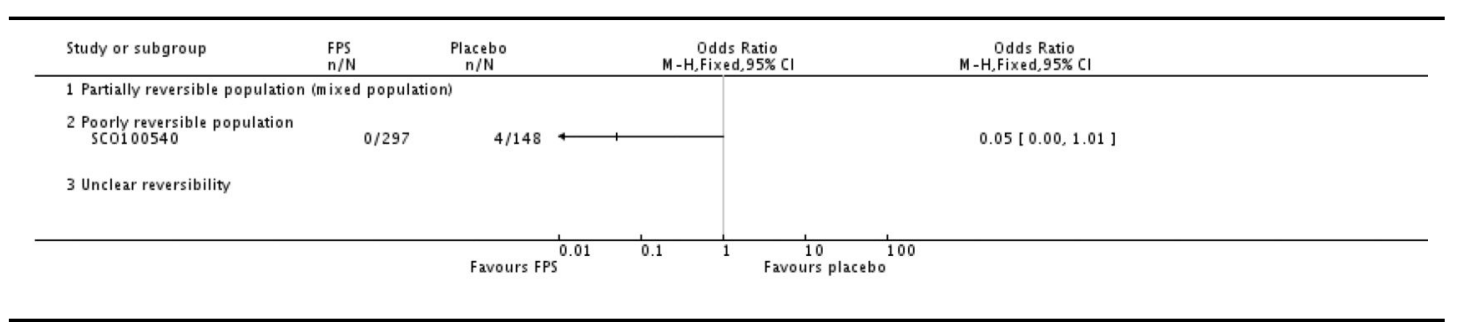

Analysis 2.39

omparison 2 Fluticasone/salmeterol (FPS) versus placebo (PLA), Outcome 39 Adverse events - toothache

Review: Combined corticosteroid and long-acting beta-agonist in one inhaler versus placebo for chronic obstructive pulmonary disease Comparison: 2 Fluticasone/salmeterol (FPS) versus placebo (PLA)

Outcome: 39 Adverse events - toothache

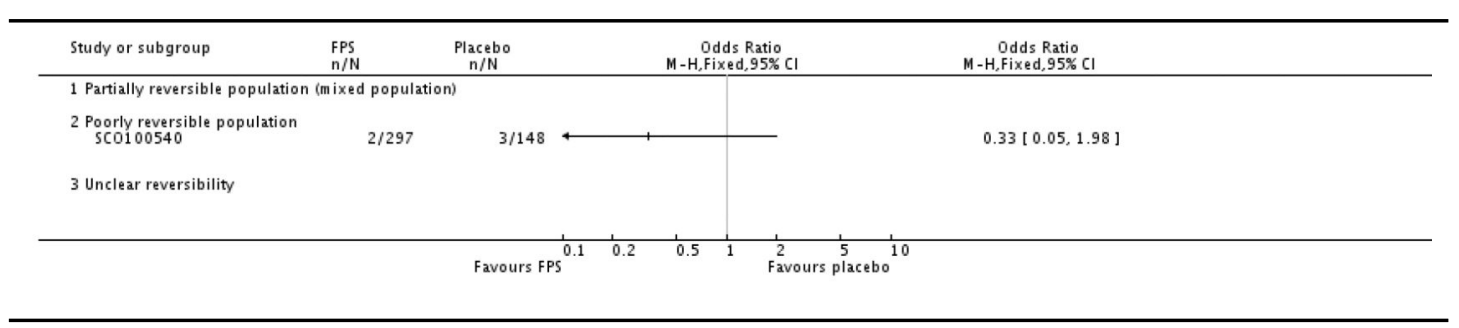


Analysis 2.40

Comparison 2 Fluticasone/salmeterol (FPS) versus placebo (PLA), Outcome 40 Adverse events - urinary tract infection

Review: Combined corticosteroid and long-acting beta-agonist in one inhaler versus placebo for chronic obstructive pulmonary disease Comparison: 2 Fluticasone/salmeterol (FPS) versus placebo (PLA)

Outcome: 40 Adverse events - urinary tract infection

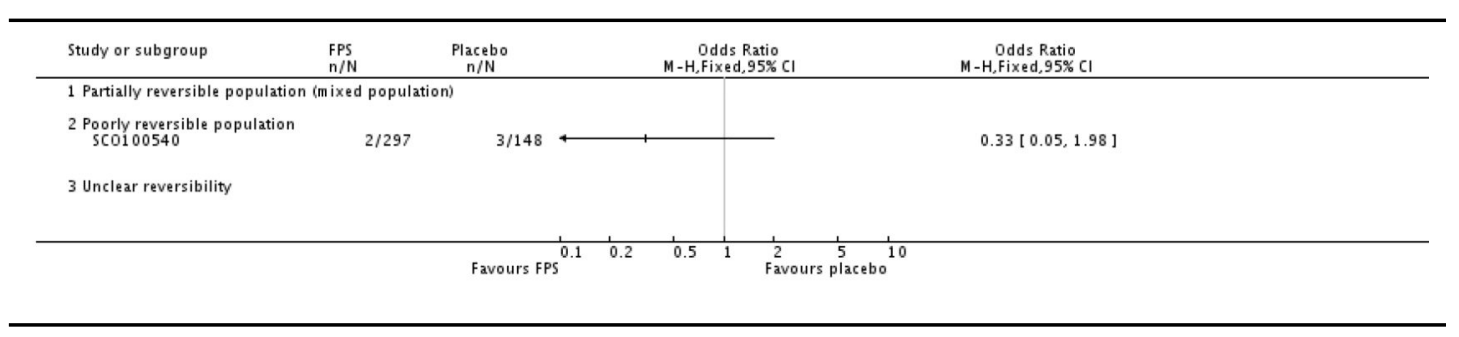

\section{Analysis 2.41}

Comparison 2 Fluticasone/salmeterol (FPS) versus placebo (PLA), Outcome 41 Adverse events - dyspnoea

Review: Combined corticosteroid and long-acting beta-agonist in one inhaler versus placebo for chronic obstructive pulmonary disease Comparison: 2 Fluticasone/salmeterol (FPS) versus placebo (PLA)

Outcome: 41 Adverse events - dyspnoea

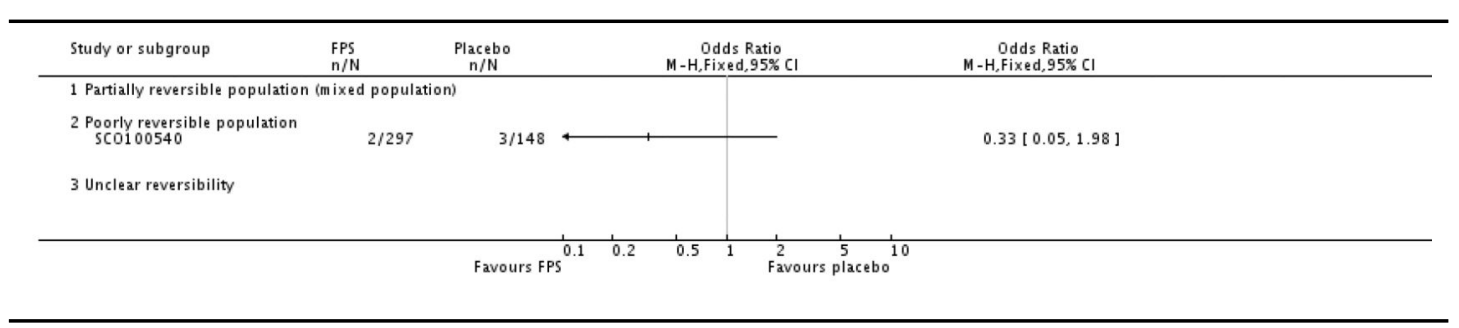


Analysis 2.42

Comparison 2 Fluticasone/salmeterol (FPS) versus placebo (PLA), Outcome 42 Adverse events - blood glucose increased

Review: Combined corticosteroid and long-acting beta-agonist in one inhaler versus placebo for chronic obstructive pulmonary disease Comparison: 2 Fluticasone/salmeterol (FPS) versus placebo (PLA)

Outcome: 42 Adverse events - blood glucose increased

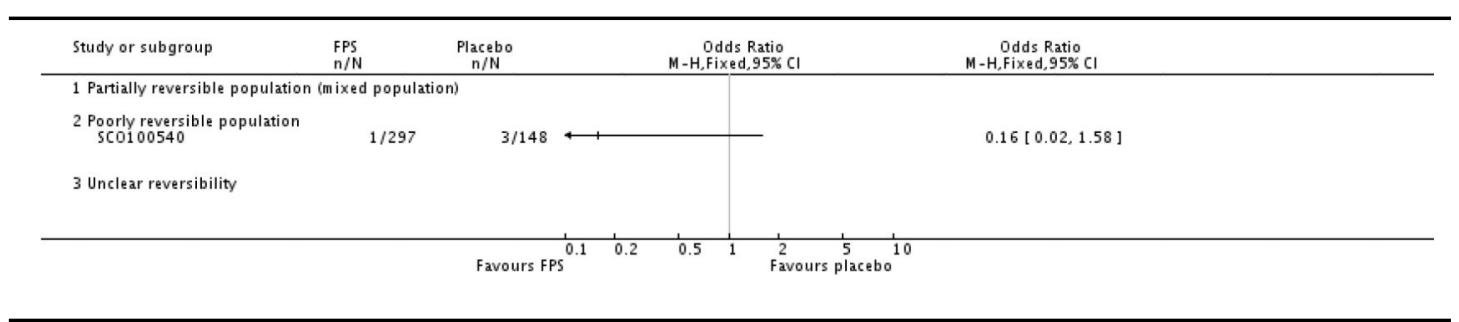

Analysis 2.43

Comparison 2 Fluticasone/salmeterol (FPS) versus placebo (PLA), Outcome 43 Adverse events - insomnia

Review: Combined corticosteroid and long-acting beta-agonist in one inhaler versus placebo for chronic obstructive pulmonary disease Comparison: 2 Fluticasone/salmeterol (FPS) versus placebo (PLA)

Outcome: 43 Adverse events - insomnia

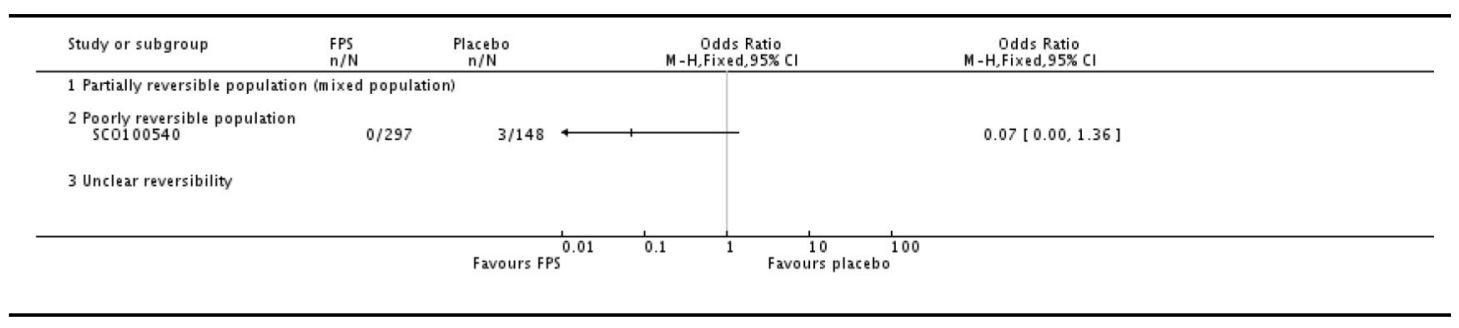


Analysis 2.44

Comparison 2 Fluticasone/salmeterol (FPS) versus placebo (PLA), Outcome 44 Adverse events - bronchitis

Review: Combined corticosteroid and long-acting beta-agonist in one inhaler versus placebo for chronic obstructive pulmonary disease Comparison: 2 Fluticasone/salmeterol (FPS) versus placebo (PLA)

Outcome: 44 Adverse events - bronchitis

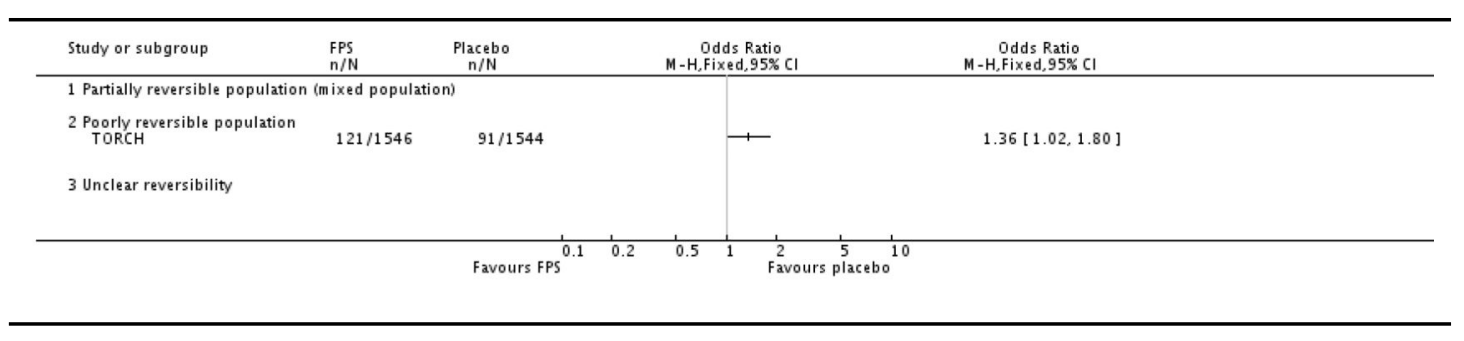

\section{Analysis 3.1}

Comparison 3 Budesonide/formoterol (BDF) versus placebo (PLA), Outcome 1 Severe Exacerbations

Review: Combined corticosteroid and long-acting beta-agonist in one inhaler versus placebo for chronic obstructive pulmonary disease

Comparison: 3 Budesonide/formoterol (BDF) versus placebo (PLA)

Outcome: 1 Severe Exacerbations

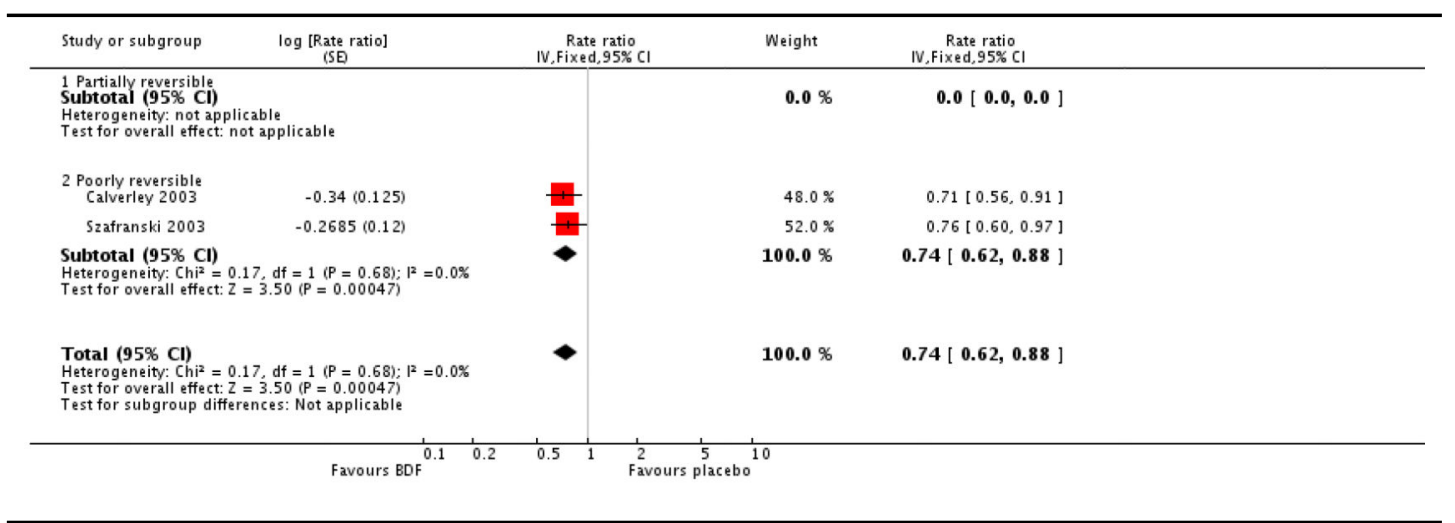


Analysis 3.2

\section{Comparison 3 Budesonide/formoterol (BDF) versus placebo (PLA), Outcome 2 Mean severe exacerbation rates per patient per year}

Review: Combined corticosteroid and long-acting beta-agonist in one inhaler versus placebo for chronic obstructive pulmonary disease Comparison: 3 Budesonide/formoterol (BDF) versus placebo (PLA)

Outcome: 2 Mean severe exacerbation rates per patient per year

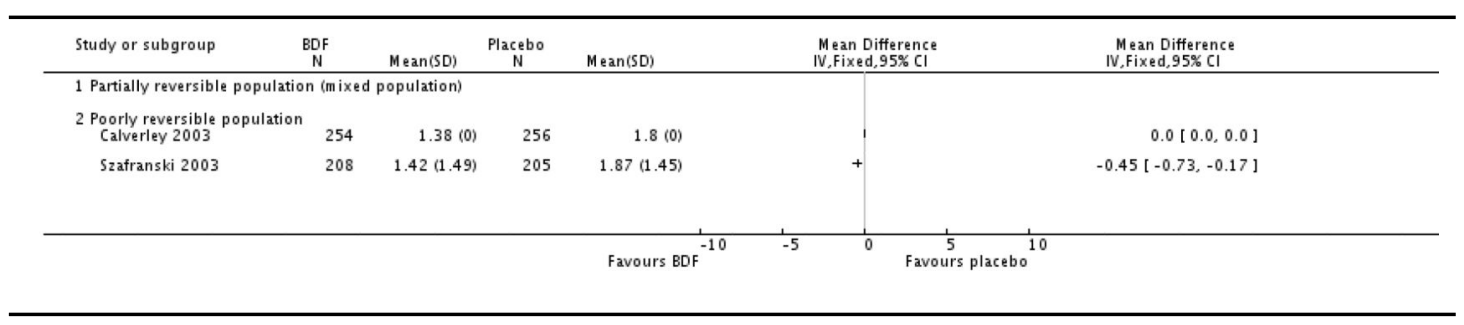

\section{Analysis 3.3}

Comparison 3 Budesonide/formoterol (BDF) versus placebo (PLA), Outcome 3 Quality of life - SGRQ (change scores)

Review: Combined coitico steroid and long-acting beta-agonist in one inhaler versus placebo for chronic obstructive pulmonary disease Comparison: 3 Budesonide/formoterol (BDF) versus placebo (PLA)

Outcome: 3 duality of life - SGRQ (change scores)

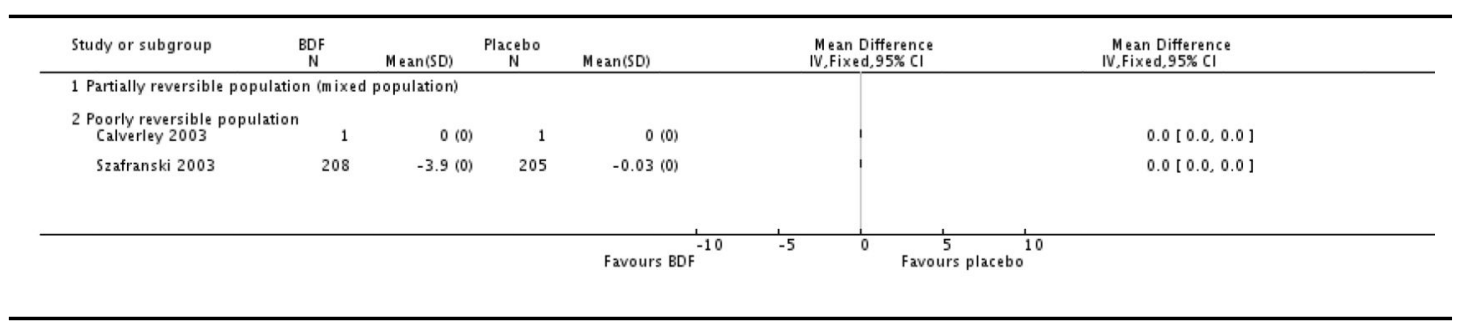




\section{Analysis 3.4}

\section{Comparison 3 Budesonide/formoterol (BDF) versus placebo (PLA), Outcome 4 Quality of life - change scores}

Review: Combined corticosteroid and long-acting beta-agonist in one inhaler versus placebo for chronic obstructive pulmonary disease Comparison: 3 Budesonide/form oterol (BDF) versus placebo (PLA)

Outcome: 4 Quality of life - change scores

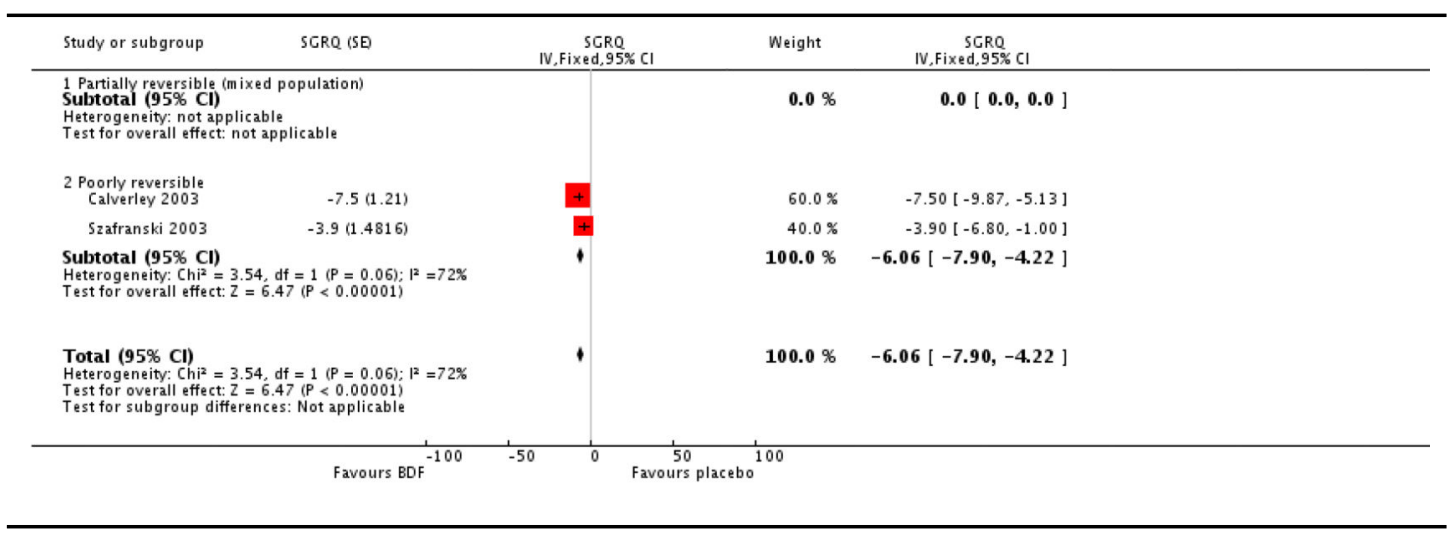

Analysis 3.5

Comparison 3 Budesonide/formoterol (BDF) versus placebo (PLA), Outcome 5 Rescue medication usage

Review: Combined corticosteroid and long-acting beta-agonist in one inhaler versus placebo for chronic obstructive pulmonary disease Comparison: 3 Budesonide/formoterol (BDF) versus placebo (PLA)

Outcome: 5 Rescue medication usage

\begin{tabular}{|c|c|c|c|c|}
\hline Study or subgroup & Puffs per day (SE) & $\begin{array}{r}\text { Puffs per day } \\
\text { IV, Fixed, } 95 \% \mathrm{CI}\end{array}$ & Weight & $\begin{array}{l}\text { Puffs per day } \\
\text { IV. Fixed, } 95 \% \mathrm{CI}\end{array}$ \\
\hline \multicolumn{2}{|c|}{$\begin{array}{l}\text { 1 Partially reversible (mixed population) } \\
\text { Subtotal ( }(95 \% \mathrm{Cl}) \\
\text { Heterogeneity, not applicable } \\
\text { Test for overall effect: not applicable }\end{array}$} & & $0.0 \%$ & $0.0[0.0,0.0]$ \\
\hline \multicolumn{2}{|l|}{2 Poorly reversible } & + & $86.3 \%$ & $-0.80[-1.11,-0.49]$ \\
\hline Szafranski 2003 & $-1.3(0.3911)$ & $\#$ & $13.7 \%$ & $-1.30[-2.07,-0.53]$ \\
\hline \multicolumn{2}{|c|}{$\begin{array}{l}\text { Subtotal }(95 \% \mathrm{Cl}) \\
\text { Heterogeneity: } \mathrm{Chi} \mathrm{i}^{2}=1.41, \mathrm{df}=1(\mathrm{P}=0.24) ; \mathrm{l}^{2}=29 \% \\
\text { Test for overall effect: } Z=5.99(\mathrm{P}\langle 0.00001)\end{array}$} & - & $100.0 \%$ & $-0.87[-1.15,-0.58]$ \\
\hline \multicolumn{2}{|c|}{ 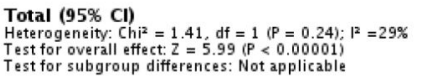 } & $\bullet$ & $100.0 \%$ & $-0.87[-1.15,-0.58]$ \\
\hline
\end{tabular}




\section{Analysis 3.6}

\section{Comparison 3 Budesonide/formoterol (BDF) versus placebo (PLA), Outcome 6 Symptoms (change scores)}

Review: Combined corticosteroid and long-acting beta-agonist in one inhaler versus placebo for chronic obstructive pulmonary disease Comparison: 3 Budesonide/formoterol (BDF) versus placebo (PLA)

Outcome: 6 Symptoms (change scores)

\begin{tabular}{|c|c|c|c|c|}
\hline Study or subgroup & Sym ptom scale (SE) & $\begin{array}{r}\text { Sym ptom scale } \\
\text { IV,Fixed, } 95 \% \mathrm{Cl}\end{array}$ & Weight & $\begin{array}{r}\text { Sym ptom scale } \\
\text { IV,Fixed, } 95 \% \mathrm{Cl}\end{array}$ \\
\hline \multicolumn{2}{|c|}{$\begin{array}{l}\text { 1 Partially reversible (mixed population) } \\
\text { Subtotal ( } 95 \% \text { Cl) } \\
\text { Heterogeneity not applicable } \\
\text { Test for overall effect: not applicable }\end{array}$} & & $0.0 \%$ & $0.0[0.0,0.0]$ \\
\hline \multicolumn{2}{|l|}{$\begin{array}{l}2 \text { Poorly reversible } \\
\text { Calverley } 2003\end{array}$} & & $66.1 \%$ & $-0.56[-0.88,-0.24]$ \\
\hline \multicolumn{2}{|l|}{ Szafranski 2003} & & $33.9 \%$ & $-0.77[-1.22,-0.32]$ \\
\hline \multicolumn{2}{|c|}{$\begin{array}{l}\text { Sulbtotal }(95 \% \mathrm{Cl}) \\
\text { Heterogeneity Chi' }=0.54, \mathrm{df}=1(P=0.46) ;\left.\right|^{2}=0.0 \% \\
\text { Test for overall effect: } Z=4.68(P<0.00001)\end{array}$} & & $100.0 \%$ & $-0.63[-0.90,-0.37]$ \\
\hline \multicolumn{2}{|c|}{$\begin{array}{l}\text { Total }(95 \% \mathrm{Cl}) \\
\text { Heterogeneity: Chi }=0.54, \text { df }=1(P=0.46) ; 1^{2}=0.0 \% \\
\text { Test for overall effectit } Z=4.68(P<0.00001) \\
\text { Test for subgroup differences: Not applicable }\end{array}$} & & $100.0 \%$ & $-0.63[-0.90,-0.37]$ \\
\hline
\end{tabular}

\section{Analysis 3.7}

Comparison 3 Budesonide/formoterol (BDF) versus placebo (PLA), Outcome 7 Mean FEV1 (\% change from baseline)

Review: Combined corticosteroid and long-acting beta-agonist in one inhaler versus placebo for chronic obstructive pulmonary disease Comparison: 3 Budesonide/formoterol (BDF) versus placebo (PLA) Outcome: 7 Mean FEV1 (\% change from baseline)

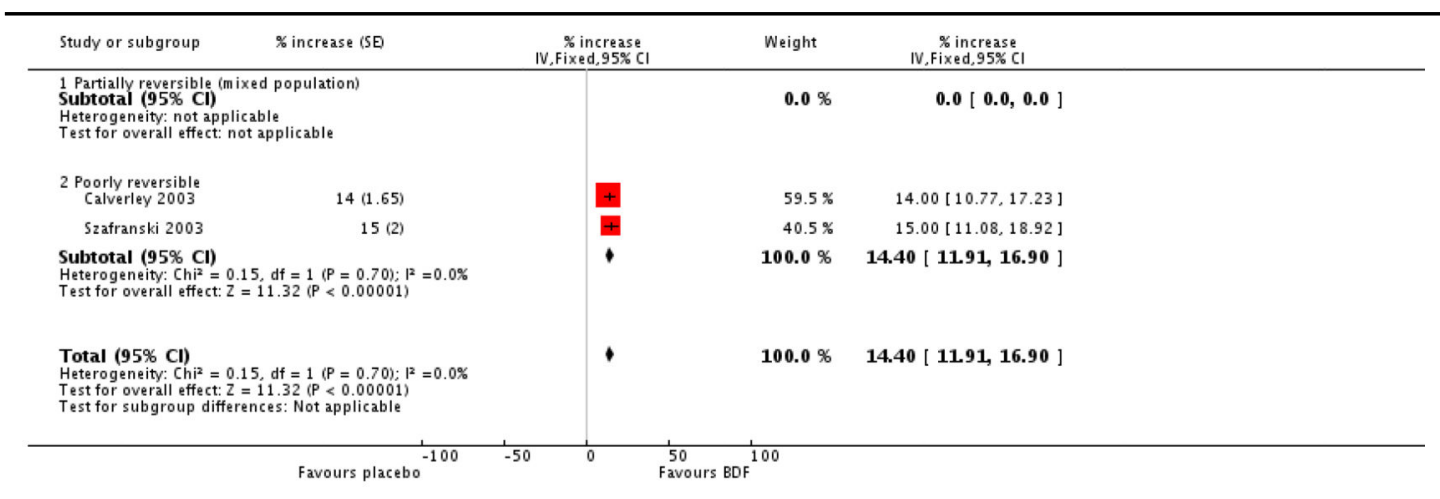




\section{Analysis 3.8}

\section{Comparison 3 Budesonide/formoterol (BDF) versus placebo (PLA), Outcome 8 Adverse events - 'serious events'}

Review: Combined corticosteroid and long-acting beta-agonist in one inhaler versus placebo for chronic obstructive pulmonary disease Comparison: 3 Budesonide/formoterol (BDF) versus placebo (PLA)

Outcome: 8 Adverse events - 'serious events'

\begin{tabular}{|c|c|c|c|c|}
\hline $\begin{array}{l}2 \text { Poorly reversible population } \\
\text { Calverley } 2003\end{array}$ & $66 / 256$ & & $62.3 \%$ & $0.99[0.67,1.47]$ \\
\hline Szafranski 2003 & $37 / 205$ & - & $37.7 \%$ & $1.18[0.73,1.93]$ \\
\hline \multicolumn{5}{|l|}{$\begin{array}{l}\text { Total events: } 108 \text { (BDF), } 103(\text { Placebo) } \\
\text { Heterogeneity: Chi }{ }^{2}=0.31, \mathrm{df}=1(\mathrm{P}=0.58) ; \mathrm{I}^{2}=0.0 \% \\
\text { Test for overall effect: } Z=0.39(\mathrm{P}=0.70)\end{array}$} \\
\hline & 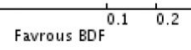 & $\begin{array}{l}1 \\
0.5\end{array}$ & ${ }_{\text {placebo }}^{1}{ }^{1}$ & \\
\hline
\end{tabular}

Analysis 3.10

Comparison 3 Budesonide/formoterol (BDF) versus placebo (PLA), Outcome 10 Adverse events pneumonia

Review: Combined corticosteroid and long-acting beta-agonist in one inhaler versus placebo for chronic obstructive pulmonary disease Comparison: 3 Budesonide/formoterol (BDF) versus placebo (PLA) Outcome: 10 Adverse events - pneumonia

\begin{tabular}{|c|c|c|c|c|c|}
\hline \multirow{2}{*}{$\begin{array}{c}\text { Study or subgroup } \\
\text { Calverley } 2003\end{array}$} & \multirow{2}{*}{$\begin{array}{l}\begin{array}{l}\mathrm{FPS} \\
\mathrm{n} / \mathrm{N}\end{array} \\
\\
8 / 254\end{array}$} & \multirow{2}{*}{$\begin{array}{r}\frac{\substack{\text { Placebo } \\
\mathrm{n} / \mathrm{N}}}{2 / 256} \\
\end{array}$} & $\begin{array}{c}\text { Odds Ratio } \\
M-\mathrm{H}, \mathrm{Fixed}, 95 \% \mathrm{Cl}\end{array}$ & \multirow{2}{*}{$\begin{array}{l}\text { Weight } \\
100.0 \%\end{array}$} & \multirow{2}{*}{$\begin{array}{c}\text { Odds Ratio } \\
\text { M-H,Fixed.95\% Cl } \\
4.13[0.87,19.64]\end{array}$} \\
\hline & & & & & \\
\hline
\end{tabular}


Analysis 3.11

\section{Comparison 3 Budesonide/formoterol (BDF) versus placebo (PLA), Outcome 11 Withdrawals due to worsening COPD symptoms}

Review: Combined corticosteroid and long-acting beta-agonist in one inhaler versus placebo for chronic obstructive pulmonary disease Comparison: 3 Budesonide/formoterol (BDF) versus placebo (PLA)

Outcome: 11 Withdrawals due to worsening COPD symptoms

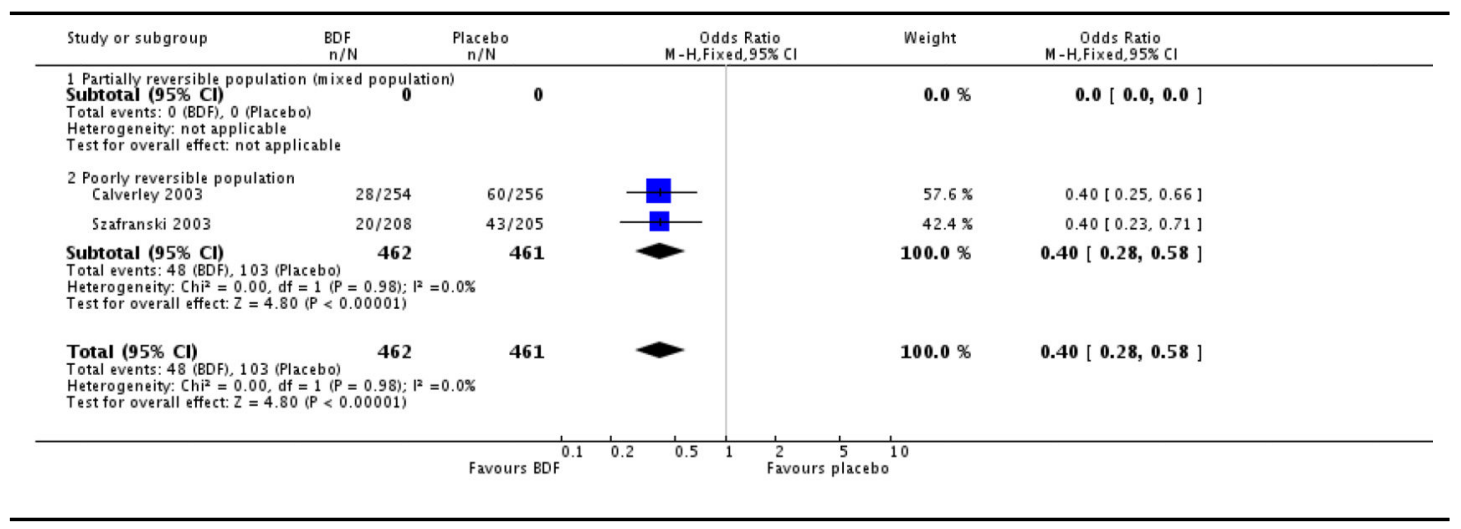

Analysis 3.12

Comparison 3 Budesonide/formoterol (BDF) versus placebo (PLA), Outcome 12 Withdrawals due to adverse events

Review: Combined corticosteroid and long-acting beta-agonist in one inhaler versus placebo for chronic obstructive pulmonary disease Comparison: 3 Budesonidi/form oterol (BDF) versus placebo (PLA)

Outcome: 12 Withdrawals due to adverse events

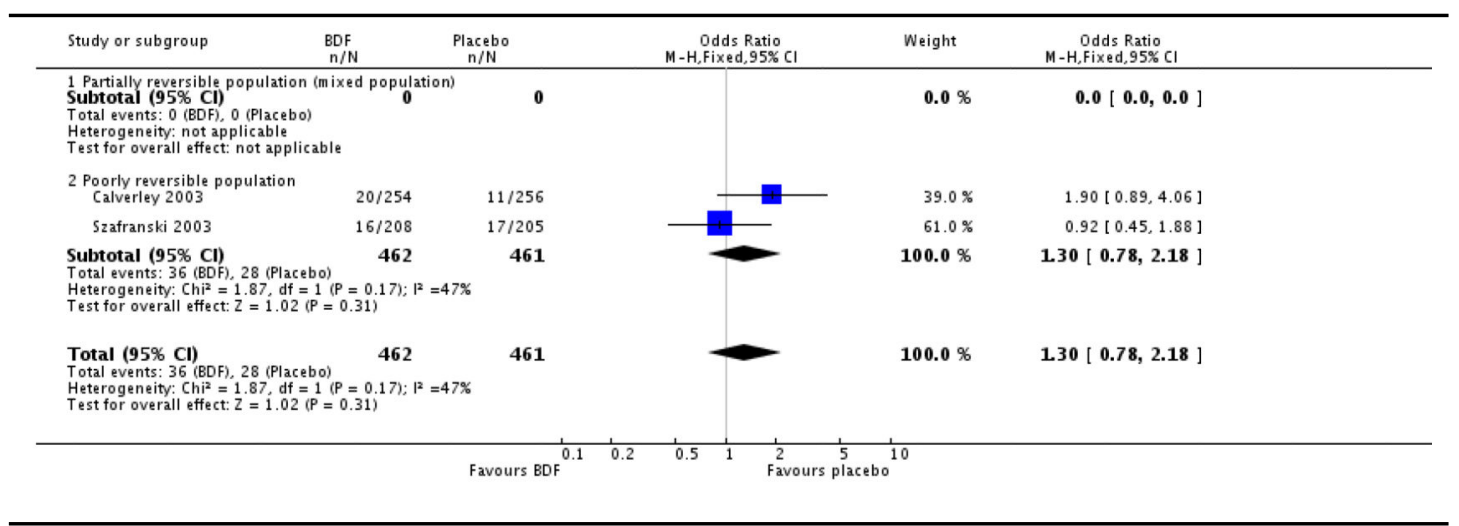




\section{Analysis 3.13}

\section{Comparison 3 Budesonide/formoterol (BDF) versus placebo (PLA), Outcome 13 Mortaity}

Review: Combined corticosteroid and long-acting beta-agonist in one inhaler versus placebo for chronic obstructive pulmonary disease Comparison: 3 Budesonide/form oterol (BDF) versus placebo (PLA)

Outcome: 13 Mortality

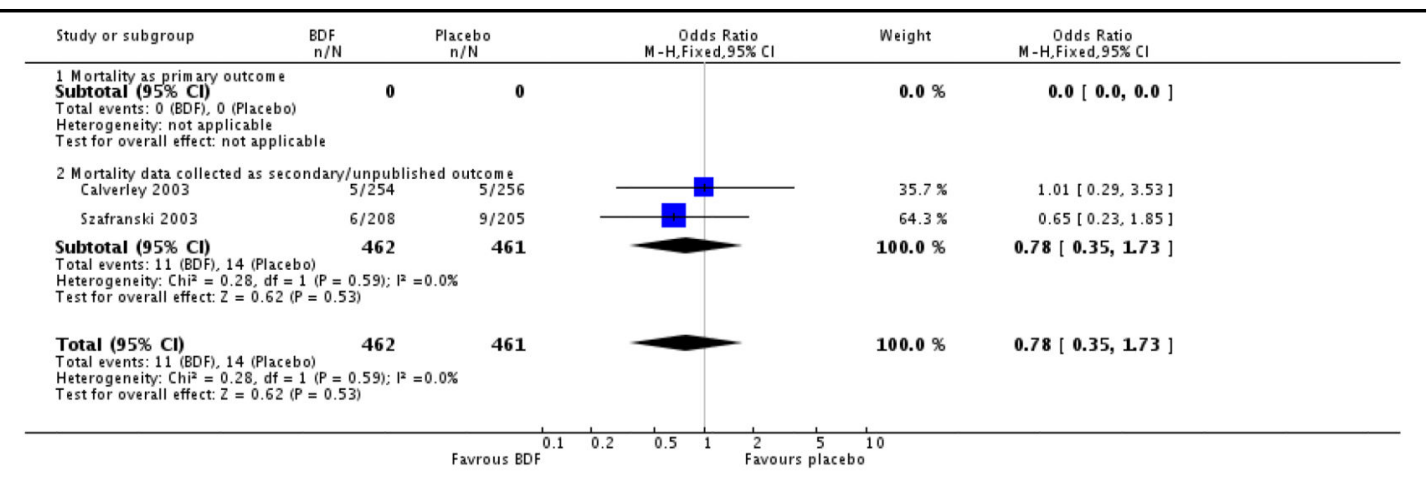

\section{WHAT'S NEW}

Last assessed as up-to-date: 1 August 2007.

\begin{tabular}{lll}
\hline Date & Event & Description \\
\hline 11 November 2009 & Amended & Spelling corrections and minor reformatting \\
\hline
\end{tabular}

\section{HISTORY}

Protocol first published: Issue 3, 2002

Review first published: Issue 4, 2003

\begin{tabular}{lll}
\hline Date & Event & Description \\
\hline 8 April 2008 & Amended & Converted to new review format. \\
\hline 26 February 2008 & Amended & $\begin{array}{l}\text { Summary of findings table now added to review prepared centrally in } \\
\text { GRADEpro by the Summary of Findings table working party (Nancy } \\
\text { Santesso) }\end{array}$ \\
\hline 2 August 2007 & $\begin{array}{l}\text { New citation } \\
\text { required and } \\
\text { conclusions } \\
\text { have changed }\end{array}$ & $\begin{array}{l}\text { Seven new studies met the entry criteria of the review (Barnes 2006; Kardos } \\
\text { 2007; TORCH; SCO100470; SCO40030; SFCT01; SCO10054). New } \\
\text { unpublished data have been incorporated for three studies previously included } \\
\text { (Hanania 2003; Mahler 2002; TRISTAN). } \\
\text { What was known before: Statistically significant findings in favour of } \\
\text { combination treatment over placebo. Conflicting findings when combination } \\
\text { treatment compared with monocompo-nent therapies. } \\
\text { What new data contribute to the review: }\end{array}$ \\
\hline
\end{tabular}




\begin{tabular}{lll}
\hline Date & Event & Description \\
\hline 30 April 2004 & $\begin{array}{l}\text { Data on all primary and secondary endpoints. Combined estimates now } \\
\text { indicate that combination fluticasone and salmeterol is significantly more } \\
\text { effective than fluticasone alone in reducing the rate of exacerbations }\end{array}$ \\
& $\begin{array}{l}\text { New citation } \\
\text { required and } \\
\text { conclusions } \\
\text { have changed }\end{array}$ & $\begin{array}{l}\text { Two new studies are included in this update (Calverly 2003; Hanania 2003). } \\
\text { One study previously reported in abstract form has now been published and } \\
\text { baseline and outcome data incorporated in this version of the review (Dal } \\
\text { Negro 2003). } \\
\text { Data on lung function have been pooled on a WMD rather than a SMD. Pooled } \\
\text { SEMs have been calculated from the published p values, and have been used to } \\
\text { calculate some exacerbation outcomes, as well as symptoms, quality of life and } \\
\text { lung function for some of the comparators. } \\
\text { The Discussion and Conclusion reflect the incorporation of the new data, and } \\
\text { the data calculated from previously published and included studies }\end{array}$ \\
& \\
&
\end{tabular}

\section{References to studies included in this review}

Barnes 2006 \{published and unpublished data\} . Barnes, NC.; Qiu, Y.; Pavord, I.; Parker, D.; Johnson, M.; Thompson, M., et al. Salmeterol /fluticasone propionate (SFC) anti-inflammatory effects in COPD [Abstract]; American Thoracic Society 2005 International Conference; San Diego, California. May 20-25; 2005. p. B93Poster 320

*Barnes NC, Qiu Y-S, Pavord ID, Parker D, Davis PA, Zhu J, et al. Antiinflammatory effects of salmeterol/fluticasone propionate in chronic obstructive lung disease. American Journal of Respiratory \& Critical Care Medicine. 2006; 173(7):736-43. [PubMed: 16424444]

Qiu, Y.; Parker, D.; Barnes, NC.; Johnson, M.; Pavord, L., et al. The effect of salmeterol/fluticasone propionate (SFC) on eosinophils and mast cells in COPD [Abstract]; American Thoracic Society 2005 International Conference; San Diego, California. May 20-25; 2005. p. A43Poster F36

Qiu YS, Davis P, Zhu J, Peachey L, Barnes NC, Pavord I, et al. Anti-inflammatory effects of salmeterol/fluticasone propionate (SFC) on airway T-lymphocyte populations in COPD. European Respiratory Journal. 2005; 26(Suppl 49):203s.

Qiu YS, Davis P, Zhu J, Peachey L, Barnes NC, Pavord I, et al. Effects of salmeterol/fluticasone propionate (SFC) on airway inflammation in COPD: a placebo-controlled study of endobronchial biopsies. European Respiratory Journal. 2005; 26(Suppl 49):203s.

SCO30005. A 13-week, double-blind, parallel-group, multicentre study to compare the bronchial antiinflammatory activity of the combination of salmeterol/fluticasone propionate (SERETIDETM/ ADVAIR ${ }^{\mathrm{TM}} / \mathrm{VIANI}^{\mathrm{TM}}$ ) $50 / 500 \mathrm{mcg}$ twice daily compared with placebo twice daily in patients with Chronic Obstructive Pulmonary Disease. GlaxoSmithKline Clinical Trials Register; 2005. http:ctr.gsk.co.uk

Zhu, J.; Qiu, Y.; Barnes, NC.; Johnson, M.; Pavord, I.; Jeffery, PK. The effect of salmeterol/ fluticasone propionate (SFC) on pro-inflammatory gene expression in COPD [Abstract]; American Thoracic Society 2005 International Conference; San Diego, California. May 20-25; 2005. p. A43Poster F6

Calverley 2003 \{published and unpublished data \} . Borgstrom, L.; Asking, L.; Olsson, H.; Peterson, S. Lack of interaction between disease severity and therapeutic response with budesonide/formoterol in a single inhaler [Abstract]; American Thoracic Society 100th International Conference; May 21-26; 2004. p. C22Poster 505

*Calverley PM, Bonsawat W, Cseke Z, Zhong N, Peterson S, Olsson H. Maintenance therapy with budesonide and formoterol in chronic obstructive pulmonary disease. European Respiratory Journal. 2003; 22(6):912-9. [PubMed: 14680078]

Calverley PMA, Cseke Z, Peterson S. Budesonide/formoterol reduces the use of oral corticosteroids in the treatment of COPD [Abstract]. European Respiratory Journal. 2003; 22(Suppl 45):P436.

Calverley PMA, Kuna P, Olsson H. COPD exacerbations are reduced by budesonide/formoterol in a single inhaler [Abstract]. European Respiratory Journal. 2003; 22(Suppl 45):P1587.

Calverley, PMA.; Olsson, H.; Symbicort, International; COPD Study Group. Budesonide/formoterol ina single inhaler sustains improvements in lung function over 12 months compared with 
monocomponents and placebo in patients with COPD [abstract]; American Thoracic Society 99th International Conference; 2003; p. B024Poster 418

Calverley, PMA.; Peterson, S. Combining budesonide/formoterol in a single inhale reduces exacerbation frequency in COPD [abstract]; American Thoracic Society 99th International Conference; 2003; p. D092Poster 211

Calverley, PMA.; Stahl, E.; Jones, PW. Budesonide/formoterol improves the general health status of patients with COPD [Abstract]; American Thoracic Society 2005 International Conference; San Diego, California. May 20-25; 2005. p. B93Poster 303

Calverley PMA, Szafranski W, Andersson A. Budesonide/formoterol is a well-tolerated long term maintenance therapy for COPD. European Respiratory Journal. 2005; 26(Suppl 49) Poster 1917.

Calverley PMA, Thompson NC, Olsson H. Budesonide/formoterol in a single inhaler sustains lung function improvements in COPD [Abstract]. European Respiratory Journal. 2003; 22(Suppl 45):P435.

Halpin, D.; Stahl, E.; Lundback, B.; Anderson, F.; Peterson, S. Treatment costs and number needed to treat (NNT) with budesonide/formoterol to avoid one exacerbation of COPD [Abstract]; American Thoracic Society 100th International Conference; May 21-26; 2004. p. D22Poster 525

Halpin, DMG.; Larsson, T.; Calverley, PMA. How many patients with COPD must be treated with budesonide/formoterol compared with formoterol alone to avoid 1 day of oral steroid use? [Abstract]; American Thoracic Society 2005 International Conference; San Diego, California. May 20-25; 2005. p. B93Poster 314

Jones, PW.; Stahl, E. Budesonide/formoterol in a single inhaler improves health status in patients with COPD [abstract]; American Thoracic Society 99th International Conference; 2003; p. B024Poster 419

Jones PW, Ståhl E. Budesonide /formoterol sustains clinically relevant improvements in health status in COPD [Abstract]. European Respiratory Journal. 2005; 26(Suppl 49) Abstract 1352.

Jones, PW.; Ståhl, E. Reducing exacerbations leads to a better health-related quality of life in patients with COPD; 13th ERS Annual Congress; Vienna. 27th September, 2003; 2003. p. P1586

Lofdahl CG. Reducing the impact of COPD exacerbations: Clinical efficacy of budesonide/formoterol. European Respiratory Review. 2004; 13(88):14-21.

Lofdahl CG, Andreasson E, Svensson K, Ericsson A. Budesonide/formoterol in a single inhaler improves health status in patients with COPD without increasing healthcare costs [Abstract]. European Respiratory Journal. 2003; 22(Suppl 45):P433.

Lofdahl CG, Ericsson A, Svensson K, Andreasson E. Cost effectiveness of budesonide/formoterol in a single inhaler for COPD compared with each monocomponent used alone. Pharmacoeconomics. 2005; 23(4):365-75. [PubMed: 15853436]

Dal Negro 2003 \{published data only\} . Dal Negro, R.; Micheletto, C.; Trevsian, F.; Tognella, S. [A98] Salmeterol and fluticasone 50ug/250ug BiD versus salmeterol 50ug bid and versus placebo in the long term treatment of COPD; Proceedings of the 98th International American Thoracic Society Conference; 2002; http://www.abstracts-on-line.com/abstracts/ATS

*Dal Negro RW, Pomari C, Tognella S, Micheletto C. Salmeterol \& fluticasone 50 microg/250 microg bid in combination provides a better long-term control than salmeterol 50 microg bid alone and placebo in COPD patients already treated with theophylline. Pulmonary Pharmacology and Therapeutics. 2003; 16(4):241-6. [PubMed: 12850128]

Hanania 2003 \{published and unpublished data\} . *Hanania NA, Darken P, Horstman D, Reisner C, Lee B, Davis S, et al. The efficacy and safety of fluticasone propionate ( 250 micro g)/ salmeterol (50 micro g) combined in the diskus inhaler for the treatment of COPD. Chest. 2003; 124(3):834-43. [PubMed: 12970006]

Hanania NA, Ramsdell J, Payne K, Davis S, Horstman D, Lee B, et al. Improvements in airflow and dyspnea in COPD patients following 24 weeks treatment with salmeterol 50mcg and fluticasone propionate $250 \mathrm{mcg}$ alone or in combination via the diskus. American Journal of Respiratory \& Critical Care Medicine. 2001; 163(5 Suppl):A279.

Horstman D, Darken P, Davis S, Lee B. Improvements in FEV1 and symptoms in poorly reversible COPD patients following treatment with salmeterol $50 \mathrm{mcg} /$ fluticasone propionate $250 \mathrm{mcg}$ combination [Abstract]. European Respiratory Journal. 2003; 22(Suppl 45):P434. 
Mahler, DA.; Darken, P.; Brown, CP.; Knobil, K. Predicting lung function responses to combination therapy in chronic obstructive pulmonary disease (COPD) [Abstract]; National COPD Conference; Arlington, Virginia. 2003; Abstract 1081

Mahler DA, Darken P, Brown CP, Knobil K. Predicting lung function responses to salmeterol/ fluticasone propionate combination therapy in COPD [Abstract]. European Respiratory Journal. 2003; 22(Suppl 45):P429.

SFCA3007. A randomized, double-blind, placebo-controlled, parallel-group trial evaluating the safety and efficacy of the DISKUS formulations of Salmeterol (SAL) 50mcg BID and Fluticasone Propionate (FP) 250mcg BID individually and in combination as Salmeterol 50mcg/Fluticasone Propionate 250mcg BID (SFC 50/250) compared to placebo in COPD subjects. GlaxoSmithKline Clinical Trials Register; 2005. http:ctr.gsk.co.uk

Spencer M, Wire P, Lee B, Chang CN, Darken P, Horstman D. Patients with COPD using salmeterol/ fluticasone propionate combination therapy experience improved quality of life. European Respiratory Journal. 2003; 22(Suppl 45):51s.

Spencer MD, Karia N, Anderson J. The clinical significance of treatment benefits with the salmeterol/ fluticasone propionate 50/500mcg combination in COPD. European Respiratory Journal. 2004; 24(Suppl 48):290s.

Mahler 2002 \{published and unpublished data $\quad$. Mahler, DA.; Darken, P.; Brown, CP.; Knobil, K. Predicting Lung Function Responses to Combination Therapy in Chronic Obstructive Pulmonary Disease (COPD). 2003. http://www.abstracts2view.com

* Mahler DA, Wire P, Horstman D, Chang CN, Yates J, Fischer T, et al. [Effectiveness of fluticasone propionate and salmeterol combination delivered via the diskus device in the treatment of chronic obstructive pulmonary disease]. American Journal of Respiratory Critical Care Medicine. 2002; 166(8):1084-91. [PubMed: 12379552]

SFCA3006. A randomized, double-blind, placebo-controlled, parallel-group trial evaluating the safety and efficacy of the DISKUS formulations of Salmeterol (SAL) 50mcg BID and Fluticasone Propionate (FP) 500mcg BID individually and in combination as Salmeterol 50mcg/Fluticasone Propionate 500mcg BID (SFC 50/500) compared to placebo in COPD subjects. GlaxoSmithKline Clinical Trials Register; 2005. http:ctr.gsk.co.uk

Spencer M, Wire P, Lee B, Chang CN, Darken P, Horstman D. Patients with COPD using salmeterol/ fluticasone propionate combination therapy experience improved quality of life. European Respiratory Journal. 2003; 22(Suppl 45):51s.

Spencer, MD.; Anderson, JA. Salmeterol/fluticasone combination produces clinically important benefits in dyspnea and fatigue [Abstract]; American Thoracic Society 2005 International Conference; San Diego, California. May 20-25; 2005. p. B93Poster 308

Spencer MD, Karia N, Anderson J. The clinical significance of treatment benefits with the salmeterol/ fluticasone propionate 50/500mcg combination in COPD. European Respiratory Journal. 2004; 24(Suppl 48):290s.

O’Donnell 2006 \{published and unpublished data\} . Celli B, Emmett A, Crater G, Kalberg C. Salmeterol/fluticasone propionate (SFC) improves the inspiratory to total lung capacity ratio (IC/ TLC) and exercise endurance time in patients with COPD. European Respiratory Journal. 2006; 28(Suppl 50):764s.

Make B, Emmett A, Crater G, O’Dell D, Kalberg C. Improvement in exercise endurance time (EET) with fluticasone propionate/salmeterol correlations with spirometry and plethysmography. Proceedings of the American Thoracic Society. 2006:A212.

*O'Donnell DE, Sciurba F, Celli B, Mahler DA, Webb KA, Kalberg CJ, et al. Effect of fluticasone propionate/salmeterol on lung hyperlation and exercise endurance in COPD. Chest. 2006; 130(3): 647-56. [PubMed: 16963658]

SCO40030. A randomized, double-blind, placebo-controlled, parallel group clinical trial evaluating the effect of the fluticasone propionate/salmeterol combination product $250 / 50 \mathrm{mcg}$ bid via DISKUS and salmeterol 50mcg bid via DISKUS on lung hyperinflation in subjects with chronic obstructive pulmonary disease (COPD). GlaxoSmithKline Clinical Trials Register; 2005. http:ctr.gsk.co.uk

SCO100540 \{unpublished data only\} . SCO100540. A multi-centre, randomised, double-blind, parallel group study to investigate the efficacy and safety of the salmeterol/fluticasone propionate combination at a strength of $50 / 500 \mu \mathrm{g} \mathrm{BD}$, compared with placebo via Accuhaler ${ }^{\mathrm{TM}}$, added to 
usual chronic obstructive pulmonary disease (COPD) therapy, in subjects with COPD for 24 weeks. 2006. www.ctr.gsk.co.uk

SFCT01 \{unpublished data only\} . SFCT01. A multicentre, randomised, double-blind, parallel group, placebo-controlled study to compare the efficacy and safety of inhaled salmeterol/ fluticasone propionate combination product $25 / 250 \mu \mathrm{g}$ two puffs bd and fluticasone propionate $250 \mu \mathrm{g}$ two puffs bd alone, all administered via metered dose inhalers (MDI), in the treatment of subjects with chronic obstructive pulmonary disease (COPD) for 52 weeks. GlaxoSmithKline Clinical Trials Register; 2005. http:ctr.gsk.co.uk

Szafranski 2003 \{published and unpublished data . Anderson P. [Budesonide/formoterol in a single inhaler (Symbicort) provides early and sustained improvement in lung function in moderate to severe COPD [Abstract]]. Thorax. 2002; 57(Suppl III):iii43.

Borgstrom, L.; Asking, L.; Olsson, H.; Peterson, S. Lack of interaction between disease severity and therapeutic response with budesonide/formoterol in a single inhaler [Abstract]; American Thoracic Society 100th International Conference; May 21-26; 2004. p. C22Poster 505

Calverley P, Pauwels R, Lofdahl CG, Svensson K, Higenbottam T, et al. Relationship between respiratory symptoms and medical treatment in exacerbations of COPD. European Respiratory Journal. 2005; 26(3):406-13. [PubMed: 16135720]

Calverley, PMA. Effect of budesonide/formoterol on severe exacerbations and lung function in moderate to severe COPD; Thorax. BTS Winter meeting; 2002; p. S145

Calverley PMA, Szafranski W, Andersson A. Budesonide/formoterol is a well-tolerated long term maintenance therapy for COPD. European Respiratory Journal. 2005; 26(Suppl 49) Poster 1917.

Calverley PMA, Thompson NC, Olsson H. Budesonide/formoterol in a single inhaler sustains lung function improvements in COPD [Abstract]. European Respiratory Journal. 2003; 22(Suppl 45):P435.

Campbell, LM.; Szafranski, W. Budesonide/formoterol in a single inhaler (Symbicort) provides sustained relief from symptoms in moderate to severe COPD; Thorax. BTS Winter meeting; 2002; p. S143

Campell, LW.; Szafranski, W. Budesonide/Formoterol in a single inhaler (Symbicort) reduces severe exacerbations in patients with moderate-severe COPD; Thorax. BTS Winter meeting; 2002; p. S141

Dahl R, Cukier A, Olsson H. Budesonide/formoterol in a single inhaler reduces severe and mild exacerbations in patients with moderate to severe COPD. European Respiratory Journal. 2002; 20(Suppl 38):242. P1575. [PubMed: 12166575]

Egede F, Menga G. Budesonide/formoterol in a single inhaler provides sustained relief from symptoms and night-time awakenings in moderate-severe COPD: results from symptoms and night-time awakenings in moderate to severe COPD: results from a 1-year study. European Respiratory Journal. 2002; 20(Suppl 38):242. P1574. [PubMed: 12166575]

Halpin, D.; Stahl, E.; Lundback, B.; Anderson, F.; Peterson, S. Treatment costs and number needed to treat (NNT) with budesonide/formoterol to avoid one exacerbation of COPD [Abstract]; American Thoracic Society 100th International Conference; May 21-26; 2004. p. D22Poster 525

Jones PW, Stahl E, Svensson K. Improvement in health status in patients with moderate to severe COPD after treatment with budesonide/formoterol in a single inhaler. European Respiratory Journal. 2002; 20(Suppl 38):250. P1613.

Korsgaard J, Sansores R. Budesonide/formoterol (single inhaler) provides sustained relief from shortness of breath and chest tightness in a 1-year study of patients with moderate to severe COPD. European Respiratory Journal. 2002; 20(Suppl 38):242. P1577. [PubMed: 12166575]

Lange P, Saenz C. Budesonide/formoterol in a single inhaler is well tolerated in patients with moderate to severe COPD: results of a 1 year study. European Respiratory Journal. 2002; 20(Suppl 38):242. P1573. [PubMed: 12166575]

Lofdahl CG. Reducing the impact of COPD exacerbations: Clinical efficacy of budesonide/formoterol. European Respiratory Review. 2004; 13(88):14-21.

Milanowski J, Nahabedian S. Budesonide/formoterol in a single inhaler acts rapidly to improve lung function and relieve symptoms in patients with moderate to severe COPD. European Respiratory Journal. 2002; 20(Suppl 38):242. P1576. [PubMed: 12166575] 
*Szafranski W, Cukier A, Ramirez A, Menga G, Sansores R, Nahabedian S, et al. Efficacy and safety of budesonide/formoterol in the management of chronic obstructive pulmonary disease. European Respiratory Journal. 2003; 21(1):74-81. [PubMed: 12570112]

TORCH \{published and unpublished data\} . Calverley P, Celli B, Anderson JA, Ferguson GT, Jenkins C, Jones PW, et al. The TOwards a Revolution in COPD Health (TORCH) study: salmeterol/fluticasone propionate improves survival in COPD over three years. Respirology. 2006; 11(Suppll 5):A149.

Calverley PM, Celli B, Anderson JA, Ferguson GT, Jenkins C, Jones PW, et al. The towards a revolution in COPD health (TORCH) study: fluticasone propionate /salmeterol improves survival in COPD over three years. Chest. 2006; 130(4):122s.

*Calverley PMA, Anderson JA, Celli B, Ferguson GT, Jenkins C, Jones PW, et al. Salmeterol and fluticasone propionate and survival in chronic obstructive pulmonary disease. New England Journal of Medicine. 2007; 356(8):775-89. [PubMed: 17314337]

Calverley PMA, Celli B, Andersen JA, Ferguson GT, Jenkins C, Jones PW, et al. The TORCH (towards a revolution in COPD health) study salmeterol/fluticasone propionate (SFC) improves survival in COPD over three years. European Respiratory Journal. 2006; 28(Suppl 50):34s.

Calverley PMA, Celli B, Ferguson G, Jenkins C, Jones PW, Pride NB, et al. Baseline characteristics of the first 5,000 COPD patients enrolled in the TORCH survival study. European Respiratory Journal. 2003; 22(Suppl 45):578s.

Celli B, Calverley PM, Anderson JA, Ferguson GT, Jenkins C, Jones P, et al. The TOwards a Revolution in COPD HEalth (TORCH) Study: salmeterol/fluticasone propionate reduces the rate of exacerbations over three years. Respirology. 2006; 11(Suppl 5):A140.

Celli B, Calverley PMA, Anderson JA, Ferguson GT, Jenkins C, Jones PW, et al. The TORCH (TOwards a Revolution in COPD Health) study: salmeterol/fluticasone propionate (SFC) improves health status, reduces exacerbations and improves lung function over three years. European Respiratory Journal. 2006; 28(Suppl 50):34s.

Corhay JL, Louis R. Clinical study of the month: the TORCH study (TOwards a Revolution in COPD Health) [L'étude clinique du mois. L'étude TORCH (TOwards a Revolution in COPD Health): vers une revolution de la santé des patients souffrant de BPCO]. Revue Medicale de Liege. 2007; 62(4):230-4. [PubMed: 17566394]

Ferguson GT, Calverley PM, Anderson JA, Celli B, Jenkins C, Jones PW, et al. The towards a revolution in COPD health (TORCH) study: fluticasone propionate/salmeterol is well tolerated in patients with COPD over 3 years. Chest. 2006; 130(4):178s.

Ferguson GT, Calverley PMA, Anderson JA, et al. The TORCH (TOwards a Revolution in COPD Health) study: salmeterol/fluticasone propionate (SFC) improves survival in COPD over three years. European Respiratory Journal. 2006; 28(Suppl 50):34s.

Jenkins, CR.; Calverley, PMA.; Celli, B.; Ferguson, G.; Jones, PW.; Pride, N., et al. Seasonal Patterns of Exacerbation Rates in the TORCH Survival Study. 2007. p. A839http://

www.abstracts2view.com

Jones, PW.; Calverley, P.; Celli, B.; Ferguson, G.; Jenkins, C.; Pride, N. Trans-Regional Validity of the SGRQ in the TORCH Survival Study. 2007. p. A122http://www.abstracts2view.com

Jones PW, Calverley PM, Celli B, Anderson JA, Ferguson GT, Jenkins C, et al. The towards study a revolution in COPD health (TORCH) study: fluticasone propionate/salmeterol improves and sustains health status in COPD over 3 years. Chest. 2006; 130(4):177s.

McGarvey LP, John M, Anderson JA, Zvarich MT, Wise RA. Ascertainment of cause-specific mortality in COPD: operations of the TORCH Clinical Endpoint Committee. Thorax. 2007; 62:411-5. [PubMed: 17311843]

SCO30003. A multicentre, randomised, double-blind, parallel group, placebo-controlled study to investigate the long-term effects of salmeterol/fluticasone propionate (SERETIDE®/VIANI®/ ADVAIR $®) ~ 50 / 500 \mathrm{mcg}$ bd, salmeterol $50 \mathrm{mcg}$ bd and fluticasone propionate $500 \mathrm{mcg}$ bd, all delivered via the DISKUS®/ACCUHALER $®$ inhaler, on the survival of subjects with chronic obstructive pulmonary disease (COPD) over 3 years of treatment. 2006. www.ctr.gsk.co.uk 
Vestbo J, Calverley P, Celli B, Ferguson G, Jenkins C, Jones P, et al. The TORCH (TOwards a Revolution in COPD Health) survival study protocol. European Respiratory Journal. 2004; 24(2): 206-10. [PubMed: 15332386]

Wise, RA.; McGarvey, LP.; John, M.; Anderson, JA.; Zvarich, MT. Reliability of cause-specific mortality adjudication in a COPD clinical trial. 2007. p. A120http://www.abstracts2view.com

TRISTAN \{published and unpublished data\} . *Calverley P, Pauwels R, Vestbo J, Jones P, Pride $\mathrm{N}$, Gulsvik A, et al. Combined salmeterol and fluticasone in the treatment of chronic obstructive pulmonary disease: a randomised controlled trial. Lancet. 2003; 361(9356):449-56. [PubMed: 12583942]

Calverley, PMA.; Pauwels, RA.; Vestbo, J.; Jones, PW.; Pride, NB.; Gulsvik, A., et al. Clinical improvements with salmeterol / fluticasone propionate combination in differing severities of COPD. 2003. p. A035http://www.abstracts2view.comPoster D50

Calverley, PMA.; Pauwels, RA.; Vestbo, J.; Jones, PW.; Pride, NB.; Gulsvik, A., et al. Salmeterol/ Fluticasone propionate combination for one year provides greater clinical benefit than its individual components; Proceedings of the 98th International American Thoracic Society Conference; 2002; p. A98http://www.abstracts-on-line.com/abstracts/ATSPoster 306

Calverly PMA, Pauwels R, Vestbo J, Jones P, Pride N, Gulsvik A, et al. Safety of salmeterol/ fluticasone propionate combination in the treatment of chronic obstructive pulmonary disease. European Respiratory Journal. 2002; 20(Suppl 38):242. P1572. [PubMed: 12166575]

Hunjan, MK.; Chandler, F. Numbers needed to treat (NNT) to avoid an exacerbation in patients with chronic obstructive pulmonary disease (COPD) using salmeterol/fluticasone propionate combination (SFC) and associated costs [Abstract]; American Thoracic Society 100th International Conference; May 21-26; 2004. p. D22Poster 503

Hunjan MK, Williams DT. Costs of avoiding exacerbations in patients with chronic obstructive pulmonary disease (COPD) treated with salmeterol/fluticasone propionate combination (seretide) and salmeterol. European Respiratory Journal. 2004; 24(Suppl 48):291s.

Hunjan MK, Williams DT. Salmeterol/fluticasone propionate combination is clinically effective in avoiding exacerbations in patients with moderate/severe COPD. European Respiratory Journal. 2004; 24(Suppl 48):513s.

Jones, PW.; Edin, HM.; Anderson, J. Salmeterol/fluticasone propionate combination improves health status in COPD patients; Proceedings of the 98th International American Thoracic Society Conference; 2002; p. A39http://www.abstracts-online.com/abstracts/ATSPoster K39

Jones PW, Ståhl E. Budesonide /formoterol sustains clinically relevant improvements in health status in COPD [Abstract]. European Respiratory Journal. 2005; 26(Suppl 49) Abstract 1352.

Jones, PW.; Vestbo, J.; Pauwels, RA.; Calverley, PMA.; Anderson, JA.; Spencer, MD. Informative drop out in COPD studies. Investigation of health status of withdrawals in the TRISTAN study; 13th ERS Annual Congress; Vienna. 27 Spetember 2003; 2003. p. P1593

Nitschmann S. Inhalational combination therapy in chronic obstructive lung disease. Tristan study. German Internist. 2004; 45(6):727-8.

Pauwels, R.; Vestbo, J.; Calverley, PMA.; Jones, PW.; Pride, NB.; Gulsvik, A. Characterization of exacerbations in the TRISTAN study of salmeterol / flucticasone propionate (SFC) combination in moderate to severe COPD. 2003. http://www.abstracts2view.com

Pauwels RA, Calverly PMA, Vestbo J, Jones PW, Pride N, Gulsvik A, et al. Reduction of exacerbations with salmeterol/flutciasone combination $50 / 500 \mathrm{mcg}$ bd in the treatment of chronic obstructive pulmonary disease. European Respiratory Journal. 2002; 20(Suppl 38):240. P1569.

SFCB3024. A multicentre, randomised, double-blind, parallel group, placebo-controlled study to compare the efficacy and safety of the salmeterol/FP combination product at a strength of $50 / 500 \mathrm{mcg}$ bd with salmeterol $50 \mathrm{mcg}$ bd alone and FP $500 \mathrm{mcg}$ bd alone, delivered via the DISKUSTM/ACCUHALER ${ }^{\mathrm{TM}}$, in the treatment of subjects with chronic obstructive pulmonary disease (COPD) for 12 months. GlaxoSmithKline Clinical Trials Register; 2005. http:ctr.gsk.co.uk

Spencer M, Briggs AH, Grossman RF, Rance L. Development of an economic model to assess the cost effectiveness of treatment interventions for chronic obstructive pulmonary disease. Pharmacoeconomics. 2005; 23(6):619-37. [PubMed: 15960557] 
Spencer MD, Karia N, Anderson J. The clinical significance of treatment benefits with the salmeterol/ fluticasone propionate 50/500mcg combination in COPD. European Respiratory Journal. 2004; 24(Suppl 48):290s.

Vestbo J, Calverley PMA, Pauwels R, Jones P, Pride N, Gulsvik A, et al. Absence of gender susceptibility to the combination of salmeterol and fluticasone in the treatment of chronic obstructive pulmonary disease. European Respiratory Journal. 2002; 20(Suppl 38):240. P1570.

Vestbo J, Pauwels R, Anderson JA, Jones P, Calverley P. Early onset of effect of salmeterol and fluticasone propionate in chronic obstructive pulmonary disease. Thorax. 2005; 60(4):301-4. [PubMed: 15790985]

Vestbo, J.; Pauwels, RS.; Calverley, PMA.; Jones, PW.; Pride, NB.; Gulsvik, A. Salmeterol / fluticasone propionate combination produces improvement in lung function detectable within 24 Hours in moderate to severe COPD. 2003. http://www.abstracts2view.com

Vestbo J, Soriano JB, Anderson JA, Calverley P, Pauwels R, Jones P. Gender does not influence the response to the combination of salmeterol and fluticasone propionate in COPD. Respiratory Medicine. 2004; 98(11):1045-50. [PubMed: 15526804]

\section{References to studies excluded from this review}

Aaron 2004 \{published data only . Aaron SD, Vandemheen K, Ferguson D, FitzGerald M, Maltais F, Boureau J, et al. The Canadian optimal therapy of COPD trial: Design, organization and patient recruitment. Canadian Respiratory Journal. 2004; 11(8):581-5. [PubMed: 15611808]

Borgstrom 2003 \{published data only\} . *Borgstrom L, Lipniunas P. Budesonide/formoterol in Turbuhaler@ is not affected by storage in hot and humid conditions. A clinical pharmacokinetic comparison with fluticasone in Diskus ${ }^{\mathrm{TM}}$ [Abstract]. European Respiratory Journal. 2003; 22(Suppl 45):P1580.

Cazzola 2000 \{published data only . Cazzola M, Di Lorenzo G, Di Perna F, Calderaro F, Testi R, Centanni S. Additive effects of salmeterol and fluticasone or theophylline in COPD. Chest. 2000; 118(6):1576-81. [PubMed: 11115442]

Cazzola 2002a \{unpublished data only\} . Cazzola M, Santus P, Di Marco F, Boveri B, Castagna F, Carlucci P. Bronchodilator effect of an inhaled combination therapy with salmeterol + fluticasone and formoterol + budesonide in patients with COPD. European Respiratory Journal. 2002; 20(Suppl 38):386. P2396.

Cazzola 2003 \{published data only\}. Cazzola M, Salzillo A, De Giglio C, Noschee P, D'Amato G. Formoterol/budesonide (FBC) in the treatment of acute exacerbation of COPD [Abstract]. European Respiratory Journal. 2003; 22(Suppl 45):P1859.

Cazzola 2004 \{published data only\} . *Cazzola M, Noschese P, Centanni S, Santus P, Di Marco F, Spicuzza L, et al. Salmeterol/fluticasone propionate in a single inhaler device versus theophylline + fluticasone propionate in patients with COPD. Pulmonary Pharmacology \& Therapeutics. 2004; 17(3):141-5. [PubMed: 15123223]

Cazzola M, Noschese P, D'Amato G, Santus P, Centanni S. Salmeterol/fluticasone propionate in a single inhaler device (SLM/FP) versus theophyline (THEO) + FP in patients with COPD. European Respiratory Journal. 2002; 20(Suppl 38):386. P2393.

Cazzola 2004b \{published data only\} . Cazzola, M.; Santus, P.; Di Marco, F.; Carlucci, P.; Belloni, E.; Mondoni, M., et al. Onset of action of budesonide/formoterol in single inhaler vs. formoterol in patients with COPD [abstract]. American Thoracic Society 99th International Conference; 2003; p. B024Poster 410

*Cazzola M, Santus P, Di Marco F, Carlucci P, Mondoni M, Matera MG, et al. Onset of action of formoterol/budesonide in single inhaler vs. formoterol in patients with COPD. Pulmonary Pharmacology \& Therapeutics. 2004; 17(3):121-5. [PubMed: 15123220]

Chapman 2002 \{published data only\} . Chapman KR. Seretide for obstructive lung disease. Expert Opinion on Pharmacotherapy. 2002; 3(3):341-50. [PubMed: 11866683]

Donohue 2004 \{published data only\} .*Donohue JF, Kalberg C, Emmett A, Merchant K, Knobil K. A short-term comparison of fluticasone propionate/salmeterol with ipratropium bromide/ 
albuterol for the treatment of COPD. Treatments in Respiratory Medicine. 2004; 3(3):173-81. [PubMed: 15219176]

Donohue JF, Kalberg C, Emmett A, Merchant K, Knobil K. Fluticasone/salmeterol combination (250/50) provides greater improvement in lung function and symptoms than the combination of ipratropium/albuterol in patients with COPD. Chest. 2003; 124(4):128S.

Kalberg, C.; Emmett, A.; Bourne, E.; Merchant, K.; Knobil, K. Fluticasone propionate/salmeterol provides greater relief of dyspnea than ipratropium/albuterol in patients with COPD [Abstract]; American Thoracic Society 100th International Conference; May 21-26; 2004. p. C22Poster 528

Knobil K, Kalberg C, Merchant K, Emmett A, Cicale M. Maintenance of bronchodilator response for advair diskus 250/50 (fluticasone propionate/salmeterol) but not ipratropium/albuterol in patients with COPD [Abstract]. Chest. 2004; 126(4):807S.

Knobil K, Merchant K, Kalberg C, Emmett A, Cicale M. A comparison of patient perceived improvement in symptoms after initiating therapy with either advair diskus (fluticasone propionate/salmeterol) 250/50 or ipratropium/albuterol [Abstract]. Chest. 2004; 126(4 Suppl): 806S-b-807S-b.

Merchant, K.; Bourne, E.; Emmett, A.; Knobil, K.; Kalberg, C. Greater improvement in dyspnea with fluticasone propionate/salmeterol 250/50 compared with salmeterol or ipratropium in patients with COPD [Abstract]; American Thoracic Society 100th International Conference; May 21-26; 2004. p. C22Poster 526

Sciurba FC, Kalberg C, Emmett A, Merchant K, Brown C, Knobil K. Efficacy of advair diskus 250/50 (fluticasone propionate/salmeterol) or ipratropium/albuterol in patients with COPD associated with chronic bronchitis and/or emphysema [Abstract]. Chest. 2004; 126(4 Suppl):807S-a-808Sa.

Kardos 2007 \{published and unpublished data\} . Kardos P, Wencker M. Combination therapy with salmeterol and fluticasone propionate (SFC) is more effective than salmeterol (SAL) alone in reducing exacerbations of COPD [Abstract]. European Respiratory Journal. 2005; 26(Suppl 49) Abstract 1944.

*Kardos P, Wencker M, Glaab T, Vogelmeier C. Impact of salmeterol/fluticasone propionate versus salmeterol on exacerbations in severe chronic obstructive pulmonary disease. American Journal of Respiratory \& Critical Care Medicine. 2007; 175(2):144-9. [PubMed: 17053207]

SCO30006. A randomised, double-blind, parallel-group study to investigate the protective effect of the combination of fluticasone and salmeterol $(500 / 50 \mu \mathrm{g}$ bid via the DISKUS) compared with salmeterol (50 $\mu \mathrm{g}$ bid via the DISKUS) on the incidence of moderate to severe exacerbations in patients with severe chronic obstructive pulmonary disease (COPD) (GOLD III/IV). GlaxoSmithKline Clinical Trials Register; 2005. http:ctr.gsk.co.uk

Vogelmeier, C. Combination therapy with with salmeterol and fluticasone propionate (SFC) improves quality of life (QoL) more than salmeterol (SAL) alone in COPD; 42nd Nordic Lung Conference Trondheim; 2005; p. 13

Noschese 2003 \{published data only\} . Noschese P, De Michele F, D’Amato G, Cazzola M. Comparison of the acute effects of formoterol (F) and formoterol/budesonide combination (FBC) on blood gas tensions in patients with acute exacerbation of COPD (AECOPD)[Abstract]. European Respiratory Journal. 2003; 22(Suppl 45):P156.

SAM40116 \{unpublished data only . *SAM40116. A 12-week multi-centre, randomised, double blind, parallel group study to compare the efficacy of salmeterol/fluticasone propionate (Seretide TM) 50/100 mcg or 50/250 mcg twice daily and fluticasone propionate (Flixotide TM) $250 \mathrm{mcg}$ or $500 \mathrm{mcg}$ twice daily via the Diskus TM in subjects with asthma/COPD symptoms. GlaxoSmithKline Clinical Trials Register; 2005. http:ctr.gsk.co.uk

SCO100470 \{unpublished data only\}. SCO100470. A multicentre, randomised, double-blind, parallel group, 24-week study to compare the effect of the salmeterol/fluticasone propionate combination product $50 / 250 \mathrm{mcg}$, with salmeterol $50 \mathrm{mcg}$ both delivered twice daily via the DISKUS/ACCUHALER inhaler on lung function and dyspnoea in subjects with Chronic Obstructive Pulmonary Disease (COPD). GlaxoSmithKline Clinical Trials Register; 2006. http:ctr.gsk.co.uk

SCO40034 \{unpublished data only\} . A multicentre, randomised, double-blind, double dummy, parallel group 12-week exploratory study to compare the effect of the salmeterol/fluticasone 
propionate combination product (SERETIDE) 50/500mcg bd via the DISKUS/ACCUHALER inhaler with tiotropium bromide $18 \mathrm{mcg}$ od via the Handihaler inhalation device on efficacy and safety in patients with chronic obstructive pulmonary disease (COPD. GlaxoSmithKline Clinical Trials Register; 2005. http:ctr.gsk.co.uk

Soriano 2002 \{published data only\} . *Soriano JB, Vestbo J, Pride NB, Kiri V, Maden C, Maier WC. Survival in COPD patients after regular use of fluticasone propionate and salmeterol in general practice. European Respiratory Journal. 2002; 20(4):797-8. [PubMed: 12412666]

Sun 2004 \{published data only\} . Sun, D.; Yang, J.; Xu, X. Combined seretide and zafirlukast in the treatment of chronic obstructive pulmonary disease (COPD) [Abstract]. American Thoracic Society 100th International Conference; May 21-26. 2004; p. C22Poster 524

Vestbo 2004 \{published data only\} . Vestbo J. Combined salmeterol and fluticasone in the treatment of chronic obstructive pulmonary disease. Dan. Ugeskrift for Laeger. 2004; 166(4): 271-4.

Wouters 2005 \{published data only\} . Creutzberg, EC.; Bookelmann, YJH.; Hop, WCJ.; Postma, DS.; Wouters, EFM. Determinants of health status in patients with COPD. 2003. p. A107http:// www.abstracts2view.comPoster C31

Creutzberg, EC.; Bookelmann, YJH.; Hop, WCJ.; Postma, DS.; Wouters, EFM. Predicting factors for an acute exacerbation of COPD in the COSMIC study. 2003. http://www.abstracts2view.com

Rizzato G. COPD: Immediate and sustained deterioration after withdrawal of fluticasone in patients under therapy with salmeterol + fluticasone. Internista. 2005; 13(4):225-30.

Wouters EF, Postma DS, Fokkens B, Hop WC, Prins J, Kuipers AF, The Cosmic COPD and Serentide: A Multi-Center Intervention and Characterization Study Group. Withdrawal of fluticasone propionate from combined salmeterol/fluticasone treatment in patients with COPD causes immediate and sustained disease deterioration: a randomised controlled trial [A remocao do propionato de fluticasona da terapeutica combinada com salmeterol/fluticasona em doentes com DPOC causa agravamento imediato e mantido; um estudo randomizado e controlado]. Revista Portuguesa De Pneumologia. 2005; 11(6):587-9. [PubMed: 16514718]

*Wouters EF, Postma DS, Fokkens B, Hop WC, Prins J, Kuipers AF, et al. Withdrawal of fluticasone propionate from combined salmeterol/fluticasone treatment in patients with COPD causes immediate and sustained disease deterioration: a randomised controlled trial. Thorax. 2005; 60(6):480-7. [PubMed: 15923248]

Wouters EFM. COPD and seretide: a multi-center intervention and characterisation (COSMIC) study: a rationale and baseline characteristics. European Respiratory Journal. 2002; 20(38):P1571.

Wouters, EFM.; Postma, DS.; Fokkens, B.; Hop, WCJ.; Creutzberg, EC. One year withdrawal of fluticasone after three month treatment with combined fluticasone/salmeterol in COPD [Abstract]. American Thoracic Society 100th International Conference; May 21-26. 2004; p. C22Poster 516

\section{References to studies awaiting assessment}

Aaron 2007 \{published data only . Aaron SD, Vandemheen KL, Fergusson D, Maltais F, Bourbeau J, Goldstein R, et al. Tiotropium in combination with placebo, salmeterol, or fluticasone-salmeterol for treatment of chronic obstructive pulmonary disease: a randomized trial. Annals of Internal Medicine. 2007; 146(8):545-55. [PubMed: 17310045]

Kaplan A. Effects of tiotropium combined with either salmeterol or salmeterol/fluticasone in moderate to severe COPD. Primary Care Respiratory Journal. 2007; 16(4):258-60.

Bourbeau 2007 \{published data only\} . Bourbeau J, Christodoulopoulos P, Maltais F, Yamauchi Y, Olivenstein R, Hamid Q. Effect of salmeterol/fluticasone propionate on airway inflammation in COPD: A randomised controlled trial. Thorax. 2007; 62(11):938-43. [PubMed: 17557771]

Cukier 2007 \{published data only\} . Cukier A, Ferreira CAS, Stelmach R, Ribeiro M, Cortopassi F, Calverley PMA. The effect of bronchodilators and oxygen alone and in combination on selfpaced exercise performance in stable COPD. Respiratory Medicine. 2007; 101(4):743-53. 
Golabi 2006 \{published data only\} . Golabi P, Topaloglu N, Karakurt S, Celikel T. Effects of tiotropium and salmeterol/fluticasone combination on lung hyperinflation dyspnea and exercise tolerance in COPD [Abstract]. European Respiratory Journal. 2006; 28(Suppl 50):33s.

Haque 2006 \{published data only\} . Haque RA, Torrego A, Essilfie-Quaye S, Kharitonov SA, Johnson M, Adcock IM, et al. Effect of salmeterol and fluticasone on glucocorticoid receptor translocation in sputum macrophages and peripheral blood mononuclear cells from patients with chronic obstructive pulmonary disease. Proceedings of the American Thoracic Society. 2006:A848.

INSPIRE \{published data only\} . GlaxoSmithKline (SCO40036). [accessed 8th April 2008] Multicentre, randomised, double-blind, double-dummy, parallel group, 104-week study to compare the effect of the salmeterol/fluticasone propionate combination product (SERETIDE*) $50 / 500 \mathrm{mcg}$ delivered twice daily via the DISKUS*/ACCUHALER* inhaler with tiotropium bromide $18 \mathrm{mcg}$ delivered once daily via the HandiHaler inhalation device on the rate of health care utilisation exacerbations in subjects with severe chronic obstructive pulmonary disease (COPD). http://ctr.gsk.co.uk

Seemungal T, Stockley R, Calverley P, Hagan G, Wedzicha JA. Investigating new standards for prophylaxis in reduction of exacerbations - The INSPIRE study methodology. Journal of Chronic Obstructive Pulmonary Disease. 2007; 4(3):177-83. [PubMed: 17729060]

Wedzicha J, Stockley R, Seemungal T, Hagan G, Calverley P. The INSPIRE study: effect of salmeterol/fluticasone propionate versus tiotropium bromide on COPD exacerbations. Respirology. 2007; 12(Suppl 4):A112.

Lindberg 2007 \{published data only\} . *Lindberg A, Szalai Z, Pullerits T, Radeczky E. Fast onset of effect of budesonide/formoterol versus salmeterol/fluticasone and salbutamol in patients with chronic obstructive pulmonary disease and reversible airway obstruction. Respirology. 2007; 12(5):732-9. [PubMed: 17875063]

Lindberg A, Szalai Z, Pullertis T, Radeczky El. Budesonide/formoterol (B/F) has an onset of action that is similar to salbutamol and faster than salmeterol/fluticasone in patients with COPD. European Respiratory Journal. 2006; 28(Suppl 50):214s.

Schermer 2007 \{published data only\} . Schermer TR, Albers JM, Verblackt HW, Costongs RJ, Westers P. Lower inhaled steroid requirement with a fluticasone/salmeterol combination in family practice patients with asthma or COPD. Family Practice. 2007; 24(2):181-8. [PubMed: 17251178]

SCO100250 \{unpublished data only\} . GlaxoSmithKline (SCO100250). [accessed 8th April 2008] A randomized, double-blind, parallel-group, 52-week study to compare the effect of fluticasone propionate/salmeterol DISKUS 250/50mcg bid with salmeterol DISKUS 50mcg bid on the annual rate of moderate/severe exacerbations in subjects with chronic obstructive pulmonary disease (COPD). http://ctr.gsk.co.uk

SCO40043 \{unpublished data only . SCO40043. [accessed 8th April 2008] A randomized, double-blind, parallel-group, 52-week study to compare the effect of fluticasone propionate/ salmeterol DISKUS® $250 / 50 \mathrm{mcg}$ bid with salmeterol DISKUS® $50 \mathrm{mcg}$ bid on the annual rate of moderate/severe exacerbations in subjects with chronic obstructive pulmonary disease (COPD). http://ctr.gsk.co.uk

Sethi 2006 \{published data only\} . Sethi S, Grove L, Wrona C, Maloney J. Prevalence of bacterial colonization in COPD is not altered by fluticasone/salmeterol. Proceedings of the American Thoracic Society. 2006:A115.

SHINE \{unpublished data only\} . Astrazeneca (D5899C00002). [Accessed 8th April, 2008] A 6Month double-blind, double-dummy, randomized, parallel group, multicenter efficacy \& safety study of Symbicort ${ }^{\circledR}$ pMDI $2 \times 160 / 4.5 \mathrm{mg} \& 80 / 4.5 \mathrm{mg}$ bid compared to Formoterol TBH, Budesonide pMDI ( $\&$ the combination) \& placebo in COPD patients. 2008. www.astrazenecaclinicaltrials.com

Sutherland 2006 \{published data only\} . Sutherland ER, Moss TA, Stevens AD, Pak J, Martin RJ. Modulation of sputum gene expression in COPD by fluticasone /salmeterol. European Respiratory Journal. 2006; 28(Suppl 50):662s. 
Trofimenko 2006 \{published data only\} . Trofimenko IN, Chernyak BA. The efficacy of salmeterol/fluticasone (SF) for 6 month's therapy at severe COPD patients. European Respiratory Journal. 2006; 28(Suppl 50):30s.

Zheng 2006 \{published data only\} . *Zheng J, Zhong N, Yang L, Wu Y, Chen P, Wen Z, et al. The efficacy and safety of fluticasone propionate $500 \mathrm{mg} / \mathrm{salmeterol} 50 \mathrm{mg}$ combined via diskus/ accuhaler in Chinese patients with chronic obstructive pulmonary disease (COPD). Chest. 2006; 130(4):182s.

Zhong N, Zheng J, Yang L, Wu Y, Chen P, Wen Z, et al. The efficacy and safety of salmeterol 50 $\mu$ / fluticasone propionate 500 $\mu$ combined via accuhaler in Chinese patients with chronic obstructive pulmonary disease [Abstract]. Respirology. 2006; 11(Suppl 5):A150.

\section{References to ongoing studies}

Morgan 2004 \{published data only\} .*Salmetrol xinafoate/fluticasone propionate combination product in the treatment of chronic obstructive pulmonary disease. $2001 \mathrm{http}: / /$ www.nrr.nhs.uk/. Dr Mike Morgan. Publication ID: N0123137844.

\section{Additional references}

Appleton 2006 . Appleton S, Poole P, Smith B, Veale A, Lasserson TJ, Chan MM, et al. Long-acting beta2-agonists for poorly reversible chronic obstructive pulmonary disease. Cochrane Database of Systematic Reviews. 2006; (3) Art. No.: CD001104. DOI: 10.1002/14651858.CD001104.pub2.

ATS 1995 . American Thoracic Society. Standards for the diagnosis and care of patients with chronic obstructive pulmonary disease. American Journal of Respiratory \& Critical Care Medicine. 1995; 152(5 Pt 2):S77-121. [PubMed: 7582322]

Boyd 1995 . Boyd G, Crawford C. Salmeterol for the treatment of patients with chronic obstructive pulmonary disease (COPD). European Respiratory Journal. 1995; 8(Suppl 19):167. [PubMed: 7744184]

Burge 2000 . Burge PS, Calverley PM, Jones PW, Spencer S, Anderson JA, Maslen TK. Randomised, double blind, placebo controlled study of fluticasone propionate in patients with moderate to severe chronic obstructive pulmonary disease: the ISOLDE trial y of fluticasone propionate in patients with moderate to severe chronic obstructive pulmonary disease: the ISOLDE trial. British Medical Journal. 2000; 320(7245):1297-1303. [PubMed: 10807619]

Burge 2003 . Burge PS, Calverley PMA, Jones PW, Spencer S, Anderson JA. Prednisolone response in patients with chronic obstructive pulmonary disease: results from the ISOLDE study. Thorax. 2003; 58(8):654-8. [PubMed: 12885977]

Calverley 2003a . Calverley PMA, Burge PS, Spencer S, Anderson JA, Jones PW. Bronchodilator reversibility testing in chronic obstructive pulmonary disease. Thorax. 2003; 58(8):659-64. [PubMed: 12885978]

Calverley 2005 . Calverley P, Pauwels R, Lofdahl CG, Svensson K, Higenbottam T, Carlsson LG, et al. Relationship between respiratory symptoms and medical treatment in exacerbations of COPD. European Respiratory Journal. 2005; 26(3):406-13. [PubMed: 16135720]

Campbell 2001 . Campbell D, Kelly K, Manser R. Combined corticosteroid and longacting bronchodilator in one inhaler for chronic asthma. Cochrane Database of Systematic Reviews. 2001; (4)

Dahl 2001 . Dahl R, Greefhorst LAPM, Nowak D, Nonikov V, et al. Inhaled formoterol dry powder versus ipratroprium bromide in chronic obstructive pulmonary disease. American Journal of Respiratory \& Critical Care Medicine. 2001; 164(5):778-84. [PubMed: 11549532]

Ernst 2007 . Ernst P, Gonzalez AV, Brassard P, Suissa S. Inhaled Corticosteroid Use in COPD and the Risk of Hospitalization for Pneumonia. American Journal of Respiratory and Critical Care Medicine. 2007 Epub ahead of print.

GOLD 2006 . Global Strategy for Diagnosis, Management, Prevention of COPD. Global Initiative for Chronic Obstructive Lung Disease (GOLD). 2006. http://www.goldcopd.org 
Ito 2001 . Ito K, Lim S, CaramorI G, Chung KF, Barnes PJ, Adcock IM. Cigarette smoking reduces histone deacetylase 2 expression, enhances cytokine expression, and inhibits glucocorticoid actions in alveolar macrophages. FASEB Journal. 2001; 15(6):1110-2. [PubMed: 11292684]

Jadad 1996 . Jadad AR, Moore RA, Carroll D, Jenkinson C, Reynolds DJ, Gavaghan DJ, et al. Assessing the quality of reports of randomized clinical trials: is blinding necessary? Controlled Clinical Trials. 1996; 17(1):1-12. [PubMed: 8721797]

Jones 1997 . Jones PW, Bosh TK. Quality of life changes in COPD patients treated with salmeterol. American Journal of Respiratory and Critical Care Medicine. 1997; 155(4):1283-9. [PubMed: 9105068]

Jones 2002 . Jones PW. Interpreting thresholds for a clinically significant change in health status in asthma and COPD. European Respiratory Journal. 2002; 19(3):398-404. [PubMed: 11936514]

Lung Health 2000 . The Lung Health Study Research Group. Effect of inhaled triamcinolone on the decline in pulmonary function in chronic obstructive pulmonary disease. New England Journal of Medicine. 2000; 343(26):1902-9. [PubMed: 11136260]

McGarvey 2007 . McGarvey LP, John M, Anderson JA, Zvarich MT, Wise RA. Ascertainment of Cause-Specific Mortality in COPD - Operations of the TORCH Clinical Endpoint Committee. Thorax. 2007; 62:411-5. [PubMed: 17311843]

Nannini 2007a . Nannini L, Cates CJ, Lasserson TJ, Poole P. Combined corticosteroid and longacting beta-agonist in one inhaler versus long-acting beta-agonists for chronic obstructive pulmonary disease. Cochrane Database of Systematic Reviews. 2007; (4) CD006829. Art. No.: CD006829. DOI: 10.1002/14651858.CD006829.

Nannini 2007b . Nannini L, Cates CJ, Lasserson TJ, Poole P. Combined corticosteroid and longacting beta-agonist in one inhaler versus inhaled steroids for chronic obstructive pulmonary disease. Cochrane Database of Systematic Reviews. 2007; (4) CD006826. Art. No.: CD006826. DOI: $10.1002 / 14651858 . C D 006826$.

NHLB/WHO 2001 . NHLBI/WHO. Global Strategy for the diagnosis, management, and prevention of chronic obstructive pulmonary disease. American Journal of Respiratory and Critical Care Medicine. 2001; 163:1256-76. [PubMed: 11316667]

Ni Chroinin 2004 . Ni Chroinin M, Greenstone IR, Ducharme FM. Addition of inhaled long-acting beta2-agonists to inhaled steroids as first line therapy for persistent asthma in steroid-naive adults. Cochrane Database of Systematic Reviews. 2004; (4) CD005307.

Ni Chroinin 2005 . Ni Chroinin M, Greenstone IR, Danish A, Magdolinos H, Masse V, Zhang X, Ducharme FM. Long-acting beta2-agonists versus placebo in addition to inhaled corticosteroids in children and adults with chronic asthma. Cochrane Database of Systematic Reviews. 2005; (4) Art. No.: CD005535. DOI: 10.1002/14651858.CD005535.

NICE 2004 . National Collaborating Centre for Chronic Conditions. Managament of chronic obstructive pulmonary disease in primary and secondary care. 2004. http://www.nice.org.uk

Pauwels 1999a . Pauwels RA, Lofdahl CG, Laitinen LA, Schouten JP, Postma DS, Pride NB, et al. Long-term treatment with inhaled budesonide in persons with mild chronic obstructive pulmonary disease who continue smoking. European Respiratory Society Study on Chronic Obstructive Pulmonary Disease. New England Journal of Medicine. 1999; 340(25):1948-53. [PubMed: 10379018]

Pauwels 1999b . Pauwels RA, Lofdahl C, Laitinen LA, Schouten JP, Postma DS, Pride NB, et al. Long-term treatment with inhaled budesonide in persons with mild chronic obstructive pulmonary disease who continue smoking. New England Journal of Medicine. 1999; 340(25): 1948-53. [PubMed: 10379018]

Pedersen 1996 . Pedersen B, Dahl R, Karlstrom R, Peterson CG, Venge P. Eosinophil and neutrophil activity in asthma in a one-year trial with inhaled budesonide. The impact of smoking. American Journal of Respiratory \& Critical Care Medicine. 1996; 153(5):1519-29. [PubMed: 8630596]

Roisin 2000 . Rodriguez-Roisin R. Toward a consensus definition of for COPD exacerbations. Chest. 2000; 117(5):398s-401s. [PubMed: 10843984]

Rossi 2002 . Rossi A, Kristufek P, Levine BE, Thomson MH, Till D, Kottakis J, et al. Comparison of the efficacy, tolerability, and safety of formoterol dry powder and oral, slow-release theophylline in the treatment of COPD. Chest. 2002; 121(4):1058-64. [PubMed: 11948033] 
Suissa 2006 . Suissa S. Statistical treatment of exacerbations in therapeutic trials of chronic obstructive pulmonary disease. American Journal of Respiratory \& Critical Care Medicine. 2006; 173(8):842-6. [PubMed: 16439716]

Sutherland 2003 . Sutherland ER, Allmers H, Ayas NT, Venn AJ, Martin RJ. Inhaled corticosteroids reduce the progression of airflow limitation in chronic obstructive pulmonary disease: a metaanalysis. Thorax. 2003; 58(11):937-41. [PubMed: 14586043]

Vestbo 1999 . Vestbo J, Sorensen T, Lange P, Brix A, Torre P, Viskum K. Long-term effect of inhaled budesonide in mild and moderate chronic obstructive pulmonary disease: a randomised controlled trial. Lancet. 1999; 353(9167):1819-23. [PubMed: 10359405]

Visual Rx . Cates, CJ. Visual Rx 2.0. 2003. www.nntonline.net

Walters 2007 . Walters EH, Gibson PG, Lasserson TJ, Walters JAE. Long-acting beta2-agonists for chronic asthma in adults and children where background therapy contains varied or no inhaled corticosteroid. Cochrane Database of Systematic Reviews. 2007; (1) Art. No.: CD001385. DOI: 10.1002/14651858.CD001385.pub2.

Yang 2007 . Yang IA, Fong KM, Sim EHA, Black PN, Lasserson TJ. Inhaled corticosteroids for stable chronic obstructive pulmonary disease. Cochrane Database of Systematic Reviews. 2007; (2) Art. No.: CD002991. DOI: 10.1002/14651858.CD002991.pub2.

\section{References to other published versions of this review}

Nannini 2003 . Nannini L, Lasserson TJ, Poole P. Combined corticosteroid and long-acting betaagonist in one inhaler for chronic obstructive pulmonary disease. Cochrane Database of Systematic Reviews. 2003; (4) Art. No.: CD003794. DOI: 10.1002/14651858.CD003794.pub2.

Nannini 2004 . Nannini L, Cates CJ, Lasserson TJ, Poole P. Combined corticosteroid and long-acting beta-agonist in one inhaler for chronic obstructive pulmonary disease. Cochrane Database of Systematic Reviews. 2004; (3)

* Indicates the major publication for the study 


\section{PLAIN LANGUAGE SUMMARY}

Combination therapy with inhaled corticosteroids and long-acting beta-agonists can reduce exacerbations and improve quality of life in people with chronic obstructive pulmonary disease (COPD) when compared to placebo treatment

Combinations of two classes of medication in one inhaler have been developed to treat people with COPD. Two types of combined inhaler exist currently: budesonide/ formoterol (BDF - 'Symbicort'), and fluticasone/salmeterol (FPS - 'Advair' or 'Seretide'). The results of the studies showed that combined inhalers were effective and reduced the frequency of exacerbations compared with placebo medication to a level of three quarters of the previous rates. The patients included in these trials had on average 1-2 exacerbations per year which means that treatment with combination therapy would lead to a reduction of one exacerbation every two to four years in these individuals. Combination therapy led to a reduction in mortality over three years, and also led to improvements in lung function and symptoms. However, there was an increased risk of pneumonia associated with combined inhalers, and further monitoring of this outcome in future trials would provide valuable information for consumers and clinicians. Future research is required to show whether there is a difference between combination inhalers with different strengths of inhaled corticosteroids. 


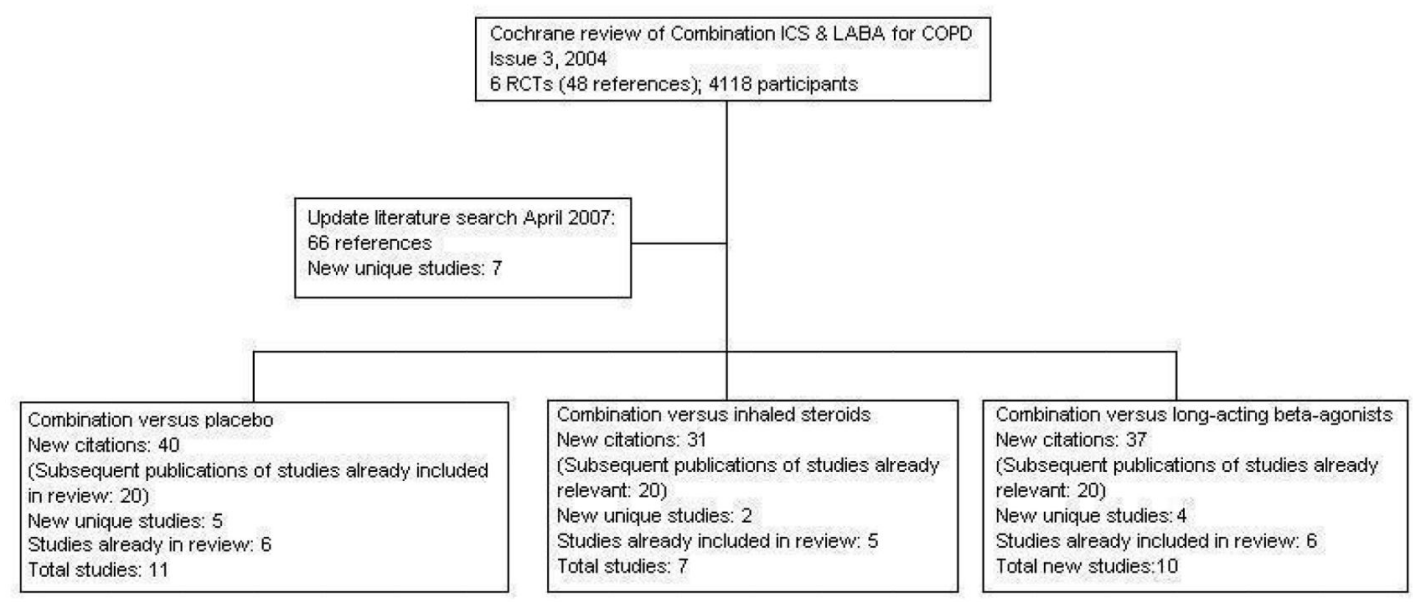

Figure 1. Flow chart to illustrate separation of review between three comparisons. Six RCTs met the original entry criteria of the review. All of these had a placebo and long-acting beta-agonist arm, and five assessed combination against steroids. Seven new studies with one or more control comparisons were identified: five had a placebo arm, three had a long-acting beta-agonist arm, and two had an inhaled steroid treatment arm 


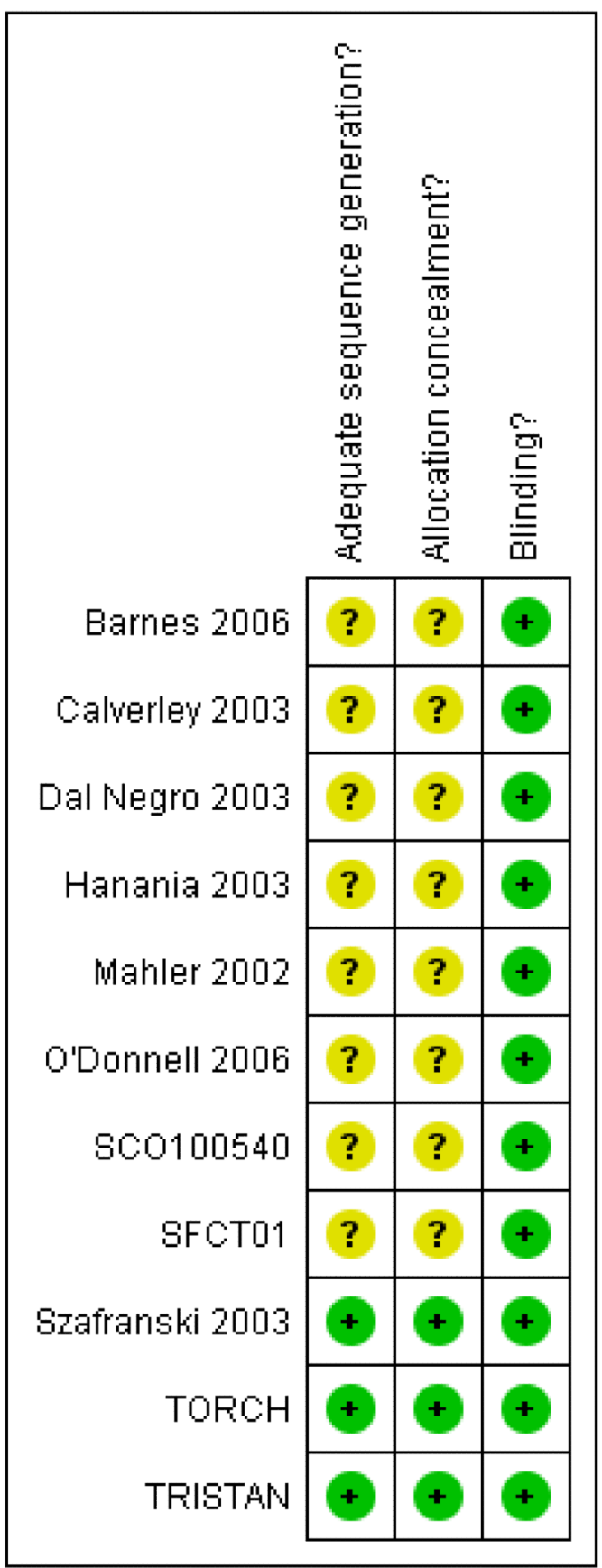

Figure 2. Methodological quality summary: review authors' judgements about each methodological quality item for each included study 


\begin{tabular}{|c|c|c|c|c|c|c|}
\hline \multicolumn{7}{|c|}{$\begin{array}{l}\text { Combined corticosteroid and long-acting beta-agonist in one inhaler for chronic obstructive pulmonary } \\
\text { disease }\end{array}$} \\
\hline \multicolumn{7}{|c|}{$\begin{array}{l}\text { Patient or population: patients with moderate and severe chronic obstructive pulmonary disease } \\
\text { Settings: outpatient } \\
\text { Intervention: corticosteroid and long-acting beta-agonist in one inhaler }{ }^{1} \\
\text { Comparision: no treatment }\end{array}$} \\
\hline \multirow[t]{2}{*}{ Outcomes } & \multicolumn{2}{|c|}{$\begin{array}{l}\text { Illustrative comparative risks* } \\
(95 \% \mathrm{CI})\end{array}$} & \multirow{2}{*}{$\begin{array}{l}\text { Relative } \\
\text { effect } \\
(95 \% \mathrm{Cl})\end{array}$} & \multirow{2}{*}{$\begin{array}{l}\text { No of } \\
\text { Participants } \\
\text { (studies) }\end{array}$} & \multirow{2}{*}{$\begin{array}{l}\text { Quality of } \\
\text { the } \\
\text { evidence } \\
\text { (GRADE) }\end{array}$} & \multirow[t]{2}{*}{ Comments } \\
\hline & $\begin{array}{l}\text { Assumed risk } \\
\text { no treatment }\end{array}$ & $\begin{array}{l}\text { Corresponding risk } \\
\text { combined inhaler }\end{array}$ & & & & \\
\hline $\begin{array}{l}\text { Exacerbation } \\
\text { rate } \\
\text { (follow-up: } 3 \\
\text { years) }\end{array}$ & $\begin{array}{l}\text { The mean } \\
\text { exacerbation rate } \\
\text { in the control } \\
\text { groups was } \\
3 \text { exacerbations } \\
\text { in } 3 \text { years }\end{array}$ & $\begin{array}{l}\text { The mean } \\
\text { exacerbation rate in } \\
\text { the intervention } \\
\text { groups was } \\
2 \text { exacerbations in } \\
3 \text { years }\end{array}$ & & $\begin{array}{l}4226 \\
(5)\end{array}$ & $\begin{array}{l}\oplus \oplus \oplus \mathrm{O} \\
\text { moderate }^{3}\end{array}$ & $\begin{array}{l}\text { Rate Ratio } 0.74 \\
(0.69,0.79)\end{array}$ \\
\hline Hospitalisations & See comment & See comment & Not estim & & $\begin{array}{l}\text { See } \\
\text { comment }\end{array}$ & $\begin{array}{l}\text { Limited data for } \\
\text { hospitalisations was } \\
\text { presented in the trials. }\end{array}$ \\
\hline \multirow{2}{*}{$\begin{array}{l}\text { Mortality } \\
\text { (follow-up: } 3 \\
\text { years) }\end{array}$} & \multicolumn{2}{|c|}{ Medium risk population ${ }^{2}$} & \multirow{2}{*}{$\begin{array}{l}\text { OR } 0.79 \\
(0.65 \text { to } \\
0.96)\end{array}$} & \multirow{2}{*}{$\begin{array}{l}5752 \\
(7)\end{array}$} & \multirow{2}{*}{$\begin{array}{l}\oplus \oplus \oplus \oplus \\
\text { high }\end{array}$} & \\
\hline & 15 per 100 & $\begin{array}{l}12 \text { per } 100 \\
(10 \text { to } 14)\end{array}$ & & & & \\
\hline $\begin{array}{l}\text { Quality of Life } \\
\text { St. George's } \\
\text { Respiratory } \\
\text { Questionnaire. } \\
\text { Scale from: } \\
0 \text { to } 100 . \\
\text { (follow-up: } 3 \\
\text { years) }\end{array}$ & $\begin{array}{l}\text { The mean } \\
\text { quality of life in } \\
\text { the control } \\
\text { groups was } \\
48 \text { points }\end{array}$ & $\begin{array}{l}\text { The mean Quality of } \\
\text { Life in the } \\
\text { intervention groups } \\
\text { was } \\
2.90 \text { lower } \\
\text { (3.61 to } 2.18 \text { lower) }\end{array}$ & & $\begin{array}{l}3346 \\
(4)\end{array}$ & $\begin{array}{l}\oplus \oplus \oplus \oplus \\
\text { high }\end{array}$ & $\begin{array}{l}\text { Difference did not } \\
\text { reach a patient } \\
\text { important } \\
\text { improvement of } 4 \\
\text { points. }\end{array}$ \\
\hline \multirow{2}{*}{$\begin{array}{l}\text { Pneumonia } \\
\text { (follow-up: } 3 \\
\text { years) }\end{array}$} & \multicolumn{2}{|c|}{ Medium risk population ${ }^{2}$} & \multirow{2}{*}{$\begin{array}{l}\text { OR } 1.83 \\
(1.51 \text { to } \\
2.21)\end{array}$} & \multirow{2}{*}{$\begin{array}{l}5739 \\
(8)\end{array}$} & \multirow{2}{*}{$\begin{array}{l}\oplus \oplus \oplus \oplus \\
\text { high }\end{array}$} & \\
\hline & 12 per 100 & $\begin{array}{l}20 \text { per } 100 \\
17 \text { to } 23 \text { ) }\end{array}$ & & & & \\
\hline \multirow{2}{*}{$\begin{array}{l}\text { Any adverse } \\
\text { events } \\
\text { (follow-up: } 3 \\
\text { years) }\end{array}$} & \multicolumn{2}{|c|}{ Medium risk population ${ }^{2}$} & \multirow{2}{*}{$\begin{array}{l}\text { OR } 1.10 \\
(0.96 \text { to } \\
1.27)\end{array}$} & \multirow{2}{*}{$\begin{array}{l}5493 \\
(8)\end{array}$} & \multirow{2}{*}{$\begin{array}{l}\oplus \oplus \oplus \oplus \\
\text { high }\end{array}$} & \multirow{2}{*}{$\begin{array}{l}\text { Data from } \\
\text { fluticasone/salmeterol } \\
\text { studies. }\end{array}$} \\
\hline & 90 per 100 & $\begin{array}{l}1 \text { per } 100 \\
90 \text { to } 92)\end{array}$ & & & & \\
\hline \multicolumn{7}{|c|}{$\begin{array}{l}\text { The basis for the assumed risk (e.g. th } \\
\text { corresponding risk (and its } 95 \% \text { confide } \\
\text { relative effect of the intervention (and its }\end{array}$} \\
\hline \multicolumn{7}{|c|}{ Cl: Confidence interval; OR: Odds ratio; } \\
\hline \multicolumn{7}{|c|}{$\begin{array}{l}\text { GRADE Working Group grades of evidence } \\
\text { High quality: Further research is very unlikely to change our confidence in the estimate of effect. } \\
\text { Moderate quality: Further research is likely to have an important impact on our confidence in the estimate of effect and may } \\
\text { change the estimate. } \\
\text { Low quality: Further research is very likely to have an important impact on our confidence in the estimate of effect and is } \\
\text { likely to change the estimate. } \\
\text { Very low quality: We are very uncertain about the estimate. }\end{array}$} \\
\hline \multicolumn{7}{|c|}{$\begin{array}{l}\text { Both long-acting beta-agonists and inhaled corticosteroids can be used in combination for the treatment of chronic } \\
\text { obstructive pulmonary disease. Of the } 11 \text { included studies, two evaluated fluticasone/salmeterol at } 250 \mathrm{mcg} / 50 \mathrm{mcg} \text { twice } \\
\text { daily and seven at } 500 \mathrm{mcg} / 50 \mathrm{mcg} \text { twice daily; and two evaluated budesonide/formoterol at } 320 \mathrm{mcg} / 9 \mathrm{mcg} \text { twice daily. All } \\
\text { studies permitted the use of inhaled short-acting beta-agonists (SABA) on demand. } \\
{ }^{2} \text { Assumed risk based on TORCH trial. } \\
{ }^{3} \text { Withdrawal of participants with severe frequent exacerbations may have biased results. }\end{array}$} \\
\hline
\end{tabular}

Figure 3. Summary of findings table for exacerbations, mortality, quality of life, pneumonia and adverse events 


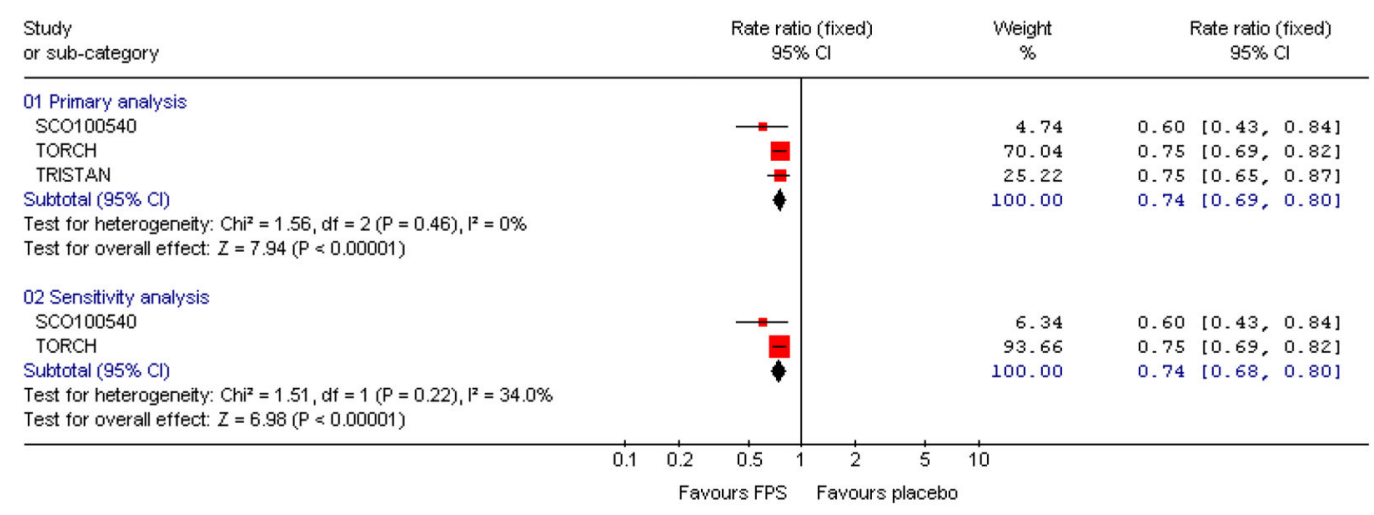

Figure 4. Forest plot of pooled rate ratios for combination fluticasone and salmeterol and placebo, with the summary estimate for TRISTAN included (primary analysis) and removed (sensitivity analysis) 


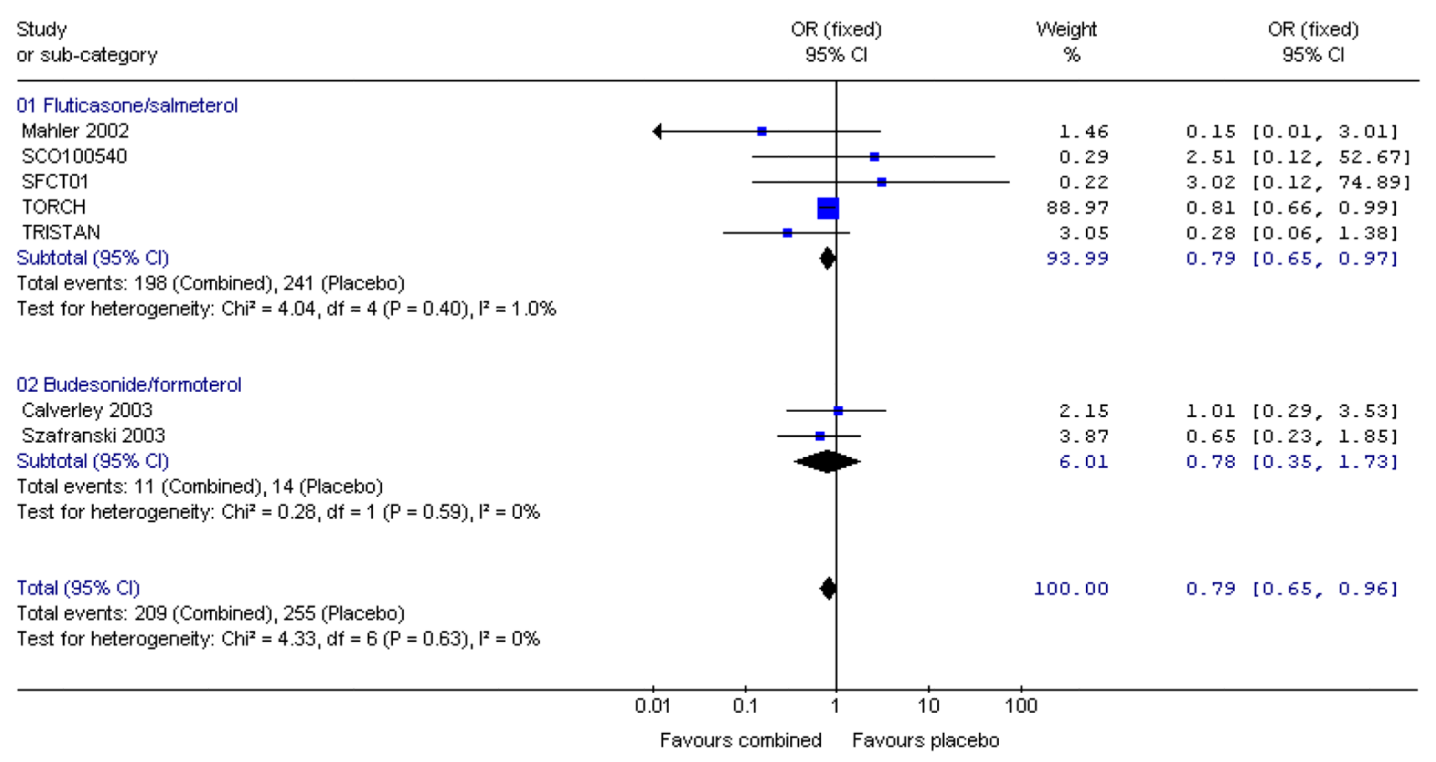

Figure 5. Pooled effect estimate on mortality for all combined inhalers versus placebo 


\section{Table 1}

Search history

\begin{tabular}{ll}
\hline Version & Detail \\
\hline 1st published version - Issue 4, & References identified: 34 \\
2003 (All years to April 2002) & References retrieved: 7 \\
& Studies excluded 3 (Cazzola 2000; Chapman 2002; Soriano 2002) \\
& Studies identified from supplementary searching: 4 (Dal Negro 2003; Hanania 2003 - both included; \\
& Cazzola 2002a; Cazzola 2004 - both excluded). \\
& Studies included: 4 \\
\hline 2nd published version - Issue 3, & References identified: 12 \\
2004 (April 2003-April 2004) & References retrieved: 3 (2 papers full publication of a previously included or cited studies study (Dal \\
& Negro 2003; Hanania 2003). \\
& Hand searching identified two further references to the COSMIC 2003 study. \\
& Studies identified from supplementary searching: 1 (TRISTAN 2003) \\
& New studies included: 2 \\
& Total studies included: 6 \\
\hline 3rd published version - Issue 3, & References identified: 52 \\
2005 (April 2004-April 2005) & References retrieved: 46 (references to studies already included/excluded/ongoing: 24) \\
& New unique studies identified: 10 (ongoing studies: 2) \\
& New studies included: 0 \\
& Total studies included: 6 \\
\hline 4th published version - April 2005 - & References identified: 66 \\
April 2007 & References retrieved: 27 (references to studies already included/ excluded/ongoing:) \\
& New unique studies identified: 8 (ongoing studies: 0) \\
& New studies included: 7 \\
& Total studies included: 13 \\
\hline
\end{tabular}




\section{Table 2}

Rates and NNT of mortality

\begin{tabular}{llll}
\hline Study ID & Study duration & Placebo rate (\%) & NNT \\
\hline TORCH & 156 weeks & 15.2 & $42(24$ to 387$)$ \\
\hline SCO100540 & 24 weeks & 0 & NA \\
\hline SFCT01 & 52 weeks & 0 & NA \\
\hline TRISTAN & 52 weeks & 1.94 & $292(169$ to 2628) \\
\hline Mahler 2002 & 24 weeks & 1.66 & $293(176$ to 1540$)$ \\
\hline Calverley 2003 & 52 weeks & 1.95 & $249(149$ to 1307$)$ \\
\hline Szafranski 2003 & 52 weeks & 4.5 & $110(66$ to 581$)$ \\
\hline
\end{tabular}


Table 3

Rates and NNT of pneumonia

\begin{tabular}{llll}
\hline Study ID & Study duration & Placebo rate (\%) & NNT(h) \\
\hline Hanania 2003 & 24 weeks & 0 & NA \\
\hline Mahler 2002 & 24 weeks & 0 & NA \\
\hline Barnes 2006 & 13 weeks & 0 & NA \\
\hline SCO100540 & 24 weeks & 0 & NA \\
\hline O'Donnell 2006 & 8 weeks & 1.56 & 83 \\
\hline TORCH & 156 weeks & 12.3 & 13 \\
\hline TRISTAN & 52 weeks & 0.83 & 153 \\
\hline Calverley 2003 & 52 weeks & 3.6 & 36 \\
\hline
\end{tabular}

